\title{
Projection-Reduction Approach to Optical Conductivities for an Electron-Phonon System and Their Diagram Representation
}

\author{
Nam Lyong Kang ${ }^{1}$ and Sang Don $\mathrm{Choi}^{2}$ \\ ${ }^{1}$ Department of Applied Nanoscience, Pusan National University, Miryang 627-706, Republic of Korea \\ ${ }^{2}$ Department of Physics, Kyungpook National University, Daegu 702-701, Republic of Korea \\ Correspondence should be addressed to Sang Don Choi; sdchoi@knu.ac.kr
}

Received 7 October 2013; Accepted 16 January 2014; Published 7 April 2014

Academic Editors: D. L. Nika and T. Yanagisawa

Copyright (C) 2014 N. L. Kang and S. D. Choi. This is an open access article distributed under the Creative Commons Attribution License, which permits unrestricted use, distribution, and reproduction in any medium, provided the original work is properly cited.

Utilizing state-dependent projection operators and the Kang-Choi reduction identities, we derive the linear, first, and secondorder nonlinear optical conductivities for an electron system interacting with phonons. The lineshape functions included in the conductivity tensors satisfy "the population criterion" saying that the Fermi distribution functions for electrons and Planck distribution functions for phonons should be combined in multiplicative forms. The results also contain energy denominator factors enforcing the energy conservation as well as interaction factors describing electron-phonon interaction properly. Therefore, the phonon absorption and emission processes as well as photon absorption and emission processes in all electron transition processes can be presented in an organized manner and the results can be represented in diagrams that can model the quantum dynamics of electrons in a solid.

\section{Introduction}

Studies of the optical transitions for electron systems interacting with a background are useful for examining the electronic properties of solids because the absorption lineshapes are sensitive to the type of scattering mechanisms affecting the carrier behavior. One of the approaches to this problem is the projection method [1-17], which is generally based on the response theory. The projection techniques can be divided into two kinds: single-electron projection techniques [1-9] and many-electron projection techniques [10-17].

A proper many-body theory should satisfy the so-called "many-body criteria," one of the most difficult being "the population criterion," meaning that the Fermi distribution functions for electrons and the Planck distribution functions for phonons should be combined in multiplicative forms because the electrons and phonons belong to different categories in quantum-statistical physics. The population factors satisfying the population criterion should be combined in a physically acceptable manner with the energy denominator factor to maintain energy conservation and with the interaction factor to interpret the occurrence of local fluctuations due to electron-phonon interactions in such a way that the transition can take place via implicit states. Theories based on the single-electron projection technique starting from the Kubo formalism have failed to meet this criterion.

The many-electron projection techniques are classified into two categories; the first being state-independent or current-dependent [10-13] and the second being statedependent [14-17]. The conductivity tensors [10,11] produced using the isolation and projection operators, which are stateindependent projection techniques, have limited applications for the following reasons. (a) They do not satisfy the population criterion because the two distribution functions are simply added (or subtracted), such as $N_{q}+1 / 2 \mp 1 / 2 \pm f_{\alpha}$, where $N_{q}$ and $f_{\alpha}$ are the Planck distribution function for a phonon with wave vector $q$ and the Fermi distribution function for an electron with energy $E_{\alpha}$, respectively. (b) They do not satisfy the "projection criterion," so they are applicable only to cases where the spacing between the energy levels is constant, such as cyclotron resonance phenomena; that is, they are unsuitable for a system with nonuniform energy separation, such as a quantum square well (see the statements below (51)).

Since Franken et al. [18] pioneered the field of nonlinear optical phenomena by the experimental work on optical wave 
mixing, it has attracted considerable research attention [19, 20]. Most studies focused on the electronic structures and the electromagnetic properties of media with increasing the available laser power [20-33], but there were some problems. Shen [22] did not have explicit forms of the damping terms (relaxation rates) appearing in the conductivity. Suzuki and Ashikawa [28] reported the explicit forms of the damping term but did not satisfy the population criterion.

The present authors introduced an identity relation (the Kang-Choi reduction identity (KCRI) of the first kind) to derive the lineshape formula for a cyclotron transition [12]. The population factors appearing in this formula satisfy the population criterion (see [12, equation (27)]). This shows that correct use of the KCRI can satisfy the population criterion. Later, the identity was generalized to cover the higher-order nonlinear optical conductivities [13-16]. The second kind (see [13, equation (4.18)]) was used along with current-dependent projectors, which is called the state-independent projectors, to derive the first-order nonlinear optical conductivity [13]. This satisfies the population criterion but has limited applications because it does not satisfy the projection criterion. The corrected extended version of the nonlinear optical conductivity formalism, including the second-order nonlinear optical conductivity, was presented recently by applying the projection-reduction (PR) method by combining the generalized state-dependent projection operator (SDPO) and the KCRI of the 3rd kind (see [14, equation (2.5)]). The axes of the generalized projectors are the creation-annihilation operator pairs (see [14, equation (6.4)]), which makes the formalism applicable to general electron systems; that is, it satisfies the projection criterion.

The aim of this study is to generalize the KCRI to cover higher-order nonlinear optical conductivities and show how physically acceptable forms of the optical conductivities can be obtained by PR method. In addition, a method is introduced to represent the optical conductivity formulae using diagrams, through which a physical intuition to the quantum dynamics of the electrons in a solid can be achieved.

\section{Hamiltonian and Density Operator}

To derive the optical conductivities, the Hamiltonian is defined and the density operator is introduced.

2.1. Hamiltonian. We consider the total Hamiltonian, $H_{\text {tot }}(t)$, for a system of electrons interacting with phonons as follows:

$$
H_{\text {tot }}(t)=H_{\text {eq }}+H_{\text {int }}(t) \text {, }
$$

where the time-independent part of the system in thermodynamic equilibrium, $H_{\mathrm{eq}}$, is given by

$$
\begin{aligned}
H_{\mathrm{eq}}= & H_{d}+V=H_{e}+H_{p}+V \\
= & \sum_{\alpha} E_{\alpha} a_{\alpha}^{+} a_{\alpha}+\sum_{q} \hbar \omega_{q} b_{q}^{+} b_{q} \\
& +\sum_{q} \sum_{\alpha, \beta} C_{\alpha \beta}(q) a_{\alpha}^{+} a_{\beta}\left(b_{q}+b_{-q}^{+}\right) .
\end{aligned}
$$

Here, $H_{e}, H_{p}$, and $V$ are the electron, phonon, and electronphonon interaction Hamiltonians, respectively, $a_{\alpha}^{+}\left(a_{\alpha}\right)$ is the creation (annihilation) operator for an electron in the state $|\alpha\rangle$ with energy $E_{\alpha}, b_{q}\left(b_{q}^{+}\right)$is that for a phonon in the state $|q\rangle$ with energy $\hbar \omega_{q},|q\rangle \equiv|\mathbf{q}, s\rangle$, $\mathbf{q}$ is the phonon wave vector, $s$ is the polarization index, and $C_{\alpha \beta}(q)$ is the electron-phonon interaction matrix element defined as

$$
C_{\alpha \beta}(q)=V_{q}\langle\alpha|\exp (i \mathbf{q} \cdot \mathbf{r})| \beta\rangle,
$$

where $V_{q}$ is the coupling factor that depends on the mode of phonons and $\mathbf{r}$ is the electron position vector.

In (1), when a linearly polarized time-dependent electric field with angular frequency $\omega, \mathbf{E}(t)=\mathbf{E}[\exp (i \omega t)+$ c.c], is applied, the following time-dependent interaction Hamiltonian is considered:

$$
H_{\text {int }}(t)=e \sum_{l=x, y, z, \gamma_{1}, \gamma_{1}^{\prime}}\left(r_{l}\right)_{\gamma_{1} \gamma_{1}^{\prime}} a_{\gamma_{1}}^{+} a_{\gamma_{1}^{\prime}} E_{l} \exp (i \omega t)+\text { c.c., }
$$

where $r_{x}, r_{y}$, and $r_{z}$ denote the $x, y$, and $z$ components, respectively, of the electron position vector, $(X)_{\alpha \beta} \equiv\langle\alpha|X| \beta\rangle$ for an arbitrary operator $X$, and c.c. means the complex conjugate.

2.2. Density Operator. To determine the general-order nonlinear optical conductivity, we assume that when the timedependent electric field is applied to the system, the total density operator can be split into two parts as follows:

$$
\rho_{\text {tot }}(t)=\rho_{\text {eq }}+\rho_{\text {int }}(t),
$$

where $\rho_{\text {eq }} \equiv \rho\left(H_{\text {eq }}\right)$ is the density operator for a system in thermal equilibrium and $\rho_{\text {int }}(t)$ is the perturbed term by the time-dependent external field. Using the Liouville equation

$$
i \hbar \frac{\partial \rho_{\mathrm{tot}}(t)}{\partial t}=\left[H_{\mathrm{tot}}(t), \rho_{\mathrm{tot}}(t)\right]=L_{\mathrm{tot}}(t) \rho_{\mathrm{tot}}(t)
$$

we can obtain

$$
i \hbar \frac{\partial \rho_{\mathrm{int}}(t)}{\partial t}=\left[H_{\mathrm{eq}}, \rho_{\mathrm{int}}(t)\right]+\left[H_{\mathrm{int}}(t), \rho_{\mathrm{eq}}\right]+\left[H_{\mathrm{int}}, \rho_{\mathrm{int}}(t)\right] \text {. }
$$

Here, we have used the fact that $i \hbar\left(\partial \rho_{\mathrm{eq}} / \partial t\right)=\left[H_{\mathrm{eq}}, \rho_{\mathrm{eq}}\right]=0$ and $L_{\text {tot }}(t)$ is the Liouville operator corresponding to the total Hamiltonian defined as $L_{\text {tot }}(t) X \equiv H_{\text {tot }}(t) X-X H_{\text {tot }}(t)$ for an arbitrary operator, $X . L_{\text {tot }}(t)$ can be split into two parts for calculation convenience: $L_{\text {tot }}(t)=L_{\text {eq }}+L_{\text {int }}(t)$ where $L_{\text {eq }}$ and $L_{\text {int }}(t)$, respectively, correspond to $H_{\text {eq }}$ and $H_{\text {int }}(t)$.

To obtain $\rho_{\text {int }}(t)$, we define the density operator in the Dirac picture as

$$
\rho_{\text {int }}^{D}(t) \equiv \exp \left(\frac{i H_{\mathrm{eq}} t}{\hbar}\right) \rho_{\text {int }}(t) \exp \left(-\frac{i H_{\mathrm{eq}} t}{\hbar}\right) .
$$

By differentiating (8) and considering (7),

$$
\begin{aligned}
i \hbar \frac{\partial \rho_{\text {int }}^{D}(t)}{\partial t}= & \exp \left(\frac{i L_{\mathrm{eq}} t}{\hbar}\right) L_{\text {int }}(t) \rho_{\mathrm{eq}} \\
& +\exp \left(\frac{i L_{\mathrm{eq}} t}{\hbar}\right) L_{\text {int }}(t) \rho_{\text {int }}(t),
\end{aligned}
$$


where we have used $\exp \left(i H_{\mathrm{eq}} t / \hbar\right) X \exp \left(-i H_{\mathrm{eq}} t / \hbar\right)=$ $\exp \left(i L_{\mathrm{eq}} t / \hbar\right) X$. Integrating (9) from $t=-\infty$ to $t=t$ subject to the initial condition $\rho_{\text {int }}^{D}(-\infty)=0$, a solution to this equation can be obtained:

$$
\begin{aligned}
i \hbar \rho_{\mathrm{int}}^{D}(t)= & \int_{-\infty}^{t} d u \exp \left(\frac{i L_{\mathrm{eq}} u}{\hbar}\right)\left\{L_{\mathrm{int}}(u) \rho_{\mathrm{eq}}\right\} \\
& +\int_{-\infty}^{t} d u \exp \left(\frac{i L_{\mathrm{eq}} u}{\hbar}\right)\left\{L_{\mathrm{int}}(u) \rho_{\mathrm{int}}(u)\right\} .
\end{aligned}
$$

By inserting (8) into (10) and replacing $t-u$ by $t_{1}$,

$$
\begin{aligned}
\rho_{\text {int }}(t)= & \frac{1}{i \hbar} \int_{0}^{\infty} d t_{1} \exp \left(-\frac{i L_{\text {eq }} t_{1}}{\hbar}\right)\left\{L_{\text {int }}\left(t-t_{1}\right) \rho_{\text {eq }}\right\} \\
& +\frac{1}{i \hbar} \int_{0}^{\infty} d t_{1} \exp \left(-\frac{i L_{\text {eq }} t_{1}}{\hbar}\right) \\
& \times\left\{L_{\text {int }}\left(t-t_{1}\right) \rho_{\text {int }}\left(t-t_{1}\right)\right\} .
\end{aligned}
$$

Finally, the following can be obtained by iteration:

$$
\begin{aligned}
\rho_{\text {int }}(t)= & \sum_{n=1}^{\infty} \frac{1}{(i \hbar)^{n}} \int_{0}^{\infty} d t_{1} \int_{0}^{\infty} d t_{2} \cdots \int_{0}^{\infty} d t_{n} \exp \left(-\frac{i L_{\mathrm{eq}} t_{1}}{\hbar}\right) \\
& \times L_{\mathrm{int}}\left(t-t_{1}\right) \exp \left(-\frac{i L_{\mathrm{eq}} t_{2}}{\hbar}\right) L_{\mathrm{int}}\left(t-t_{1}-t_{2}\right) \\
& \times \cdots \times \exp \left(-\frac{i L_{\mathrm{eq}} t_{n}}{\hbar}\right) L_{\mathrm{int}}\left(t-t_{1}-\cdots-t_{n}\right) \rho_{\mathrm{eq}} \\
\equiv & \rho^{(1)}(t)+\rho^{(2)}(t)+\cdots+\rho^{(n)}(t),
\end{aligned}
$$

where $\rho^{(n)}(t)$ involves $L_{\text {int }}(t)$ 's $n$ times.

Using (12), the ensemble average of the $k$ th component of the many-electron current density operator $J$ can be determined as follows:

$$
\left\langle J_{k}\right\rangle_{\mathrm{ens}}=\sum_{n=1}^{\infty}\left\langle J_{k}^{(n)}\right\rangle_{\mathrm{ens}}=\sum_{n=1}^{\infty} T_{R}\left\{\rho^{(n)}(t) J_{k}\right\},
$$

where $k=x, y, z$ and $T_{R}$ means the many-body trace. Equation (13) will be used in the following sections to calculate the linear and nonlinear optical conductivities.

\section{Optical Conductivity}

Using (12) and (13), the linear, first-, and second-order nonlinear optical conductivities are introduced.

3.1. Linear Optical Conductivity. The following can be obtained by inserting $\rho^{(1)}(t)$ in (12) into $\left\langle J_{k}^{(1)}\right\rangle_{\text {ens }}=$ $T_{R}\left\{J_{k} \rho^{(1)}(t)\right\}$ and considering (4):

$$
\begin{aligned}
\left\langle J_{k}^{(1)}\right\rangle_{\mathrm{ens}}= & \frac{e}{i \hbar} \sum_{l=x, y, z} \sum_{\gamma_{1}, \gamma_{1}^{\prime}}\left(r_{l}\right)_{\gamma_{1} \gamma_{1}^{\prime}} \\
& \times \int_{0}^{\infty} d t_{1} T_{R}\left\{\left(e^{i t_{1} L_{\mathrm{eq}} / \hbar} J_{k}\right)\left[a_{\gamma_{1}}^{+} a_{\gamma_{1}^{\prime}}, \rho_{\mathrm{eq}}\right]\right\} E_{l} e^{i \omega\left(t-t_{1}\right)},
\end{aligned}
$$

where we have used the cyclic property of trace, $T_{R}(A B C)=$ $T_{R}(B C A)$, and $\bar{\omega} \equiv \omega-i a\left(a \rightarrow 0^{+}\right)$. Using $T_{R}\{A[B, C]\}=$ $T_{R}\{C[A, B]\}$, (14) can be written as follows:

$$
\left\langle J_{k}^{(1)}\right\rangle_{\mathrm{ens}}=\sum_{l} \sigma_{k l}(\omega) E_{l}(\omega)
$$

Here, $E_{l}(\omega) \equiv E_{l} e^{i \omega t}$ and the linear optical conductivity is defined as follows:

$$
\sigma_{k l}(\omega)=-e \sum_{\gamma_{1}, \gamma_{1}^{\prime}}\left(r_{l}\right)_{\gamma_{1} \gamma_{1}^{\prime}} T_{R}\left\{\rho_{\mathrm{eq}}\left[G(\bar{\omega}) J_{k}, a_{\gamma_{1}}^{+} a_{\gamma_{1}^{\prime}}\right]\right\}
$$

where

$$
G(\bar{\omega}) \equiv\left(\hbar \bar{\omega}-L_{\text {eq }}\right)^{-1}
$$

3.2. First-Order Nonlinear Optical Conductivity. Considering $\rho^{(2)}(t)$ in (12) and $T_{R}(A B C)=T_{R}(B C A),\left\langle J_{k}^{(2)}\right\rangle_{\text {ens }}=$ $T_{R}\left\{J_{k} \rho^{(2)}(t)\right\}$ becomes

$$
\begin{aligned}
\left\langle J_{k}^{(2)}\right\rangle_{\text {ens }}= & \frac{1}{(i \hbar)^{2}} \int_{0}^{\infty} d t_{1} \int_{0}^{\infty} d t_{2} \\
& \times T_{R}\left\{A_{L}\left[H_{\text {int }}\left(t-t_{1}\right), e^{-i H_{\mathrm{eq}} t_{2} / \hbar} G_{\rho} e^{i H_{\mathrm{eq}} t_{2} / \hbar}\right]\right\},
\end{aligned}
$$

where $A_{L} \equiv e^{i L_{\mathrm{eq}} t_{1} / \hbar} J_{k}$ and $G_{\rho} \equiv L_{\text {int }}\left(t-t_{1}-t_{2}\right) \rho_{\mathrm{eq}}$. Using $T_{R}\{A[B, C]\}=T_{R}\{C[A, B]\}$, (18) becomes

$$
\begin{aligned}
\left\langle J_{k}^{(2)}\right\rangle_{\mathrm{ens}}= & \frac{1}{(i \hbar)^{2}} \int_{0}^{\infty} d t_{1} \int_{0}^{\infty} d t_{2} T_{R} \\
& \times\left\{\rho _ { \mathrm { eq } } \left[e^{i L_{\mathrm{eq}} t_{2} / \hbar}\left[e^{i L_{\mathrm{eq}} t_{1} / \hbar} J_{k}, H_{\mathrm{int}}\left(t-t_{1}\right)\right],\right.\right. \\
& \left.\left.H_{\mathrm{int}}\left(t-t_{1}-t_{2}\right)\right]\right\} .
\end{aligned}
$$

Inserting (4) into (19),

$$
\begin{aligned}
& \left\langle J_{k}^{(2)}\right\rangle_{\mathrm{ens}} \\
& =\left(\frac{e}{i \hbar}\right)^{2} \sum_{l, m} \sum_{\gamma_{1}, \gamma_{1}^{\prime}} \sum_{\gamma_{2}, \gamma_{2}^{\prime}}\left(r_{l}\right)_{\gamma_{1} \gamma_{1}^{\prime}}\left(r_{m}\right)_{\gamma_{2} \gamma_{2}^{\prime}} E_{l}\left(\omega_{1}\right) E_{m}\left(\omega_{2}\right) \\
& \quad \times \int_{0}^{\infty} d t_{1} \int_{0}^{\infty} d t_{2} T_{R}\left\{\rho _ { \mathrm { eq } } \left[e^{i t_{2}\left(L_{\mathrm{eq}}-\hbar \bar{\omega}_{1}\right) / \hbar}\right.\right. \\
& \left.\left.\quad \times\left[e^{i t_{1}\left(L_{\mathrm{eq}}-\hbar \bar{\omega}_{12}\right) / \hbar} J_{k}, a_{\gamma_{1}}^{+} a_{\gamma_{1}^{\prime}}\right], a_{\gamma_{2}}^{+} a_{\gamma_{2}^{\prime}}\right]\right\} \\
& =\sum_{k, l} \sigma_{k l m}\left(\omega_{1}, \omega_{2}\right) E_{l}\left(\omega_{2}\right) E_{m}\left(\omega_{1}\right),
\end{aligned}
$$


where $\omega_{12} \equiv \omega_{1}+\omega_{2}$ and the first-order nonlinear optical conductivity is defined as follows:

$$
\begin{aligned}
& \sigma_{k l m}\left(\omega_{1}, \omega_{2}\right) \\
& =e^{2} \sum_{\gamma_{1}, \gamma_{1}^{\prime}} \sum_{\gamma_{2}, \gamma_{2}^{\prime}}\left(r_{l}\right)_{\gamma_{1} \gamma_{1}^{\prime}}\left(r_{m}\right)_{\gamma_{2} \gamma_{2}^{\prime}} \\
& \quad \times T_{R}\left\{\rho_{\mathrm{eq}}\left[G\left(\bar{\omega}_{1}\right)\left[G\left(\bar{\omega}_{12}\right) J_{k}, a_{\gamma_{1}}^{+} a_{\gamma_{1}^{\prime}}\right], a_{\gamma_{2}}^{+} a_{\gamma_{2}^{\prime}}\right]\right\} .
\end{aligned}
$$

3.3. Second-Order Nonlinear Optical Conductivity. Inserting $\rho^{(3)}(t)$ in (12) into $\left\langle J_{k}^{(3)}\right\rangle_{\text {ens }}=T_{R}\left\{J_{k} \rho^{(3)}(t)\right\}$,

$$
\begin{aligned}
& \left\langle J_{k}^{(3)}\right\rangle_{\mathrm{ens}} \\
& =\frac{1}{(i \hbar)^{3}} \int_{0}^{\infty} d t_{1} \int_{0}^{\infty} d t_{2} \int_{0}^{\infty} d t_{3} T_{R} \\
& \quad \times\left\{D_{F}\left[H_{\mathrm{int}}\left(t-t_{1}-t_{2}\right), e^{-i H_{\mathrm{eq}} t_{3} / \hbar} T_{\rho} e^{i H_{\mathrm{eq}} t_{3} / \hbar}\right]\right\},
\end{aligned}
$$

where $D_{L} \equiv e^{i L_{\mathrm{eq}} t_{2} / \hbar}\left[A_{L}, H_{\mathrm{int}}\left(t-t_{1}\right)\right], A_{L} \equiv e^{i L_{\mathrm{eq}} t_{1} / \hbar} J_{k}$, and $T_{\rho} \equiv L_{\text {int }}\left(t-t_{1}-t_{2}-t_{3}\right) \rho_{\mathrm{eq}}$. Considering $T_{R}\{A[B, C]\}=$ $T_{R}\{[A, B] C\}$ and inserting $G_{\rho}$ into (22),

$$
\begin{aligned}
\left\langle J_{k}^{(3)}\right\rangle_{\text {ens }}= & \frac{1}{(i \hbar)^{3}} \int_{0}^{\infty} d t_{1} \int_{0}^{\infty} d t_{2} \int_{0}^{\infty} d t_{3} T_{R} \\
& \times\left\{\rho_{\mathrm{eq}}\left[F_{L}, H_{\mathrm{int}}\left(t-t_{1}-t_{2}-t_{3}\right)\right]\right\},
\end{aligned}
$$

where $F_{L} \equiv e^{i L_{\mathrm{eq}} t_{3} / \hbar}\left[D_{L}, H_{\text {int }}\left(t-t_{1}-t_{2}\right)\right]$. Therefore, inserting $F_{L}, D_{L}$, and $A_{L}$ into (23) results in

$$
\begin{aligned}
& \left\langle J_{k}^{(3)}\right\rangle_{\mathrm{ens}} \\
& =\frac{1}{(i \hbar)^{3}} \int_{0}^{\infty} d t_{1} \int_{0}^{\infty} d t_{2} \int_{0}^{\infty} d t_{3} \\
& \quad \times T_{R}\left\{\rho _ { \mathrm { eq } } \left[e ^ { i L _ { \mathrm { eq } } t _ { 3 } / \hbar } \left[e^{i L_{\mathrm{eq}} t_{2} / \hbar}\left[e^{i L_{\mathrm{eq}} t_{1} / \hbar} J_{k}, H_{\mathrm{int}}\left(t-t_{1}\right)\right],\right.\right.\right. \\
& \left.\left.\left.\quad H_{\mathrm{int}}\left(t-t_{1}-t_{2}\right)\right] H_{\mathrm{int}}\left(t-t_{1}-t_{2}-t_{3}\right)\right]\right\} .
\end{aligned}
$$

Finally, considering (4), we obtain

$$
\begin{aligned}
& \left\langle J_{k}^{(3)}\right\rangle_{\mathrm{ens}} \\
& =\left(\frac{e}{i \hbar}\right)^{3} \sum_{l, m, n} \sum_{\gamma_{1}, \gamma_{1}^{\prime} \gamma_{2}, \gamma_{2}^{\prime}} \sum_{\gamma_{3}, \gamma_{3}^{\prime}}\left(r_{l}\right)_{\gamma_{1} \gamma_{1}^{\prime}}\left(r_{m}\right)_{\gamma_{2} \gamma_{2}^{\prime}}\left(r_{n}\right)_{\gamma_{3} \gamma_{3}^{\prime}} \\
& \quad \times \int_{0}^{\infty} d t_{1} \int_{0}^{\infty} d t_{2} \int_{0}^{\infty} d t_{3} T_{R}\left\{\rho _ { \mathrm { eq } } \left[e^{i t_{3}\left(L_{\mathrm{eq}}-\hbar \bar{\omega}_{1}\right) / \hbar}\right.\right. \\
& \quad \times\left[e^{i t_{2}\left(L_{\mathrm{eq}}-\hbar \bar{\omega}_{12}\right) / \hbar}\left[e^{i t_{1}\left(L_{\mathrm{eq}}-\hbar \bar{\omega}_{123}\right) / \hbar} J_{k}, a_{\gamma_{1}}^{+} a_{\gamma_{1}^{\prime}}\right],\right. \\
& \left.\left.\quad \times E_{l}\left(\omega_{1}\right) E_{m}\left(\omega_{2}\right) E_{n}\left(\omega_{3}\right), a_{\gamma_{3}}^{+} a_{\gamma_{3}^{\prime}}\right]\right\} \\
& =\sum_{l, m, n} \sigma_{k l m n}\left(\omega_{1}, \omega_{2}, \omega_{3}\right) E_{l}\left(\omega_{1}\right) E_{m}\left(\omega_{2}\right) E_{n}\left(\omega_{3}\right),
\end{aligned}
$$

where $\omega_{123} \equiv \omega_{1}+\omega_{2}+\omega_{3}$ and the second-order nonlinear optical conductivity is defined as

$$
\begin{aligned}
& \sigma_{k l m n}\left(\omega_{1}, \omega_{2}, \omega_{3}\right) \\
& =-e^{3} \sum_{\gamma_{1}, \gamma_{1}^{\prime}} \sum_{\gamma_{2}, \gamma_{2}^{\prime}} \sum_{\gamma_{3}, \gamma_{3}^{\prime}}\left(r_{l}\right)_{\gamma_{1} \gamma_{1}^{\prime}}\left(r_{m}\right)_{\gamma_{2} \beta_{2}^{\prime}}\left(r_{n}\right)_{\gamma_{3} \gamma_{3}^{\prime}} \\
& \times T_{R}\left\{\rho _ { \mathrm { eq } } \left[G\left(\bar{\omega}_{1}\right)\right.\right. \\
& \left.\left.\times\left[G\left(\bar{\omega}_{12}\right)\left[G\left(\bar{\omega}_{123}\right) J_{k}, a_{\gamma_{1}}^{+} a_{\gamma_{1}^{\prime}}\right], a_{\gamma_{2}}^{+} a_{\gamma_{2}^{\prime}}\right], a_{\gamma_{3}}^{+} a_{\gamma_{3}^{\prime}}\right]\right\} .
\end{aligned}
$$

\section{Kang-Choi Reduction Identity}

The essential part of this paper is expansion of the optical conductivities (see (16), (21) and (26)) to easily solvable forms. For this purpose, this section introduces various forms of a useful identity, known as the Kang-Choi reduction identity (KCRI).

4.1. KCRI of First Kind (KCRI-I). For arbitrary operators, $X$ and $A_{1}$, we consider

$$
\begin{aligned}
T_{R} & \left\{\rho_{\mathrm{eq}}\left[L_{\mathrm{eq}} X, A_{1}\right]\right\} \\
& =T_{R}\left\{\rho_{\mathrm{eq}}\left(H_{\mathrm{eq}} X A_{1}-X H_{\mathrm{eq}} A_{1}-A_{1} H_{\mathrm{eq}} X+A_{1} X H_{\mathrm{eq}}\right)\right\} .
\end{aligned}
$$

Considering $\left[H_{\mathrm{eq}}, \rho_{\mathrm{eq}}\right]=0$ and the trace property, $T_{R}(A B C)=T_{R}(B C A)$, for the first and fourth terms, the KCRI-I of rank 1 [(KCRI-I $)_{1}$ ] can be obtained as follows:

$$
T_{R}\left\{\rho_{\mathrm{eq}}\left[L_{\mathrm{eq}} X, A_{1}\right]\right\}=-T_{R}\left\{\rho_{\mathrm{eq}}\left[X, L_{\mathrm{eq}} A_{1}\right]\right\}
$$

which is useful because $L$ and $X$ are separated. Similarly, for arbitrary operators, $X, A_{1}$, and $A_{2}$,

$$
\begin{aligned}
T_{R}\left\{\rho_{\mathrm{eq}}\left[\left[L_{\mathrm{eq}} X, A_{1}\right], A_{2}\right]\right\} & \\
=T_{R}\left\{\rho_{\mathrm{eq}}(\right. & H_{\mathrm{eq}} X A_{1} A_{2}-A_{2} H_{\mathrm{eq}} X A_{1} \\
& -A_{1} H_{\mathrm{eq}} X A_{2}+A_{2} A_{1} H_{\mathrm{eq}} X \\
& -X H_{\mathrm{eq}} A_{1} A_{2}+A_{2} X H_{\mathrm{eq}} A_{1} \\
& \left.\left.+A_{1} X H_{\mathrm{eq}} A_{2}-A_{2} A_{1} X H_{\mathrm{eq}}\right)\right\} .
\end{aligned}
$$

Considering $\left[H_{\text {eq }}, \rho_{\text {eq }}\right]=0$ and $T_{R}(A B C)=T_{R}(B C A)$ for the first and eighth terms and adding

$$
\begin{aligned}
T_{R}\left\{\rho_{\mathrm{eq}}(\right. & -X A_{1} H_{\mathrm{eq}} A_{2}+H_{\mathrm{eq}} A_{2} X A_{1} \\
& +H_{\mathrm{eq}} A_{1} X A_{2}-A_{2} H_{\mathrm{eq}} A_{1} X \\
& +X A_{1} H_{\mathrm{eq}} A_{2}-H_{\mathrm{eq}} A_{2} X A_{1} \\
& \left.\left.-H_{\mathrm{eq}} A_{1} X A_{2}+A_{2} H_{\mathrm{eq}} A_{1} X\right)\right\}
\end{aligned}
$$


which is null, to the right-hand side of (29), the KCRI-I of rank $2\left[(\mathrm{KCRI}-\mathrm{I})_{2}\right]$ can be obtained as follows:

$$
\begin{aligned}
T_{R}\left\{\rho_{\mathrm{eq}}\left[\left[L_{\mathrm{eq}} X, A_{1}\right], A_{2}\right]\right\}= & -T_{R}\left\{\rho_{\mathrm{eq}}\left[\left[X, L_{\mathrm{eq}} A_{1}\right], A_{2}\right]\right\} \\
& -T_{R}\left\{\rho_{\mathrm{eq}}\left[\left[X, A_{1}\right], L_{\mathrm{eq}} A_{2}\right]\right\} .
\end{aligned}
$$

Therefore, the KCRI-I of rank $k\left[(\mathrm{KCRI}-\mathrm{I})_{k}\right]$ can be inferred as follows:

$$
\begin{aligned}
T_{R}\left\{\rho_{\mathrm{eq}}\left[\cdots\left[\left[L_{\mathrm{eq}} X, A_{1}\right], A_{2}\right], \ldots, A_{k}\right]\right\} \\
=-T_{R}\left\{\rho _ { \mathrm { eq } } \left(\left[\cdots\left[\left[X, L_{\mathrm{eq}} A_{1}\right], A_{2}\right], \ldots, A_{k}\right]\right.\right. \\
+\left[\cdots\left[\left[X, A_{1}\right], L_{\mathrm{eq}} A_{2}\right], \ldots, A_{k}\right] \\
\left.\left.+\cdots+\left[\cdots\left[\left[X, A_{1}\right], A_{2}\right], \ldots, L_{\mathrm{eq}} A_{k}\right]\right)\right\},
\end{aligned}
$$

where $k=1,2,3, \ldots$. The present form given by (32) is the general case. The proof for $k=3$ is as follows. The left-hand side (lhs) of (32) for $k=3$ can be expanded as follows:

$$
\begin{aligned}
\text { lhs } \equiv T_{R}\left\{\rho_{\mathrm{eq}}[[\right. & {\left.\left.\left.\left[L_{\mathrm{eq}} X, A_{1}\right], A_{2}\right], A_{3}\right]\right\} } \\
=T_{R}\left\{\rho_{\mathrm{eq}}[\right. & X A_{1} A_{2} A_{3} H_{\mathrm{eq}}-A_{3} H_{\mathrm{eq}} X A_{1} A_{2} \\
& -A_{2} H_{\mathrm{eq}} X A_{1} A_{3}+A_{3} A_{2} H_{\mathrm{eq}} X A_{1} \\
& -A_{1} H_{\mathrm{eq}} X A_{2} A_{3}+A_{3} A_{1} H_{\mathrm{eq}} X A_{2} \\
+ & \left.A_{2} A_{1} H_{\mathrm{eq}} X A_{3}-A_{3} A_{2} A_{1} H_{\mathrm{eq}} X\right) \\
+ & -X H_{\mathrm{eq}} A_{1} A_{2} A_{3}+A_{3} X H_{\mathrm{eq}} A_{1} A_{2} \\
& +A_{2} X H_{\mathrm{eq}} A_{1} A_{3}-A_{3} A_{2} X H_{\mathrm{eq}} A_{1} \\
& +A_{1} X H_{\mathrm{eq}} A_{2} A_{3}-A_{3} A_{1} X H_{\mathrm{eq}} A_{2} \\
& \left.\left.\left.-A_{2} A_{1} X H_{\mathrm{eq}} A_{3}+H_{\mathrm{eq}} A_{3} A_{2} A_{1} X\right)\right]\right\}
\end{aligned}
$$

which is the same as the right-hand side (rhs) of (32) for $k=$ 3.

4.2. KCRI of Second Kind (KCRI-II). The KCRI of the second kind (KCRI-II) is given by the following:

$$
\begin{aligned}
T_{R}\left\{\rho_{\mathrm{eq}}\left[\left(\hbar \bar{\omega}-L_{\mathrm{eq}}\right)^{-1}\left[L_{\mathrm{eq}} X, A_{1}\right], A_{2}\right]\right\} \\
=-T_{R}\left\{\rho_{\mathrm{eq}}\left[\left(\hbar \bar{\omega}-L_{\mathrm{eq}}\right)^{-1}\left[X, L_{\mathrm{eq}} A_{1}\right], A_{2}\right]\right. \\
\left.+\rho_{\mathrm{eq}}\left[\left(\hbar \bar{\omega}-L_{\mathrm{eq}}\right)^{-1}\left[X, A_{1}\right], L_{\mathrm{eq}} A_{2}\right]\right\},
\end{aligned}
$$

which will be used for derivation of the first-order nonlinear optical conductivity formula. Equation (34) can be proven by applying the identity

$$
(A-B)^{-1}=A^{-1}+A^{-1} B(A-B)^{-1}
$$

on $\left(\hbar \bar{\omega}-L_{\text {eq }}\right)^{-1}$; that is, the lhs of (34) can be changed into

$$
\text { lhs }=\sum_{n=0}^{\infty}\left(\frac{1}{\hbar \bar{\omega}}\right)^{n+1} T_{R}\left\{\rho_{\mathrm{eq}}\left[L_{\mathrm{eq}}^{n}\left[L_{\mathrm{eq}} X, A_{1}\right], A_{2}\right]\right\} \text {. }
$$

Applying (KCRI-I $)_{1}$ with $X \equiv\left[L_{\mathrm{eq}} X, A_{1}\right]$ and $A_{1} \equiv A_{2}$ gives the following:

$$
\begin{aligned}
\text { lhs } & =\sum_{n=0}^{\infty}\left(\frac{1}{\hbar \bar{\omega}}\right)^{n+1} T_{R}\left\{\rho_{\mathrm{eq}}\left[\left[L_{\mathrm{eq}} X, A_{1}\right],\left(-L_{\mathrm{eq}}\right)^{n} A_{2}\right]\right\} \\
& =T_{R}\left\{\rho_{\mathrm{eq}}\left[\left[L_{\mathrm{eq}} X, A_{1}\right],\left(\bar{\omega}+L_{\mathrm{eq}}\right)^{-1} A_{2}\right]\right\} .
\end{aligned}
$$

Therefore, applying (KCRI-I) ${ }_{2}$ results in

$$
\begin{aligned}
\text { lhs }=-T_{R}\left\{\rho_{\mathrm{eq}}[\right. & {\left.\left[X, L_{\mathrm{eq}} A_{1}\right],\left(\hbar \bar{\omega}+L_{\mathrm{eq}}\right)^{-1} A_{2}\right] } \\
& \left.+\rho_{\mathrm{eq}}\left[\left[X, A_{1}\right],\left(\hbar \bar{\omega}+L_{\mathrm{eq}}\right)^{-1} L_{\mathrm{eq}} A_{2}\right]\right\}
\end{aligned}
$$

which is reduced to the rhs of (34) by applying (35) in a reverse manner.

4.3. A Lemma Useful for Derivation of the KC Expansion Relation. A lemma useful to derive the general form of KCRI is introduced. For an analytic function of $L_{\mathrm{eq}}, f\left(L_{\mathrm{eq}}\right)$,

$$
\begin{gathered}
T_{R}\left\{\rho_{\mathrm{eq}}\left[\cdots\left[f\left(L_{\mathrm{eq}}\right)\left[L_{\mathrm{eq}} X, A_{1}\right], A_{2}\right], \ldots, A_{k}\right]\right\} \\
=-T_{R}\left\{\rho _ { \mathrm { eq } } \left(\left[\cdots\left[f\left(L_{\mathrm{eq}}\right)\left[X, L_{\mathrm{eq}} A_{1}\right], A_{2}\right], \ldots, A_{k}\right]\right.\right. \\
+\left[\cdots\left[f\left(L_{\mathrm{eq}}\right)\left[X, A_{1}\right], L_{\mathrm{eq}} A_{2}\right], \ldots, A_{k}\right] \\
+\cdots+\left[\cdots\left[f\left(L_{\mathrm{eq}}\right)\left[X, A_{1}\right], A_{2}\right], \ldots,\right. \\
\left.\left.\left.L_{\mathrm{eq}} A_{k}\right]\right)\right\}
\end{gathered}
$$

for $k=1,2,3, \ldots$. It suffices to prove the case of $f\left(L_{\text {eq }}\right)=L_{\text {eq }}$, because $f\left(L_{\text {eq }}\right)$ can be expanded in a Taylor series. The case for $k=3$ is dealt with for illustration. Using (KCRI-I) $)_{2}$,

$$
\begin{aligned}
T_{R}\left\{\rho_{\mathrm{eq}}[\right. & {\left.\left.\left[L_{\mathrm{eq}}\left[L_{\mathrm{eq}} X, A_{1}\right], A_{2}\right], A_{3}\right]\right\} } \\
=-T_{R}\{ & \left\{\rho_{\mathrm{eq}}\left[\left[\left[L_{\mathrm{eq}} X, A_{1}\right], L_{\mathrm{eq}} A_{2}\right], A_{3}\right]\right. \\
& \left.\quad+\rho_{\mathrm{eq}}\left[\left[\left[L_{\mathrm{eq}} X, A_{1}\right], A_{2}\right], L_{\mathrm{eq}} A_{3}\right]\right\} .
\end{aligned}
$$

Applying (KCRI-I) $)_{3}$, the rhs of (40) becomes

$$
\begin{aligned}
\text { rhs }=T_{R}\left\{\rho_{\mathrm{eq}}(\right. & {\left[\left[\left[X, L_{\mathrm{eq}} A_{1}\right], L_{\mathrm{eq}} A_{2}\right], A_{3}\right] } \\
& +\left[\left[\left[X, A_{1}\right], L_{\mathrm{eq}}^{2} A_{2}\right], A_{3}\right] \\
& +\left[\left[\left[X, A_{1}\right], L_{\mathrm{eq}} A_{2}\right], L_{\mathrm{eq}} A_{3}\right] \\
& +\left[\left[\left[X, L_{\mathrm{eq}} A_{1}\right], A_{2}\right], L_{\mathrm{eq}} A_{3}\right] \\
& +\left[\left[\left[X, A_{1}\right], L_{\mathrm{eq}} A_{2}\right], L_{\mathrm{eq}} A_{3}\right] \\
& \left.\left.+\left[\left[\left[X, A_{1}\right], A_{2}\right], L_{\mathrm{eq}}^{2} A_{3}\right]\right)\right\} .
\end{aligned}
$$


Therefore, applying (KCRI-I $)_{2}$ in a reverse manner results in

rhs of (40)

$$
\begin{aligned}
=-T_{R}\left\{\rho_{\mathrm{eq}}\right. & \left(\left[\left[L_{\mathrm{eq}}\left[X, L_{\mathrm{eq}} A_{1}\right], A_{2}\right], A_{3}\right]\right. \\
& +\left[\left[L_{\mathrm{eq}}\left[X, A_{1}\right], L_{\mathrm{eq}} A_{2}\right], A_{3}\right] \\
& \left.\left.+\left[\left[L_{\mathrm{eq}}\left[X, A_{1}\right], A_{2}\right], L_{\mathrm{eq}} A_{3}\right]\right)\right\}
\end{aligned}
$$

= rhs of (39) for $k=3, f\left(L_{\mathrm{eq}}\right)=L_{\mathrm{eq}}$

completing the proof for $k=3$. The cases for $f\left(L_{\mathrm{eq}}\right)=L_{\mathrm{eq}}^{2}=$ $L_{\text {eq }} \cdot L_{\text {eq }}$ and $f\left(L_{\text {eq }}\right)=L_{\text {eq }}^{3}=L_{\text {eq }}^{2} \cdot L_{\text {eq }}$ can be proven by an iterative operation of (40). Using this lemma, the calculations needed to prove the KCRI for $n \geq 3$ are reduced.

4.4. KCRI of Third Kind (KCRI-III). The KCRI of the 3rd kind (KCRI-III) can be expressed as

$$
\begin{aligned}
T_{R}\left\{\rho_{\mathrm{eq}}[\right. & \left(\hbar \bar{\omega}_{1}-L_{\mathrm{eq}}\right)^{-1} \\
\times & {\left.\left.\left[\left(\hbar \bar{\omega}_{12}-L_{\mathrm{eq}}\right)^{-1}\left[L_{\mathrm{eq}} X, A_{1}\right], A_{2}\right], A_{3}\right]\right\} } \\
=-T_{R}\left\{\rho _ { \mathrm { eq } } \left(\left[\left(\hbar \bar{\omega}_{1}-L_{\mathrm{eq}}\right)^{-1}\right.\right.\right. & \left.\times\left[\left(\hbar \bar{\omega}_{12}-L_{\mathrm{eq}}\right)^{-1}\left[X, L_{\mathrm{eq}} A_{1}\right], A_{2}\right], A_{3}\right] \\
+ & {\left[\left(\hbar \bar{\omega}_{1}-L_{\mathrm{eq}}\right)^{-1}\right.} \\
& \left.\times\left[\left(\hbar \bar{\omega}_{12}-L_{\mathrm{eq}}\right)^{-1}\left[X, A_{1}\right], L_{\mathrm{eq}} A_{2}\right], A_{3}\right] \\
+ & {\left[\left(\hbar \bar{\omega}_{1}-L_{\mathrm{eq}}\right)^{-1}\right.} \\
& \left.\left.\left.\times\left[\left(\hbar \bar{\omega}_{12}-L_{\mathrm{eq}}\right)^{-1}\left[X, A_{1}\right], A_{2}\right], L_{\mathrm{eq}} A_{3}\right]\right)\right\}
\end{aligned}
$$

The proof can be obtained as follows. From (37), the lhs of (43) becomes

$$
\begin{gathered}
\text { lhs }=T_{R}\left\{\rho _ { \mathrm { eq } } \left[\left[\left(\hbar \bar{\omega}_{12}-L_{\mathrm{eq}}\right)^{-1}\left[L_{\mathrm{eq}} X, A_{1}\right], A_{2}\right],\right.\right. \\
\left.\left.\left(\hbar \bar{\omega}_{1}+L_{\mathrm{eq}}\right)^{-1} A_{3}\right]\right\} \\
=-T_{R}\left\{\rho _ { \mathrm { eq } } \left(\left[\left[\left(\hbar \bar{\omega}_{12}-L_{\mathrm{eq}}\right)^{-1}\left[X, L_{\mathrm{eq}} A_{1}\right], A_{2}\right],\right.\right.\right. \\
\left.\left(\hbar \bar{\omega}_{1}+L_{\mathrm{eq}}\right)^{-1} A_{3}\right] \\
+\left[\left[\left(\hbar \bar{\omega}_{12}-L_{\mathrm{eq}}\right)^{-1}\left[X, A_{1}\right], L_{\mathrm{eq}} A_{2}\right],\right. \\
\left.\left(\hbar \bar{\omega}_{1}+L_{\mathrm{eq}}\right)^{-1} A_{3}\right]
\end{gathered}
$$

$$
\begin{gathered}
+\left[\left[\left(\hbar \bar{\omega}_{12}-L_{\mathrm{eq}}\right)^{-1}\left[X, A_{1}\right], A_{2}\right]\right. \\
\left.\left.\left.\left(\hbar \bar{\omega}_{1}+L_{\mathrm{eq}}\right)^{-1} L_{\mathrm{eq}} A_{3}\right]\right)\right\},
\end{gathered}
$$

where the KC expansion relation (see (39)) was applied to the last step. Therefore, applying (37) in a reverse manner results in the right-hand side of (43). KCRI-III (see (43)) is needed in deriving the second-order nonlinear optical conductivity formula.

\section{State-Dependent Projection Operators}

In this section, we calculate the linear, first-, and second-order optical conductivities using the PR method.

5.1. Linear Optical Conductivity. Using the many-electron current density operator $J_{k}$, which is written in terms of the single-electron current operator, $j_{k}=(i e \hbar / m) \partial / \partial r_{k}(k=$ $x, y, x)$, as

$$
J_{k}=\sum_{\alpha, \beta}\left(j_{k}\right)_{\alpha \beta} a_{\alpha}^{+} a_{\beta}
$$

the linear optical conductivity (16) becomes

$$
\sigma_{k l}(\omega)=-e \sum_{\alpha, \beta} \sum_{\gamma_{1}, \gamma_{1}^{\prime}}\left(j_{k}\right)_{\alpha \beta}\left(r_{l}\right)_{\gamma_{1} \gamma_{1}^{\prime}} R_{1}(\omega),
$$

where

$$
R_{1}(\omega) \equiv T_{R}\left\{\rho_{\mathrm{eq}}\left[G(\bar{\omega}) a_{\alpha}^{+} a_{\beta}, a_{\gamma_{1}}^{+} a_{\gamma_{1}^{\prime}}\right]\right\} .
$$

To calculate $R_{1}(\omega)$, the first state-dependent projection operators for an arbitrary operator $X$ are introduced as follows:

$$
P_{1} X=\frac{\langle X\rangle_{\gamma_{1}}}{\left\langle a_{\alpha}^{+} a_{\beta}\right\rangle_{\gamma_{1}}} a_{\alpha}^{+} a_{\beta}, \quad Q_{1}=1-P_{1},
$$

where

$$
\langle X\rangle_{\gamma_{1}} \equiv T_{R}\left\{\rho_{\mathrm{eq}}\left[X, a_{\gamma_{1}}^{+} a_{\gamma_{1}^{\prime}}\right]\right\} .
$$

Applying the identity, $1=P_{1}+Q_{1}$, to the right-hand side of the Liouville operator in (47) as $L_{\mathrm{eq}}=L_{\mathrm{eq}}\left(P_{1}+Q_{1}\right)$ and using (35) give

$$
\begin{aligned}
\frac{1}{\hbar \bar{\omega}-L_{\mathrm{eq}}} a_{\alpha}^{+} a_{\beta}= & \frac{1}{\hbar \bar{\omega}-L_{\mathrm{eq}} Q_{1}} a_{\alpha}^{+} a_{\beta} \\
& +\frac{1}{\hbar \bar{\omega}-L_{\mathrm{eq}} Q_{1}} L_{\mathrm{eq}} P_{1} \frac{1}{\hbar \bar{\omega}-L_{\mathrm{eq}} Q_{1}} a_{\alpha}^{+} a_{\beta}
\end{aligned}
$$

which can be rearranged as

$$
\begin{aligned}
\frac{1}{\hbar \bar{\omega}-L_{\mathrm{eq}}} a_{\alpha}^{+} a_{\beta}= & \frac{1}{\hbar \bar{\omega}} a_{\alpha}^{+} a_{\beta}+\frac{E_{\alpha \beta}}{\hbar \bar{\omega}} a_{\alpha}^{+} a_{\beta} \frac{R_{1}(\omega)}{\left\langle a_{\alpha}^{+} a_{\beta}\right\rangle_{\gamma_{1}}} \\
& +\frac{1}{\hbar \bar{\omega}-L_{\mathrm{eq}} Q_{1}} L_{\nu} a_{\alpha}^{+} a_{\beta} \frac{R_{1}(\omega)}{\left\langle a_{\alpha}^{+} a_{\beta}\right\rangle_{\gamma_{1}}} .
\end{aligned}
$$


Here, we have used $Q_{1} a_{\alpha}^{+} a_{\beta}=0$ and $L_{d} a_{\alpha}^{+} a_{\beta}=E_{\alpha \beta} a_{\alpha}^{+} a_{\beta}$ where $E_{\alpha \beta} \equiv E_{\alpha}-E_{\beta}, L_{\mathrm{eq}}=L_{d}+L_{v}$, and $L_{d}$ and $L_{v}$ are the Liouville operators corresponding to $H_{d}$ and $V$, respectively.

Note that the projection operators given in (48) are statedependent; that is, $P_{1}$ projects an arbitrary operator $X$ onto the operator $a_{\alpha}^{+} a_{\beta}$, which depends on the states $\alpha$ and $\beta$. On the other hand, state-independent projection operators project $X$ into the state-independent current density operator, $J_{k}$. The state-independent projection operator method is applicable only to the case in which $L_{d} J_{k}=c$-number $\times J_{k}$ or $Q_{1} L_{d} J_{k}=0$. Cyclotron phenomenon belongs to this category because for the current operator, $J_{ \pm}=J_{x} \pm i J_{y}, L_{d} J_{+}=$ $\hbar \omega_{c} J_{+}$, where $\omega_{c}$ is the cyclotron frequency. This is called the "projection criterion." Equation (48) satisfies this criterion because $Q_{1} L_{d} a_{\alpha}^{+} a_{\beta}=0$.

Taking the ensemble average of (51) gives

$$
R_{1}(\omega)=\frac{\left\langle a_{\alpha}^{+} a_{\beta}\right\rangle_{\gamma_{1}}}{\hbar \bar{\omega}-E_{\alpha \beta}-S_{1}(\bar{\omega})} .
$$

Here

$$
S_{1}(\bar{\omega})\left\langle a_{\alpha}^{+} a_{\beta}\right\rangle_{\gamma_{1}} \equiv \hbar \bar{\omega}\left\langle\left(\hbar \bar{\omega}-L_{\mathrm{ew}} Q_{1}\right)^{-1} L_{v} a_{\alpha}^{+} a_{\beta}\right\rangle_{\gamma_{1}}
$$

which can be rearranged as

$$
\begin{aligned}
S_{1}(\bar{\omega}) & \left\langle a_{\alpha}^{+} a_{\beta}\right\rangle_{\gamma_{1}} \\
= & \left\langle L_{v} a_{\alpha}^{+} a_{\beta}\right\rangle_{\gamma_{1}} \\
& -T_{R}\left\{\rho_{\mathrm{eq}}\left[Q_{1}\left(\hbar \bar{\omega}-L_{\mathrm{eq}} Q_{0}\right)^{-1} L_{v} a_{\alpha}^{+} a_{\beta}, L_{\mathrm{eq}} a_{\gamma_{1}}^{+} a_{\gamma_{1}^{\prime}}\right]\right\},
\end{aligned}
$$

where we have used (35) and (KCRI-I) ${ }_{1}$ in the second term. To calculate (54) further, we make use of the following relation in the second term of (54):

$$
\begin{aligned}
T_{R}\left\{\rho_{\mathrm{eq}}\right. & {\left.\left[Q_{1} X, L_{\mathrm{eq}} a_{\gamma_{1}}^{+} a_{\gamma_{1}^{\prime}}\right]\right\} } \\
= & E_{\gamma_{1} \gamma_{1}^{\prime}} T_{R}\left\{\rho_{\mathrm{eq}}\left[X, a_{\gamma_{1}}^{+} a_{\gamma_{1}^{\prime}}\right]\right\}-E_{\gamma_{1} \gamma_{1}^{\prime}} T_{R}\left\{\rho_{\mathrm{eq}}\left[P_{1} X, a_{\gamma_{1}}^{+} a_{\gamma_{1}^{\prime}}\right]\right\} \\
& +T_{R}\left\{\rho_{\mathrm{eq}}\left[X, L_{v} a_{\gamma_{1}}^{+} a_{\gamma_{1}^{\prime}}\right]\right\}-T_{R}\left\{\rho_{\mathrm{eq}}\left[P_{1} X, L_{v} a_{\gamma_{1}}^{+} a_{\gamma_{1}^{\prime}}\right]\right\},
\end{aligned}
$$

where the first and second terms are canceled out. In this stage, $\rho\left(H_{\mathrm{eq}}\right) \approx \rho\left(H_{d}\right)$ is assumed and the terms up to the second order of $L_{v}$ are considered, assuming that the electron-phonon interaction is quite weak. Then, the first term in (54) and the fourth term in (55) are neglected because the ensemble averages of $b_{q}$ and $b_{q}^{+}$are zeros. Therefore,

$$
S_{1}(\bar{\omega})\left\langle a_{\alpha}^{+} a_{\beta}\right\rangle_{\gamma_{1}}=-T_{R}\left\{\rho_{\mathrm{eq}}\left[G_{d}(\bar{\omega}) L_{v} a_{\alpha}^{+} a_{\beta}, L_{v} a_{\gamma_{1}}^{+} a_{\gamma_{1}^{\prime}}\right]\right\},
$$

where

$$
G_{d}(\bar{\omega}) \equiv\left(\hbar \bar{\omega}-L_{d}\right)^{-1} .
$$

Equation (56) is an easily calculable form because it contains neither $L_{v}$ nor the projection operator in the denominator.
5.2. First-Order Nonlinear Optical Conductivity. By inserting (45) into (21), the first-order nonlinear optical conductivity becomes

$$
\begin{aligned}
& \sigma_{k l m}\left(\omega_{1}, \omega_{2}\right) \\
& =e^{2} \sum_{\alpha, \beta} \sum_{\gamma_{1}, \gamma_{1}^{\prime} \gamma_{2}, \gamma_{2}^{\prime}}\left(j_{k}\right)_{\alpha \beta}\left(r_{l}\right)_{\gamma_{1} \gamma_{1}^{\prime}}\left(r_{m}\right)_{\gamma_{2} \gamma_{2}^{\prime}} R_{2}\left(\omega_{1}, \omega_{2}\right),
\end{aligned}
$$

where

$$
\begin{aligned}
& R_{2}\left(\omega_{1}, \omega_{2}\right) \\
& \quad \equiv T_{R}\left\{\rho_{\mathrm{eq}}\left[G\left(\bar{\omega}_{1}\right)\left[G\left(\bar{\omega}_{12}\right) a_{\alpha}^{+} a_{\beta}, a_{\gamma_{1}}^{+} a_{\gamma_{1}^{\prime}}\right], a_{\gamma_{2}}^{+} a_{\gamma_{2}^{\prime}}\right]\right\},
\end{aligned}
$$

which can be calculated by defining the second statedependent projection operators for an arbitrary operator, $X$, as follows:

$$
P_{2} X=\frac{\langle X\rangle_{\gamma_{1} \gamma_{2}}}{\left\langle a_{\alpha}^{+} a_{\beta}\right\rangle_{\gamma_{1} \gamma_{2}}} a_{\alpha}^{+} a_{\beta}, \quad Q_{2}=1-P_{2},
$$

where

$$
\langle X\rangle_{\gamma_{1} \gamma_{2}} \equiv T_{R}\left\{\rho_{\mathrm{eq}}\left[G\left(\bar{\omega}_{1}\right)\left[X, a_{\gamma_{1}}^{+} a_{\gamma_{1}^{\prime}}\right], a_{\gamma_{2}}^{+} a_{\gamma_{2}^{\prime}}\right]\right\} .
$$

Applying the identity, $1=P_{2}+Q_{2}$, to the right-hand side of the Liouville operator in (59) as $\left(\hbar \bar{\omega}_{12}-L_{\text {eq }}\right)^{-1}=\left(\hbar \bar{\omega}_{12}-\right.$ $\left.L_{\mathrm{eq}} P_{2}-L_{\mathrm{eq}} Q_{2}\right)^{-1}$ and using the identity (35) give the following after a similar procedure as that used in (52):

$$
R_{2}\left(\omega_{1}, \omega_{2}\right)=\frac{\left\langle a_{\alpha}^{+} a_{\beta}\right\rangle_{\gamma_{1} \gamma_{2}}}{\hbar \bar{\omega}_{12}-E_{\alpha \beta}-S_{2}\left(\bar{\omega}_{1}, \bar{\omega}_{2}\right)} .
$$

Here

$$
S_{2}\left(\bar{\omega}_{1}, \bar{\omega}_{2}\right)\left\langle a_{\alpha}^{+} a_{\beta}\right\rangle_{\gamma_{1}, \gamma_{2}} \equiv D_{1}\left(\bar{\omega}_{1}\right)+D_{2}\left(\bar{\omega}_{1}, \bar{\omega}_{2}\right),
$$

where

$$
\begin{aligned}
D_{1}\left(\bar{\omega}_{1}\right) \equiv T_{R}\left\{\rho_{\mathrm{eq}}[\right. & \left.\left.G\left(\bar{\omega}_{1}\right)\left[L_{v} a_{\alpha}^{+} a_{\beta}, a_{\gamma_{1}}^{+} a_{\gamma_{1}^{\prime}}\right], a_{\gamma_{2}}^{+} a_{\gamma_{2}^{\prime}}\right]\right\}, \\
D_{2}\left(\bar{\omega}_{1}, \bar{\omega}_{2}\right) & \\
\equiv T_{R}\left\{\rho _ { \mathrm { eq } } \left[G\left(\bar{\omega}_{1}\right)[\right.\right. & L_{\mathrm{eq}} Q_{2}\left(\hbar \bar{\omega}_{12}-L_{\mathrm{eq}} Q_{2}\right)^{-1} \\
& \left.\left.\left.\times L_{v} a_{\alpha}^{+} a_{\beta}, a_{\gamma_{1}}^{+} a_{\gamma_{1}^{\prime}}\right], a_{\gamma_{2}}^{+} a_{\gamma_{2}^{\prime}}\right]\right\} .
\end{aligned}
$$

5.2.1. Calculation of $D_{1}\left(\bar{\omega}_{1}\right)$. Applying (35) to (64) with $A \equiv$ $\hbar \bar{\omega}_{1}$ and $B \equiv L_{\text {eq }}$ results in

$$
\begin{aligned}
D_{1}\left(\bar{\omega}_{1}\right)= & -\left(\frac{1}{\hbar \bar{\omega}_{1}}\right) T_{R} \\
& \times\left\{\rho_{\mathrm{eq}}\left[G\left(\bar{\omega}_{1}\right)\left[L_{v} a_{\alpha}^{+} a_{\beta}, a_{\gamma_{1}}^{+} a_{\gamma_{1}^{\prime}}\right], L_{\mathrm{eq}} a_{\gamma_{2}}^{+} a_{\gamma_{2}^{\prime}}\right]\right\}
\end{aligned}
$$


in the second-order approximation in $L_{v}$, where we have used the (KCRI-I $)_{1}$. Considering $L_{d} a_{\gamma_{2}}^{+} a_{\gamma_{2}^{\prime}}=E_{\gamma_{2} \gamma_{2}^{\prime}} a_{\gamma_{2}}^{+} a_{\gamma_{2}^{\prime}}$,

$$
D_{1}\left(\bar{\omega}_{1}\right)=-\frac{V_{11}\left(\hbar \bar{\omega}_{1}\right)}{\left(\hbar \bar{\omega}_{1}+E_{\gamma_{2} \gamma_{2}^{\prime}}\right)} \text {, }
$$

where

$$
V_{11}\left(\bar{\omega}_{1}\right) \equiv T_{R}\left\{\rho_{\mathrm{eq}}\left[G_{d}\left(\bar{\omega}_{1}\right)\left[L_{\nu} a_{\alpha}^{+} a_{\beta}, a_{\gamma_{1}}^{+} a_{\gamma_{1}^{\prime}}\right], L_{v} a_{\gamma_{2}}^{+} a_{\gamma_{2}^{\prime}}\right]\right\} .
$$

5.2.2. Calculation of $D_{2}\left(\bar{\omega}_{1}, \bar{\omega}_{2}\right)$. Applying KCRI-II to (65) results in

$$
\begin{aligned}
& D_{2}\left(\bar{\omega}_{1}, \bar{\omega}_{2}\right) \\
&=-T_{R}\left\{\rho _ { \mathrm { eq } } \left[G\left(\bar{\omega}_{1}\right)[\right.\right. Q_{2}\left(\hbar \bar{\omega}_{12}-L_{\mathrm{eq}} Q_{2}\right)^{-1} \\
&\left.\left.\left.\times L_{v} a_{\alpha}^{+} a_{\beta}, L_{\mathrm{eq}} a_{\gamma_{1}}^{+} a_{\gamma_{1}^{\prime}}\right], a_{\gamma_{2}}^{+} a_{\gamma_{2}^{\prime}}\right]\right\} \\
&-T_{R}\left\{\rho _ { \mathrm { eq } } \left[G\left(\bar{\omega}_{1}\right)[\right.\right. {\left[Q_{2}\left(\hbar \bar{\omega}_{12}-L_{\mathrm{eq}} Q_{2}\right)^{-1}\right.} \\
& \times\left.\left.\left.L_{v} a_{\alpha}^{+} a_{\beta}, a_{\gamma_{1}}^{+} a_{\gamma_{1}^{\prime}}\right], L_{\mathrm{eq}} a_{\gamma_{2}}^{+} a_{\gamma_{2}^{\prime}}\right]\right\} .
\end{aligned}
$$

Considering that

$$
\begin{aligned}
T_{R}\left\{\rho _ { \mathrm { eq } } \left[G\left(\bar{\omega}_{1}\right)[\right.\right. & \mathrm{Q}_{2}\left(\hbar \bar{\omega}_{12}-L_{\mathrm{eq}} \mathrm{Q}_{2}\right)^{-1} \\
& \left.\left.\left.\times L_{v} a_{\alpha}^{+} a_{\beta}, a_{\gamma_{1}}^{+} a_{\gamma_{1}^{\prime}}\right], a_{\gamma_{2}}^{+} a_{\gamma_{2}^{\prime}}\right]\right\}=0,
\end{aligned}
$$

we obtain, in the second-order approximation in $L_{v}$,

$$
D_{2}\left(\bar{\omega}_{1}, \bar{\omega}_{2}\right)=-\left(\hbar \bar{\omega}_{1}+E_{\gamma_{2} \gamma_{2}^{\prime}}\right)^{-1} V_{12}\left(\bar{\omega}_{1}, \bar{\omega}_{2}\right)-V_{13}\left(\bar{\omega}_{1}, \bar{\omega}_{2}\right),
$$

where we have used (37) in the first term of (69),

$$
\begin{aligned}
& V_{12}\left(\bar{\omega}_{1}, \bar{\omega}_{2}\right) \\
& \quad \equiv T_{R}\left\{\rho_{\mathrm{eq}}\left[\left[G_{d}\left(\bar{\omega}_{12}\right) L_{v} a_{\alpha}^{+} a_{\beta}, L_{v} a_{\gamma_{1}}^{+} a_{\gamma_{1}^{\prime}}\right], a_{\gamma_{2}}^{+} a_{\gamma_{2}^{\prime}}\right]\right\}, \\
& V_{13}\left(\bar{\omega}_{1}, \bar{\omega}_{2}\right) \\
& \quad \equiv T_{R}\left\{\rho_{\mathrm{eq}}\left[G_{d}\left(\bar{\omega}_{1}\right)\left[G_{d}\left(\bar{\omega}_{12}\right) L_{v} a_{\alpha}^{+} a_{\beta}, a_{\gamma_{1}}^{+} a_{\gamma_{1}^{\prime}}\right], L_{v} a_{\gamma_{2}}^{+} a_{\gamma_{2}^{\prime}}\right]\right\} .
\end{aligned}
$$

Therefore, inserting (67) and (71) into (63) results in

$$
\begin{aligned}
S_{2}\left(\bar{\omega}_{1}, \bar{\omega}_{2}\right)= & -\frac{1}{\bar{K}_{2}\left(\bar{\omega}_{1}\right)} \\
& \times\left[\frac{V_{11}\left(\bar{\omega}_{1}\right)+V_{12}\left(\bar{\omega}_{1}, \bar{\omega}_{2}\right)}{\hbar \bar{\omega}_{1}+E_{\gamma_{2} \gamma_{2}^{\prime}}}+V_{13}\left(\bar{\omega}_{1}, \bar{\omega}_{2}\right)\right]
\end{aligned}
$$

in the second-order approximation in $L_{v}$, where

$$
\bar{K}_{2}\left(\bar{\omega}_{1}\right) \equiv T_{R}\left\{\rho_{\mathrm{eq}}\left[G_{d}\left(\bar{\omega}_{1}\right)\left[a_{\alpha}^{+} a_{\beta}, a_{\gamma_{1}}^{+} a_{\gamma_{1}^{\prime}}\right], a_{\gamma_{2}}^{+} a_{\gamma_{2}^{\prime}}\right]\right\} .
$$

Note that $V_{i j}$ values in (74) are already approximated up to the second order of $L_{v}$, so $\left\langle a_{\alpha}^{+} a_{\beta}\right\rangle_{\gamma_{1} \gamma_{2}}$ is approximated to $\bar{K}_{2}\left(\bar{\omega}_{1}\right)$.

5.3. Second-Order Nonlinear Optical Conductivity. By applying (45) to (26), the second-order nonlinear optical conductivity becomes

$$
\begin{aligned}
& \sigma_{k l m n}\left(\omega_{1}, \omega_{2}, \omega_{3}\right) \\
& =-e^{3} \sum_{\alpha, \beta} \sum_{\gamma_{1}, \gamma_{1}^{\prime}} \sum_{\gamma_{2}, \gamma_{2}^{\prime}} \sum_{\gamma_{3}, \gamma_{3}^{\prime}}\left(j_{k}\right)_{\alpha \beta}\left(r_{l}\right)_{\gamma_{1} \gamma_{1}^{\prime}}\left(r_{m}\right)_{\gamma_{2} \gamma_{2}^{\prime}} \\
& \quad \times\left(r_{n}\right)_{\gamma_{3} \gamma_{3}^{\prime}} R_{3}\left(\omega_{1}, \omega_{2}, \omega_{3}\right),
\end{aligned}
$$

where

$$
\begin{aligned}
& R_{3}\left(\omega_{1}, \omega_{2}, \omega_{3}\right) \\
& \equiv T_{R}\left\{\rho _ { \mathrm { eq } } \left[G ( \overline { \omega } _ { 1 } ) \left[G\left(\bar{\omega}_{12}\right)\left[G\left(\bar{\omega}_{123}\right) a_{\alpha}^{+} a_{\beta}, a_{\gamma_{1}}^{+} a_{\gamma_{1}^{\prime}}\right],\right.\right.\right. \\
& \left.\left.\left.a_{\gamma_{2}}^{+} a_{\gamma_{2}^{\prime}}\right], a_{\gamma_{3}}^{+} a_{\gamma_{3}^{\prime}}\right]\right\} .
\end{aligned}
$$

To calculate (77), the third state-dependent projection operators for an arbitrary operator, $X$, are defined as follows:

$$
P_{3} X=\frac{\langle X\rangle_{\gamma_{1} \gamma_{2} \gamma_{3}}}{\left\langle a_{\alpha}^{+} a_{\beta}\right\rangle_{\gamma_{1} \gamma_{2} \gamma_{3}}} a_{\alpha}^{+} a_{\beta}, \quad Q_{3}=1-P_{3},
$$

where

$$
\begin{gathered}
\langle X\rangle_{\gamma_{1} \gamma_{2} \gamma_{3}} \equiv T_{R}\left\{\rho _ { \mathrm { eq } } \left[G ( \overline { \omega } _ { 1 } ) \left[G\left(\bar{\omega}_{12}\right)\left[X, a_{\gamma_{1}}^{+} a_{\gamma_{1}^{\prime}}\right],\right.\right.\right. \\
\left.\left.\left.a_{\gamma_{2}}^{+} a_{\gamma_{2}^{\prime}}\right], a_{\gamma_{3}}^{+} a_{\gamma_{3}^{\prime}}\right]\right\} .
\end{gathered}
$$

Applying the identity, $1=P_{3}+Q_{3}$, to the right-hand side of the Liouville operator in $(77)$ as $\left(\hbar \bar{\omega}_{123}-L_{\text {eq }}\right)^{-1}=$ $\left(\hbar \bar{\omega}_{123}-L_{\text {eq }} P_{3}-L_{\text {eq }} Q_{3}\right)^{-1}$ and using (35) result in the following after a similar procedure as that used in (52) and (62):

$$
R_{3}\left(\omega_{1}, \omega_{2}, \omega_{3}\right)=\frac{\left\langle a_{\alpha}^{+} a_{\beta}\right\rangle_{\gamma_{1} \gamma_{2} \gamma_{3}}}{\hbar \bar{\omega}_{123}-E_{\alpha \beta}-S_{3}\left(\bar{\omega}_{1}, \bar{\omega}_{2}, \bar{\omega}_{3}\right)} .
$$

Here

$$
\begin{aligned}
& S_{3}\left(\bar{\omega}_{1}, \bar{\omega}_{2}, \bar{\omega}_{3}\right)\left\langle a_{\alpha}^{+} a_{\beta}\right\rangle_{\gamma_{1}, \gamma_{2}, \gamma_{3}} \\
& \quad \equiv E_{1}\left(\bar{\omega}_{1}, \bar{\omega}_{2}\right)+E_{2}\left(\bar{\omega}_{1}, \bar{\omega}_{2}, \bar{\omega}_{3}\right),
\end{aligned}
$$

where

$$
\begin{aligned}
& E_{1}\left(\bar{\omega}_{1}, \bar{\omega}_{2}\right) \\
& \equiv T_{R}\left\{\rho _ { \mathrm { eq } } \left[G ( \overline { \omega } _ { 1 } ) \left[G\left(\bar{\omega}_{12}\right)\left[L_{v} a_{\alpha}^{+} a_{\beta}, a_{\gamma_{1}}^{+} a_{\gamma_{1}^{\prime}}\right],\right.\right.\right. \\
& \left.\left.\left.\quad a_{\gamma_{2}}^{+} a_{\gamma_{2}^{\prime}}\right], a_{\gamma_{3}}^{+} a_{\gamma_{3}^{\prime}}\right]\right\},
\end{aligned}
$$




$$
\begin{aligned}
& E_{2}\left(\bar{\omega}_{1}, \bar{\omega}_{2}, \bar{\omega}_{3}\right) \\
& \equiv T_{R}\left\{\rho _ { \mathrm { eq } } \left[G ( \overline { \omega } _ { 1 } ) \left[G\left(\bar{\omega}_{12}\right)\right.\right.\right. \\
& \times\left[L_{\mathrm{eq}} Q_{3}\left(\hbar \bar{\omega}_{123}-L_{\mathrm{eq}} Q_{3}\right)^{-1} L_{v} a_{\alpha}^{+} a_{\beta}, a_{\gamma_{1}}^{+} a_{\gamma_{1}^{\prime}}\right], \\
& \left.\left.\left.a_{\gamma_{2}}^{+} a_{\gamma_{2}^{\prime}}\right], a_{\gamma_{3}}^{+} a_{\gamma_{3}^{\prime}}\right]\right\} .
\end{aligned}
$$

5.3.1. Calculation of $E_{1}\left(\bar{\omega}_{1}, \bar{\omega}_{2}\right)$. Considering (35) with $A \equiv$ $\hbar \bar{\omega}_{12}$ and $B \equiv L_{\text {eq }},(82)$ can be expanded as follows:

$$
E_{1}\left(\bar{\omega}_{1}, \bar{\omega}_{2}\right) \hbar \bar{\omega}_{12}=E_{11}\left(\bar{\omega}_{1}\right)+E_{12}\left(\bar{\omega}_{1}, \omega_{2}\right),
$$

where

$$
\begin{gathered}
E_{11}\left(\bar{\omega}_{1}\right) \equiv T_{R}\left\{\rho _ { \mathrm { eq } } \left[G ( \overline { \omega } _ { 1 } ) \left[\left[L_{v} a_{\alpha}^{+} a_{\beta}, a_{\gamma_{1}}^{+} a_{\gamma_{1}^{\prime}}\right],\right.\right.\right. \\
\left.\left.\left.a_{\gamma_{2}}^{+} a_{\gamma_{2}^{\prime}}\right], a_{\gamma_{3}}^{+} a_{\gamma_{3}^{\prime}}\right]\right\}, \\
E_{12}\left(\bar{\omega}_{1}, \omega_{2}\right) \equiv T_{R}\left\{\rho _ { \mathrm { eq } } \left[G ( \overline { \omega } _ { 1 } ) \left[L _ { \mathrm { eq } } G ( \overline { \omega } _ { 1 2 } ) \left[L_{v} a_{\alpha}^{+} a_{\beta},\right.\right.\right.\right. \\
\left.\left.\left.\left.a_{\gamma_{1}}^{+} a_{\gamma_{1}^{\prime}}\right], a_{\gamma_{2}}^{+} a_{\gamma_{2}^{\prime}}\right], a_{\gamma_{3}}^{+} a_{\gamma_{3}^{\prime}}\right]\right\} .
\end{gathered}
$$

Applying (35) to $E_{11}\left(\bar{\omega}_{1}\right)$ again with $A \equiv \hbar \bar{\omega}_{1}$ and $B \equiv L_{\text {eq }}$ results in

$$
\begin{gathered}
E_{11}\left(\bar{\omega}_{1}\right) \\
=-\left(\frac{1}{\hbar \bar{\omega}_{1}}\right) T_{R}\left\{\rho _ { \mathrm { eq } } \left[G ( \overline { \omega } _ { 1 } ) \left[\left[L_{v} a_{\alpha}^{+} a_{\beta}, a_{\gamma_{1}}^{+} a_{\gamma_{1}^{\prime}}\right],\right.\right.\right. \\
\left.\left.\left.a_{\gamma_{2}}^{+} a_{\gamma_{2}^{\prime}}\right], L_{\mathrm{eq}} a_{\gamma_{3}}^{+} a_{\gamma_{3}^{\prime}}\right]\right\}
\end{gathered}
$$

in the second-order approximation in $L_{v}$, where we have used the (KCRI-I) ${ }_{1}$. Equation (87) can be calculated as follows:

$$
E_{11}\left(\bar{\omega}_{1}\right)=-\frac{V_{21}\left(\hbar \bar{\omega}_{1}\right)}{\left(\hbar \bar{\omega}_{1}+E_{\gamma_{3} \gamma_{3}^{\prime}}\right)},
$$

where we have used $L_{d} a_{\gamma_{3}}^{+} a_{\gamma_{3}^{\prime}}=E_{\gamma_{3} \gamma_{3}^{\prime}} a_{\gamma_{3}}^{+} a_{\gamma_{3}^{\prime}}$ and

$$
\begin{gathered}
V_{21}\left(\bar{\omega}_{1}\right)=T_{R}\left\{\rho _ { \mathrm { eq } } \left[G_{d}\left(\bar{\omega}_{1}\right)\left[\left[L_{v} a_{\alpha}^{+} a_{\beta}, a_{\gamma_{1}}^{+} a_{\gamma_{1}^{\prime}}\right], a_{\gamma_{2}}^{+} a_{\gamma_{2}^{\prime}}\right],\right.\right. \\
\left.\left.L_{v} a_{\gamma_{3}}^{+} a_{\gamma_{3}^{\prime}}\right]\right\} .
\end{gathered}
$$

KCRI-II is applied to $E_{12}\left(\bar{\omega}_{1}, \bar{\omega}_{2}\right)$ to calculate $E_{12}\left(\bar{\omega}_{1}, \bar{\omega}_{2}\right)$, resulting in

$$
\begin{aligned}
E_{12} & \left(\bar{\omega}_{1}, \bar{\omega}_{2}\right) \\
= & -\left(E_{\gamma_{2} \gamma_{2}^{\prime}}+E_{\gamma_{3} \gamma_{3}^{\prime}}\right) E_{1}\left(\bar{\omega}_{1}, \bar{\omega}_{2}\right) \\
& -\left(\hbar \bar{\omega}_{1}+E_{\gamma_{3} \gamma_{3}^{\prime}}\right)^{-1} V_{22}\left(\bar{\omega}_{1}, \bar{\omega}_{2}\right)-V_{23}\left(\bar{\omega}_{1}, \bar{\omega}_{2}\right)
\end{aligned}
$$

in the second-order approximation in $L_{v}$, where

$$
\begin{aligned}
& V_{22}\left(\bar{\omega}_{1}, \bar{\omega}_{2}\right) \\
& \equiv T_{R}\left\{\rho _ { \mathrm { eq } } \left[\left[G_{d}\left(\bar{\omega}_{12}\right)\left[L_{v} a_{\alpha}^{+} a_{\beta}, a_{\gamma_{1}}^{+} a_{\gamma_{1}^{\prime}}\right], L_{v} a_{\gamma_{2}}^{+} a_{\gamma_{2}^{\prime}}\right],\right.\right. \\
& \left.\left.\quad a_{\gamma_{3}}^{+} a_{\gamma_{3}^{\prime}}\right]\right\}, \\
& V_{23}\left(\bar{\omega}_{1}, \bar{\omega}_{2}\right) \\
& \equiv T_{R}\left\{\rho _ { \mathrm { eq } } \left[G _ { d } ( \overline { \omega } _ { 1 } ) \left[G_{d}\left(\bar{\omega}_{12}\right)\left[L_{v} a_{\alpha}^{+} a_{\beta}, a_{\gamma_{1}}^{+} a_{\gamma_{1}^{\prime}}\right],\right.\right.\right. \\
& \left.\left.\left.a_{\gamma_{2}}^{+} a_{\gamma_{2}^{\prime}}\right], L_{v} a_{\gamma_{3}}^{+} a_{\gamma_{3}^{\prime}}\right]\right\} .
\end{aligned}
$$

Therefore, inserting (87) and (90) into (84) gives

$$
\begin{aligned}
E_{1}\left(\bar{\omega}_{1}, \bar{\omega}_{2}\right)= & \left(\frac{-\left\{V_{21}\left(\bar{\omega}_{1}\right)+V_{22}\left(\bar{\omega}_{1}, \bar{\omega}_{2}\right)\right\}}{\hbar \bar{\omega}_{1}+E_{\gamma_{3} \gamma_{3}^{\prime}}}-V_{23}\left(\bar{\omega}_{1}, \bar{\omega}_{2}\right)\right) \\
& \times\left(\hbar \bar{\omega}_{12}+E_{\gamma_{2} \gamma_{2}^{\prime}}+E_{\gamma_{3} \gamma_{3}^{\prime}}\right)^{-1} .
\end{aligned}
$$

5.3.2. Calculation of $E_{2}\left(\bar{\omega}_{1}, \bar{\omega}_{2}, \bar{\omega}_{2}\right)$. Applying KCRI-III to (83) and using

$$
\begin{gathered}
T_{R}\left\{\rho _ { \mathrm { es } } \left[G ( \overline { \omega } _ { 1 } ) \left[G ( \overline { \omega } _ { 1 2 } ) \left[Q_{3}\left(\hbar \bar{\omega}_{123}-L_{\mathrm{eq}} Q_{3}\right)^{-1} L_{\nu} a_{\alpha}^{+} a_{\beta},\right.\right.\right.\right. \\
\left.\left.\left.\left.a_{\gamma_{1}}^{+} a_{\gamma_{1}^{\prime}}\right], a_{\gamma_{2}}^{+} a_{\gamma_{2}^{\prime}}\right], a_{\gamma_{3}}^{+} a_{\gamma_{3}^{\prime}}\right]\right\}=0
\end{gathered}
$$

result in

$$
\begin{aligned}
E_{2}\left(\bar{\omega}_{1}, \bar{\omega}_{2}, \bar{\omega}_{3}\right)= & -\frac{V_{24}\left(\bar{\omega}_{1}, \bar{\omega}_{2}, \bar{\omega}_{3}\right)+V_{25}\left(\bar{\omega}_{1}, \bar{\omega}_{2}, \bar{\omega}_{3}\right)}{\hbar \bar{\omega}_{1}+E_{\gamma_{3} \gamma_{3}^{\prime}}} \\
& -V_{26}\left(\bar{\omega}_{1}, \bar{\omega}_{2}, \bar{\omega}_{3}\right)
\end{aligned}
$$

in the second-order approximation in $L_{v}$, where

$$
\begin{aligned}
& V_{24}\left(\bar{\omega}_{1}, \bar{\omega}_{2}, \bar{\omega}_{3}\right) \\
& =T_{R}\left\{\rho _ { \mathrm { eq } } \left[\left[G _ { d } ( \overline { \omega } _ { 1 2 } ) \left[G_{d}\left(\bar{\omega}_{123}\right) L_{v} a_{\alpha}^{+} a_{\beta},\right.\right.\right.\right. \\
& \left.\left.\left.\left.L_{v} a_{\gamma_{1}}^{+} a_{\gamma_{1}^{\prime}}\right], a_{\gamma_{2}}^{+} a_{\gamma_{2}^{\prime}}\right], a_{\gamma_{3}}^{+} a_{\gamma_{3}^{\prime}}\right]\right\}, \\
& V_{25}\left(\bar{\omega}_{1}, \bar{\omega}_{2}, \bar{\omega}_{3}\right) \\
& =T_{R}\left\{\rho _ { \mathrm { eq } } \left[\left[G _ { d } ( \overline { \omega } _ { 1 2 } ) \left[G_{d}\left(\bar{\omega}_{123}\right) L_{v} a_{\alpha}^{+} a_{\beta},\right.\right.\right.\right. \\
& \left.\left.\left.\left.a_{\gamma_{1}}^{+} a_{\gamma_{1}^{\prime}}\right], L_{v} a_{\gamma_{2}}^{+} a_{\gamma_{2}^{\prime}}\right], a_{\gamma_{3}}^{+} a_{\gamma_{3}^{\prime}}\right]\right\}, \\
& \quad=T_{R}\left\{\rho _ { \mathrm { eq } } \left[G _ { d } ( \overline { \omega } _ { 1 } ) \left[G _ { d } ( \overline { \omega } _ { 1 2 } ) \left[G_{d}\left(\bar{\omega}_{123}\right) L_{v} a_{\alpha}^{+} a_{\beta},\right.\right.\right.\right. \\
& \left.\left.\left.\left.a_{\gamma_{1}}^{+} a_{\gamma_{1}^{\prime}}\right], a_{\gamma_{2}}^{+} a_{\gamma_{2}^{\prime}}\right], L_{v} a_{\gamma_{3}}^{+} a_{\gamma_{3}^{\prime}}\right]\right\} .
\end{aligned}
$$


Finally, inserting (93) and (95) into (81) gives

$$
\begin{aligned}
S_{3}\left(\bar{\omega}_{1}, \bar{\omega}_{2}, \bar{\omega}_{3}\right) & \\
=- & \bar{K}_{3}\left(\bar{\omega}_{1}, \bar{\omega}_{2}\right) \\
\times & {\left[\left(\frac{V_{21}\left(\bar{\omega}_{1}\right)+V_{22}\left(\bar{\omega}_{1}, \bar{\omega}_{2}\right)}{\hbar \bar{\omega}_{1}+E_{\gamma_{3} \gamma_{3}^{\prime}}}+V_{23}\left(\bar{\omega}_{1}, \bar{\omega}_{2}\right)\right)\right.} \\
& \times\left(\hbar \bar{\omega}_{12}+E_{\gamma_{2} \gamma_{2}^{\prime}}+E_{\gamma_{3} \gamma_{3}^{\prime}}\right)^{-1} \\
& +\frac{V_{24}\left(\bar{\omega}_{1}, \bar{\omega}_{2}, \bar{\omega}_{3}\right)+V_{25}\left(\bar{\omega}_{1}, \bar{\omega}_{2}, \bar{\omega}_{3}\right)}{\hbar \bar{\omega}_{1}+E_{\gamma_{3} \gamma_{3}^{\prime}}} \\
& \left.+V_{26}\left(\bar{\omega}_{1}, \bar{\omega}_{2}, \bar{\omega}_{3}\right)\right]
\end{aligned}
$$

in the second-order approximation in $L_{v}$, where

$$
\begin{gathered}
\bar{K}_{3}\left(\bar{\omega}_{1}, \bar{\omega}_{2}\right) \\
\equiv T_{R}\left\{\rho _ { \mathrm { eq } } \left[G _ { d } ( \overline { \omega } _ { 1 } ) \left[G_{d}\left(\bar{\omega}_{12}\right)\left[a_{\alpha}^{+} a_{\beta}, a_{\gamma_{1}}^{+} a_{\gamma_{1}^{\prime}}\right],\right.\right.\right. \\
\left.\left.\left.a_{\gamma_{2}}^{+} a_{\gamma_{2}^{\prime}}\right], a_{\gamma_{3}}^{+} a_{\gamma_{3}^{\prime}}\right]\right\} .
\end{gathered}
$$

\section{Lineshape Functions and Diagram Representations}

In this section, the lineshape functions for the electronphonon system are derived.

6.1. Linear Term. In (52) and (56),

$$
\left\langle a_{\alpha}^{+} a_{\beta}\right\rangle_{\gamma_{1}}=\left(f_{\alpha}-f_{\beta}\right) \delta_{\alpha \gamma_{1}^{\prime}} \delta_{\beta \gamma_{1}},
$$

where $f_{\alpha}$ is the Fermi distribution function for an electron in a state, $|\alpha\rangle$, with energy $E_{\alpha}$. Inserting (101) into (52) gives

$$
\begin{aligned}
R_{1}(\omega) & =T_{R}\left\{\rho_{\mathrm{eq}}\left[G(\bar{\omega}) a_{\alpha}^{+} a_{\beta}, a_{\gamma_{1}}^{+} a_{\gamma_{1}^{\prime}}\right]\right\} \\
& =\frac{\left(f_{\alpha}-f_{\beta}\right) \delta_{\alpha \gamma_{1}^{\prime}} \delta_{\beta \gamma_{1}}}{\hbar \bar{\omega}+E_{\beta \alpha}-\Gamma_{\beta \alpha}^{(0)}(\bar{\omega})} .
\end{aligned}
$$

Therefore, (46) becomes

$$
\sigma_{k l}(\omega)=e \sum_{\alpha, \beta} \frac{\left(j_{k}\right)_{\alpha \beta}\left(r_{l}\right)_{\beta \alpha}\left(f_{\beta}-f_{\alpha}\right)}{\hbar \bar{\omega}+E_{\beta \alpha}-\Gamma_{\beta \alpha}^{(0)}(\bar{\omega})}
$$

where the first lineshape function that contains the effect of the electron-phonon interaction is given as

$$
\Gamma_{\beta \alpha}^{(0)}(\bar{\omega})\left(f_{\beta}-f_{\alpha}\right) \equiv T_{R}\left\{\rho_{\mathrm{eq}}\left[G_{d}(\bar{\omega}) L_{v} a_{\alpha}^{+} a_{\beta}, L_{v} a_{\beta}^{+} a_{\alpha}\right]\right\}
$$

which can be calculated as follows:

$$
\begin{aligned}
& \Gamma_{\beta \alpha}^{(0)}(\bar{\omega})\left(f_{\beta}-f_{\alpha}\right) \\
& =\sum_{q} \sum_{\lambda, \lambda_{2}} T_{R}\left\{\rho _ { \mathrm { eq } } \left(G_{\lambda_{1} \beta}^{(+)}(\bar{\omega}) C_{\lambda_{1} \alpha}(q) C_{\lambda_{2} \beta}(-q)\right.\right. \\
& \times\left[b_{q} a_{\lambda_{1}}^{+} a_{\beta}, b_{q}^{+} a_{\lambda_{2}}^{+} a_{\alpha}\right] \\
& +G_{\lambda_{1} \beta}^{(-)}(\bar{\omega}) C_{\lambda_{1} \alpha}(q) C_{\lambda_{2} \beta}(-q) \\
& \times\left[b_{q}^{+} a_{\lambda_{1}}^{+} a_{\beta}, b_{q} a_{\lambda_{2}}^{+} a_{\alpha}\right] \\
& -G_{\alpha \lambda_{1}}^{(+)}(\bar{\omega}) C_{\beta \lambda_{1}}(q) C_{\lambda_{2} \beta}(-q) \\
& \times\left[b_{q} a_{\alpha}^{+} a_{\lambda_{1}}, b_{q}^{+} a_{\lambda_{2}}^{+} a_{\alpha}\right] \\
& -G_{\alpha \lambda_{1}}^{(-)}(\bar{\omega}) C_{\beta \lambda_{1}}(q) C_{\lambda_{2} \beta}(-q) \\
& \times\left[b_{q}^{+} a_{\alpha}^{+} a_{\lambda_{1}}, b_{q} a_{\lambda_{2}}^{+} a_{\alpha}\right] \\
& -G_{\lambda_{1} \beta}^{(+)}(\bar{\omega}) C_{\lambda_{1} \alpha}(q) C_{\alpha \lambda_{2}}(-q) \\
& \times\left[b_{q} a_{\lambda_{1}}^{+} a_{\beta}, b_{q}^{+} a_{\beta}^{+} a_{\lambda_{2}}\right] \\
& -G_{\lambda_{1} \beta}^{(-)}(\bar{\omega}) C_{\lambda_{1} \alpha}(q) C_{\alpha \lambda_{2}}(-q) \\
& \times\left[b_{q}^{+} a_{\lambda_{1}}^{+} a_{\beta}, b_{q} a_{\beta}^{+} a_{\lambda_{2}}\right] \\
& +G_{\alpha \lambda_{1}}^{(+)}(\bar{\omega}) C_{\beta \lambda_{1}}(q) C_{\alpha \lambda_{2}}(-q) \\
& \times\left[b_{q} a_{\alpha}^{+} a_{\lambda_{1}}, b_{q}^{+} a_{\beta}^{+} a_{\lambda_{2}}\right] \\
& +G_{\alpha \lambda_{1}}^{(-)}(\bar{\omega}) C_{\beta \lambda_{1}}(q) C_{\alpha \lambda_{2}}(-q) \\
& \left.\left.\times\left[b_{q}^{+} a_{\alpha}^{+} a_{\lambda_{1}}, b_{q} a_{\beta}^{+} a_{\lambda_{2}}\right]\right)\right\} \\
& =\sum_{q} \sum_{\lambda}\left[\left|C_{\beta \lambda}(q)\right|^{2}\right. \\
& \times\left\{G_{\lambda \alpha}^{(-)}(\bar{\omega}) P_{+}(\lambda, \alpha)+G_{\lambda \alpha}^{(+)}(\bar{\omega}) P_{-}(\lambda, \alpha)\right\} \\
& +\left|C_{\lambda \alpha}(q)\right|^{2} \\
& \left.\times\left\{G_{\beta \lambda}^{(-)}(\bar{\omega}) P_{+}(\beta, \lambda)+G_{\beta \lambda}^{(+)}(\bar{\omega}) P_{-}(\beta, \lambda)\right\}\right] .
\end{aligned}
$$

Here,

$$
\begin{gathered}
L_{v} a_{\alpha}^{+} a_{\beta}=\sum_{q} \sum_{\lambda}\left(b_{q}+b_{-q}^{+}\right)\left\{C_{\lambda \alpha}(q) a_{\lambda}^{+} a_{\beta}-C_{\beta \lambda}(q) a_{\alpha}^{+} a_{\lambda}\right\}, \\
L_{d} b_{q}^{ \pm} a_{\alpha}^{+} a_{\beta}=\left(E_{\alpha \beta} \pm \hbar \omega_{q}\right) b_{q}^{ \pm} a_{\alpha}^{+} a_{\beta}, \\
G_{d}(\bar{\omega}) b_{q}^{ \pm} a_{\alpha}^{+} a_{\beta}=\left(\hbar \bar{\omega}-E_{\alpha \beta} \mp \hbar \omega_{q}\right)^{-1} b_{q}^{ \pm} a_{\alpha}^{+} a_{\beta}, \\
{\left[b_{q} a_{\alpha}^{+} a_{\beta}, b_{q^{\prime}}^{+} a_{\gamma}^{+} a_{\delta}\right]=a_{\alpha}^{+} a_{\beta} a_{\gamma}^{+} a_{\delta} \delta_{q q^{\prime}}+b_{q^{\prime}}^{+} b_{q} a_{\alpha}^{+} a_{\delta} \delta_{\beta \gamma}} \\
-b_{q^{\prime}}^{+} b_{q} a_{\gamma}^{+} a_{\beta} \delta_{\alpha \delta},
\end{gathered}
$$




$$
\begin{gathered}
{\left[b_{q}^{+} a_{\alpha}^{+} a_{\beta}, b_{q^{\prime}} a_{\gamma}^{+} a_{\delta}\right]=-a_{\gamma}^{+} a_{\delta} a_{\alpha}^{+} a_{\beta} \delta_{q q^{\prime}}+b_{q}^{+} b_{q^{\prime}} a_{\alpha}^{+} a_{\delta} \delta_{\beta \gamma}} \\
-b_{q}^{+} b_{q^{\prime}} a_{\gamma}^{+} a_{\beta} \delta_{\alpha \delta}, \\
T_{R}\left\{\rho_{\mathrm{eq}} a_{\alpha}^{+} a_{\beta}\right\}=f_{\alpha} \delta_{\alpha \beta}, \\
T_{R}\left\{\rho_{\mathrm{eq}} b_{q}^{+} b_{q^{\prime}}\right\}=N_{q} \delta_{q q^{\prime}}, \\
T_{R}\left\{\rho_{\mathrm{eq}} a_{\alpha}^{+} a_{\beta} a_{\gamma}^{+} a_{\delta}\right\}=f_{\alpha}\left(1-f_{\gamma}\right) \delta_{\alpha \delta} \delta_{\beta \gamma}-f_{\alpha} f_{\gamma} \delta_{\alpha \beta} \delta_{\gamma \delta}
\end{gathered}
$$

were used, where $N_{q}$ is the Planck distribution function for phonons with energy $\hbar \omega_{q}$. In (105), the "energy denominator factor" is defined as

$$
G_{\beta \alpha}^{( \pm)}(\bar{\omega}) \equiv\left(\hbar \bar{\omega}+E_{\beta \alpha} \pm \hbar \omega_{q}\right)^{-1}
$$

and the "population factors" are defined as

$$
\begin{aligned}
& P_{+}(\alpha, \beta) \equiv\left(N_{q}+1\right) f_{\alpha}\left(1-f_{\beta}\right)-N_{q} f_{\beta}\left(1-f_{\alpha}\right), \\
& P_{-}(\alpha, \beta) \equiv N_{q} f_{\alpha}\left(1-f_{\beta}\right)-\left(N_{q}+1\right) f_{\beta}\left(1-f_{\alpha}\right) .
\end{aligned}
$$

The denominator in (103) represents the transition from the initial state $\beta$ to the final state $\alpha$ with a photon absorption of frequency $\omega$ and can be represented as Figure 1. If there is no phonon scattering, the lineshape of the conductivity would be like a delta function. On the other hand, the lineshape is broadened as electrons are scattered by phonons, so the lineshape function, $\Gamma_{\beta \alpha}^{(0)}(\bar{\omega})$, is included. Implicit states exist between the initial and final states because they are connected by $\Gamma_{\beta \alpha}^{(0)}(\bar{\omega})$, including the implicit states induced by electronphonon interactions; that is, $\Gamma_{\beta \alpha}^{(0)}(\bar{\omega})$ contains the implicit transitions between the initial (or final) and implicit states. An implicit state should be coupled with the initial (or final) state by a proper interaction factor in the case of an implicit transition between the final (or initial) and implicit states. $\Gamma_{\beta \alpha}^{(0)}(\bar{\omega})$ must also contain an energy denominator factor enforcing energy conservation in each implicit transition and a population factor justifying the implicit transition.

Although $\Gamma_{\beta \alpha}^{(0)}(\bar{\omega})$ is called the lineshape function, the lineshape must be calculated from (103), not directly from (105). $\Gamma_{\beta \alpha}^{(0)}(\bar{\omega})$ is represented by four diagrams in Figure 2. The two terms correspond to $\left|C_{\beta \lambda}(q)\right|^{2}$ and the other two terms correspond to $\left|C_{\lambda \alpha}(q)\right|^{2} .\left|C_{i j}(q)\right|^{2}$ is called the "interaction factor," through which momentum conservation is satisfied.

The physical meaning of the first term in (105) is as follows. $\left|C_{\beta \lambda}(q)\right|^{2}$ means that the implicit state $\lambda$ is coupled with the initial state $\beta$ by a phonon with a wave vector $q$. $\left|C_{\beta \lambda}(q)\right|^{2}$ is represented by a vertical dotted line (Figure $2(\mathrm{a})$ ). The states denoted by $\lambda$ are called implicit states because they are included only in lineshape function (see (105)), not in the conductivity (see (103)). $G_{\lambda \alpha}^{(-)}(\bar{\omega})$ means that the energy of the implicit state $\lambda$ is determined by the energy of the final state $\alpha$, photon energy $\hbar \omega$, and phonon energy $\hbar \omega_{q}$, so energy conservation can be satisfied; that is, $E_{\lambda}+\hbar \omega=$ $E_{\alpha}+\hbar \omega_{q} . G_{\lambda \alpha}^{(-)}(\bar{\omega})$ is represented by a horizontal solid line. The half circle with a rightward (leftward) arrow corresponds to photon absorption (emission) and the upper (lower) half circle corresponds to phonon emission (absorption). $P_{+}(\lambda, \alpha)$ means that the reverse implicit transition from the final state to the implicit state with photon emission and phonon absorption $\left(N_{q}\right)$ should be subtracted from the forward implicit transition from the implicit state to the final state with photon absorption and phonon emission $\left(1+N_{q}\right)$. This means that when an electron-phonon interaction is involved in an electron transition, the electron undergoes an implicit transition from an implicit state, which is coupled with the initial state, to the final state (vice versa) with photon absorption (emission) and phonon emission (absorption). The second term in (105) corresponds to phonon absorption (emission) for a forward (backward) process. The third and fourth terms in (105) correspond to the cases that the implicit state is coupled with the final state. Therefore, the transition from the initial state to the final state occurs via two implicit transitions and the implicit state is coupled with the initial or final states. We can represent the result in the diagram because the lineshape function contains an energy denominator, population, and interaction factors properly.

6.2. First-Order Nonlinear Term. In (62), $\left\langle a_{\alpha}^{+} a_{\beta}\right\rangle_{\gamma_{1} \gamma_{2}}$ can be calculated as

$$
\begin{aligned}
\left\langle a_{\alpha}^{+} a_{\beta}\right\rangle_{\gamma_{1} \gamma_{2}}= & T_{R}\left\{\rho_{\mathrm{eq}}\left[G\left(\bar{\omega}_{1}\right) a_{\alpha}^{+} a_{\gamma_{1}^{\prime}}, a_{\gamma_{2}}^{+} a_{\gamma_{2}^{\prime}}\right]\right\} \delta_{\beta \gamma_{1}} \\
& -T_{R}\left\{\rho_{\mathrm{eq}}\left[G\left(\bar{\omega}_{1}\right) a_{\gamma_{1}}^{+} a_{\beta}, a_{\gamma_{2}}^{+} a_{\gamma_{2}^{\prime}}\right]\right\} \delta_{\alpha \gamma_{1}^{\prime}} .
\end{aligned}
$$

Considering (102), (109) becomes

$$
\begin{aligned}
\left\langle a_{\alpha}^{+} a_{\beta}\right\rangle_{\gamma_{1} \gamma_{2}}= & \frac{\left(f_{\alpha}-f_{\gamma_{1}^{\prime}}\right) \delta_{\alpha \gamma_{2}^{\prime}} \delta_{\gamma_{1}^{\prime} \gamma_{2}}}{\hbar \bar{\omega}_{1}+E_{\gamma_{1}^{\prime} \alpha}-\Gamma_{\gamma_{1}^{\prime} \alpha}^{(0)}\left(\bar{\omega}_{1}\right)} \delta_{\beta \gamma_{1}} \\
& -\frac{\left(f_{\gamma_{1}}-f_{\beta}\right) \delta_{\gamma_{1} \gamma_{2}^{\prime}} \delta_{\beta \gamma_{2}}}{\hbar \bar{\omega}_{1}+E_{\beta \gamma_{1}}-\Gamma_{\beta \gamma_{1}}^{(0)}\left(\bar{\omega}_{1}\right)} \delta_{\alpha \gamma_{1}^{\prime}} .
\end{aligned}
$$

On the other hand, (75) can be calculated as follows:

$$
\begin{aligned}
\bar{K}_{2}\left(\bar{\omega}_{1}\right)= & \left(\hbar \bar{\omega}_{1}-E_{\alpha \gamma_{1}^{\prime}}\right)^{-1}\left(f_{\alpha}-f_{\gamma_{2}}\right) \delta_{\alpha \gamma_{2}^{\prime}} \delta_{\gamma_{1}^{\prime} \gamma_{2}} \delta_{\beta \gamma_{1}} \\
& -\left(\hbar \bar{\omega}_{1}-E_{\gamma_{1} \beta}\right)^{-1}\left(f_{\gamma_{1}}-f_{\gamma_{2}}\right) \delta_{\gamma_{1} \gamma_{2}^{\prime}} \delta_{\beta \gamma_{2}} \delta_{\alpha \gamma_{1}^{\prime}},
\end{aligned}
$$

where

$$
G_{d}(\bar{\omega}) a_{\alpha}^{+} a_{\beta}=\left(\hbar \bar{\omega}-E_{\alpha \beta}\right)^{-1} a_{\alpha}^{+} a_{\beta}
$$




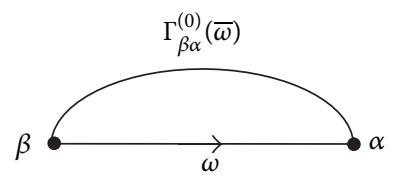

FIgURE 1: Schematic diagram of the energy denominator, $\left[\hbar \bar{\omega}+E_{\beta \alpha}-\Gamma_{\beta \alpha}^{(0)}(\bar{\omega})\right]^{-1}$, in $\sigma_{k l}(\omega)$.

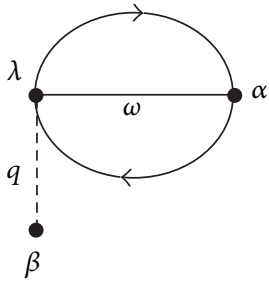

(a)

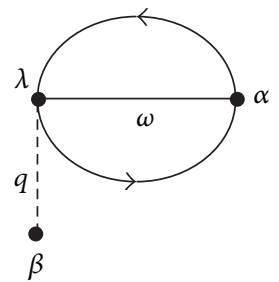

(b)

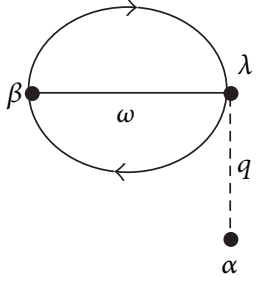

(c)

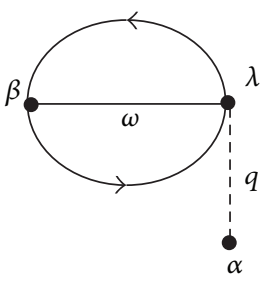

(d)

Figure 2: Schematic diagrams of (a) $\left|C_{\beta \lambda}(q)\right|^{2} G_{\lambda \alpha}^{(-)}(\bar{\omega}) P_{+}(\lambda, \alpha)$, (b) $\left|C_{\beta \lambda}(q)\right|^{2} G_{\lambda \alpha}^{(+)}(\bar{\omega}) P_{-}(\lambda, \alpha)$, (c) $\left|C_{\lambda \alpha}(q)\right|^{2} G_{\beta \lambda}^{(-)}(\bar{\omega}) P_{+}(\beta, \lambda)$, and $(\mathrm{d})$ $\left|C_{\lambda \alpha}(q)\right|^{2} G_{\beta \lambda}^{(+)}(\bar{\omega}) P_{-}(\beta, \lambda)$ in $\Gamma_{\beta \alpha}^{(0)}(\bar{\omega})$.

was used. Therefore, inserting (110) into (62) and using (74) and (111),

$$
\begin{aligned}
R_{2}\left(\bar{\omega}_{1}, \bar{\omega}_{2}\right) & T_{R}\left\{\rho_{\mathrm{eq}}\left[G\left(\bar{\omega}_{1}\right)\left[G\left(\bar{\omega}_{12}\right) a_{\alpha}^{+} a_{\beta}, a_{\gamma_{1}}^{+} a_{\gamma_{1}^{\prime}}\right], a_{\gamma_{2}}^{+} a_{\gamma_{2}^{\prime}}\right]\right\} \\
= & \frac{\left(f_{\beta}-f_{\gamma_{1}}\right) \delta_{\gamma_{1} \gamma_{2}^{\prime}} \delta_{\beta \gamma_{2}}}{\hbar \bar{\omega}_{1}+E_{\beta \gamma_{1}}-\Gamma_{\beta \gamma_{1}}^{(0)}\left(\bar{\omega}_{1}\right)} \cdot \frac{\delta_{\alpha \gamma_{1}^{\prime}}}{\hbar \bar{\omega}_{12}+E_{\beta \alpha}-\Gamma_{\beta \gamma_{1} \alpha}^{(11)}\left(\bar{\omega}_{1}, \bar{\omega}_{2}\right)} \\
& -\frac{\left(f_{\gamma_{1}^{\prime}}-f_{\alpha}\right) \delta_{\alpha \gamma_{2}^{\prime}} \delta_{\gamma_{1}^{\prime} \gamma_{2}}}{\hbar \bar{\omega}_{1}+E_{\gamma_{1}^{\prime} \alpha}-\Gamma_{\gamma_{1}^{\prime} \alpha}^{(0)}\left(\bar{\omega}_{1}\right)} \cdot \frac{\delta_{\beta \gamma_{1}}}{\hbar \bar{\omega}_{12}+E_{\beta \alpha}-\Gamma_{\beta \gamma_{1}^{\prime} \alpha}^{(12)}\left(\bar{\omega}_{1}, \bar{\omega}_{2}\right)},
\end{aligned}
$$

where

$$
\begin{aligned}
& \Gamma_{\beta \gamma_{1} \alpha}^{(11)}\left(\bar{\omega}_{1}, \bar{\omega}_{2}\right)\left(f_{\beta}-f_{\gamma_{1}}\right) \\
&=-\left(\hbar \bar{\omega}_{1}-E_{\gamma_{1} \beta}\right) \\
& \times\left[\frac{V_{11}\left(\bar{\omega}_{1}\right)+V_{12}\left(\bar{\omega}_{1}, \bar{\omega}_{2}\right)}{\hbar \bar{\omega}_{1}-E_{\gamma_{1} \beta}}+V_{13}\left(\bar{\omega}_{1}, \bar{\omega}_{2}\right)\right] \\
& \times \delta_{\gamma_{1} \gamma_{2}^{\prime}} \delta_{\beta \gamma_{2}} \delta_{\alpha \gamma_{1}^{\prime}} \\
& \approx-\left[V_{11}\left(\bar{\omega}_{1}\right)+V_{12}\left(\bar{\omega}_{1}, \bar{\omega}_{2}\right)\right] \delta_{\gamma_{1} \gamma_{2}^{\prime}} \delta_{\beta \gamma_{2}} \delta_{\alpha \gamma_{1}^{\prime}}, \\
& \Gamma_{\beta \gamma_{1}^{\prime} \alpha}^{(12)}\left(\bar{\omega}_{1}, \bar{\omega}_{2}\right)\left(f_{\gamma_{1}^{\prime}}-f_{\alpha}\right) \\
&=\left(\hbar \bar{\omega}_{1}-E_{\alpha \gamma_{1}^{\prime}}\right) \\
& \times\left[\frac{V_{11}\left(\bar{\omega}_{1}\right)+V_{12}\left(\bar{\omega}_{1}, \bar{\omega}_{2}\right)}{\hbar \bar{\omega}_{1}-E_{\alpha \gamma_{1}^{\prime}}}+V_{13}\left(\bar{\omega}_{1}, \bar{\omega}_{2}\right)\right] \\
& \times \delta_{\alpha \gamma_{2}^{\prime} \delta_{\gamma_{1}^{\prime} \gamma_{2}} \delta_{\beta \gamma_{1}}} \\
& \approx {\left[V_{11}\left(\bar{\omega}_{1}\right)+V_{12}\left(\bar{\omega}_{1}, \bar{\omega}_{2}\right)\right] \delta_{\alpha \gamma_{2}^{\prime}} \delta_{\gamma_{1}^{\prime} \gamma_{2}} \delta_{\beta \gamma_{1}} . }
\end{aligned}
$$

Here, we have neglected the $V_{13}$ values because we are interested in the results near the resonance points, $\hbar \bar{\omega}_{1}=E_{\gamma_{1} \beta}$ and $\hbar \bar{\omega}_{1}=E_{\alpha \gamma_{1}^{\prime}}$. Inserting (113) into (58) gives (see appendices (A.1) and (A.2) for details)

$$
\begin{aligned}
& \sigma_{k l m}\left(\omega_{1}, \omega_{2}\right) \\
& =e^{2} \sum_{\alpha, \beta, \gamma}\left[\frac{\left(f_{\beta}-f_{\gamma}\right)}{\hbar \bar{\omega}_{1}+E_{\beta \gamma}-\Gamma_{\beta \gamma}^{(0)}\left(\bar{\omega}_{1}\right)}\right. \\
& \times \frac{\left(j_{k}\right)_{\alpha \beta}\left(r_{l}\right)_{\gamma \alpha}\left(r_{m}\right)_{\beta \gamma}}{\hbar \bar{\omega}_{12}+E_{\beta \alpha}-\Gamma_{\beta \gamma \alpha}^{(11)}\left(\bar{\omega}_{1}, \bar{\omega}_{2}\right)} \\
& -\frac{\left(f_{\gamma}-f_{\alpha}\right)}{\hbar \bar{\omega}_{1}+E_{\gamma \alpha}-\Gamma_{\gamma \alpha}^{(0)}\left(\bar{\omega}_{1}\right)} \\
& \left.\times \frac{\left(j_{k}\right)_{\alpha \beta}\left(r_{l}\right)_{\beta \gamma}\left(r_{m}\right)_{\gamma \alpha}}{\hbar \bar{\omega}_{12}+E_{\beta \alpha}-\Gamma_{\beta \gamma \alpha}^{(12)}\left(\bar{\omega}_{1}, \bar{\omega}_{2}\right)}\right] \\
& =\sigma_{k l m}^{(I I-1)}\left(\omega_{1}, \omega_{2}\right)+\sigma_{k l m}^{(I I-2)}\left(\omega_{1}, \omega_{2}\right) .
\end{aligned}
$$

Considering (A.1) and (A.2) in the appendix, the lineshape functions are calculated as follows:

$$
\begin{aligned}
\Gamma_{\beta \gamma \alpha}^{(11)}\left(\bar{\omega}_{1}, \bar{\omega}_{2}\right)\left(f_{\beta}-f_{\gamma}\right) \\
=\sum_{q, \lambda}\left[\left|C_{\beta \lambda}(q)\right|^{2}\right. \\
\quad \times\left\{G_{\lambda \gamma}^{(-)}\left(\bar{\omega}_{1}\right) P_{+}(\lambda, \gamma)+G_{\lambda \gamma}^{(+)}\left(\bar{\omega}_{1}\right) P_{-}(\lambda, \gamma)\right\} \\
+\left|C_{\lambda \alpha}(q)\right|^{2} G_{\beta \lambda}^{(-)}\left(\bar{\omega}_{12}\right)\left\{P_{+}(\beta, \lambda)-P_{+}(\gamma, \lambda)\right\} \\
\left.+\left|C_{\lambda \alpha}(q)\right|^{2} G_{\beta \lambda}^{(+)}\left(\bar{\omega}_{12}\right)\left\{P_{-}(\beta, \lambda)-P_{-}(\gamma, \lambda)\right\}\right],
\end{aligned}
$$




$$
\begin{aligned}
\Gamma_{\beta \gamma \alpha}^{(12)}\left(\bar{\omega}_{1}, \bar{\omega}_{2}\right)\left(f_{\gamma}-f_{\alpha}\right) \\
=\sum_{q, \lambda}\left[\left|C_{\lambda \alpha}(q)\right|^{2}\right. \\
\times\left\{G_{\gamma \lambda}^{(-)}\left(\bar{\omega}_{1}\right) P_{+}(\gamma, \lambda)+G_{\gamma \lambda}^{(+)}\left(\bar{\omega}_{1}\right) P_{-}(\gamma, \lambda)\right\} \\
+\left|C_{\beta \lambda}(q)\right|^{2} G_{\lambda \alpha}^{(-)}\left(\bar{\omega}_{12}\right)\left\{P_{+}(\lambda, \alpha)-P_{+}(\lambda, \gamma)\right\} \\
\left.+\left|C_{\beta \lambda}(q)\right|^{2} G_{\lambda \alpha}^{(+)}\left(\bar{\omega}_{12}\right)\left\{P_{-}(\lambda, \alpha)-P_{-}(\lambda, \gamma)\right\}\right] .
\end{aligned}
$$

$\sigma_{k l m}^{(I I-1)}\left(\omega_{1}, \omega_{2}\right)$ and $\sigma_{k l m}^{(I I-2)}\left(\omega_{1}, \omega_{2}\right)$ in $(115)$ can be represented by Figure 3. An intermediate state, $\gamma$, exists because of the two photons processes. Therefore, the first-order nonlinear conductivity consists of two parts. The first one, called a $\left(\omega_{1} \mid \omega_{2}\right)$ process, is $\sigma_{k l m}^{(I I-1)}\left(\omega_{1}, \omega_{2}\right)$, which contains a photon absorption of frequency $\omega_{1}$ in an intermediate transition from the initial state $\beta$ to the intermediate state $\gamma$ (Figure 3(a)). The other one, called a $\left(\omega_{2} \mid \omega_{1}\right)$ process, is $\sigma_{k l m}^{(I I-2)}\left(\omega_{1}, \omega_{2}\right)$, which is an exchange process with $\omega_{1}$ and $\omega_{2}$ being exchanged; that is, it contains a photon absorption of frequency $\omega_{1}$ in an intermediate transition from the intermediate state to the final state, $\alpha$ (Figure 3(b)).

The first term, $\sigma_{k l m}^{I I-1}\left(\omega_{1}, \omega_{2}\right)$, contains two transition processes. The first is an intermediate transition from the initial state, $\beta$, to the intermediate state, $\gamma$, with a photon absorption of frequency $\omega_{1}$ and lineshape function, $\Gamma_{\beta \gamma}^{(0)}\left(\bar{\omega}_{1}\right)$, and the other is a direct transition from the initial state to the final state, $\alpha$, with two photon absorption of frequencies $\omega_{1}$ and $\omega_{2}$ and lineshape function $\Gamma_{\beta \gamma \alpha}^{(11)}\left(\bar{\omega}_{1}, \bar{\omega}_{2}\right)$. Note that $\hbar \bar{\omega}_{12}+E_{\beta \alpha}=$ $\hbar \bar{\omega}_{1}+E_{\beta \gamma}+\hbar \bar{\omega}_{2}+E_{\gamma \alpha}$. The dotted half circle in Figure 3(a) means that the $\gamma$ state is not connected to the $\alpha$ state by any lineshape function. Therefore, no implicit transition exists between the $\gamma$ and $\alpha$ states. The second term, $\sigma_{k l m}^{I I-2}\left(\omega_{1}, \omega_{2}\right)$, can be interpreted in a similar manner, where the $\gamma$ state is not connected to the $\beta$ state by any lineshape function. Figures 4 and 5 show schematic diagrams of the lineshape functions $\Gamma_{\beta \gamma \alpha}^{(11)}\left(\bar{\omega}_{1}, \bar{\omega}_{2}\right)$ and $\Gamma_{\beta \gamma \alpha}^{(12)}\left(\bar{\omega}_{1}, \bar{\omega}_{2}\right)$, respectively. The linear part $\Gamma^{(0)}(\bar{\omega})$ included in $\sigma_{k l m}\left(\omega_{1}, \omega_{2}\right)$ has the same form as (105).

Equation (116) can be interpreted as follows (Figure 4). Note that an intermediate state, $\gamma$, exists between the initial state, $\beta$, and final state, $\alpha$, in the first term of (115) (Figure 3(a)). With reasoning similar to that for the linear conductivity, there are four diagrams; two terms corresponding to $\left|C_{\beta \lambda}(q)\right|^{2}$ and the other two terms corresponding to $\left|C_{\lambda \alpha}(q)\right|^{2}$. The first term in (116) (Figure 4(a)) corresponds to a transition from the implicit state $\lambda$ to the intermediate state with a photon absorption of frequency $\omega_{1} \cdot G_{\lambda \gamma}^{(-)}\left(\bar{\omega}_{1}\right)$ (horizontal solid line) represents the intermediate transition and $\left|C_{\beta \lambda}(q)\right|^{2}$ (vertical dotted line) means that the implicit state is coupled with the initial state. The third term in (116) (Figure 4(c)) corresponds to a transition from the initial state to the implicit state with two photon absorption of frequencies $\omega_{1}$ and $\omega_{2} \cdot G_{\beta \lambda}^{(-)}\left(\bar{\omega}_{12}\right)$ represents the transition and
$\left|C_{\lambda \alpha}(q)\right|^{2}$ means that the implicit state is coupled with the final state. Note that $P_{+}(\gamma, \lambda)$ (dotted cicle) should be subtracted from $P_{+}(\beta, \lambda)$ because the intermediate state is not connected to the final state by any lineshape function (see Figure 3(a)). The second and fourth terms (Figures 4(b) and 4(d)) in (116) and the four terms in (117) (Figure 5) can be interpreted in a similar manner. Note that the implicit state in (117) is not connected to the initial state by any lineshape function (see Figure 3(b)).

6.3. Second-Order Nonlinear Term. In (80), $\left\langle a_{\alpha}^{+} a_{\beta}\right\rangle_{\gamma_{1} \gamma_{2} \gamma_{3}}$ can be calculated as follows:

$$
\begin{aligned}
& \left\langle a_{\alpha}^{+} a_{\beta}\right\rangle_{\gamma_{1} \gamma_{2} \gamma_{3}} \\
& =T_{R}\left\{\rho_{\mathrm{eq}}\left[G\left(\bar{\omega}_{1}\right)\left[G\left(\bar{\omega}_{12}\right) a_{\alpha}^{+} a_{\gamma_{1}^{\prime}}, a_{\gamma_{2}} a_{\gamma_{2}^{\prime}}\right], a_{\gamma_{3}} a_{\gamma_{3}^{\prime}}\right]\right\} \delta_{\beta \gamma_{1}} \\
& \quad-T_{R}\left\{\rho_{\mathrm{eq}}\left[G\left(\bar{\omega}_{1}\right)\left[G\left(\bar{\omega}_{12}\right) a_{\gamma_{1}}^{+} a_{\beta}, a_{\gamma_{2}} a_{\gamma_{2}^{\prime}}\right], a_{\gamma_{3}} a_{\gamma_{3}^{\prime}}\right]\right\} \delta_{\alpha \gamma_{1}^{\prime}}
\end{aligned}
$$

Considering (113), (118) becomes

$$
\begin{aligned}
& \left\langle a_{\alpha}^{+} a_{\beta}\right\rangle_{\gamma_{1} \gamma_{2} \gamma_{3}}=\frac{\left(f_{\gamma_{1}^{\prime}}-f_{\gamma_{2}}\right) \delta_{\gamma_{2} \gamma_{3}^{\prime} \delta_{\gamma_{1}^{\prime} \gamma_{3}}}}{\hbar \bar{\omega}_{1}+E_{\gamma_{1}^{\prime} \gamma_{2}}-\Gamma_{\gamma_{1}^{\prime} \gamma_{2}}^{(0)}\left(\bar{\omega}_{1}\right)} \\
& \times \frac{\delta_{\alpha \gamma_{2}^{\prime}} \delta_{\beta \gamma_{1}}}{\hbar \bar{\omega}_{12}+E_{\gamma_{1}^{\prime} \alpha}-\Gamma_{\gamma_{1}^{\prime} \gamma_{2} \alpha}^{(11)}\left(\bar{\omega}_{1}, \bar{\omega}_{2}\right)} \\
& -\frac{\left(f_{\gamma_{2}^{\prime}}-f_{\alpha}\right) \delta_{\alpha \gamma_{3}^{\prime}} \delta_{\gamma_{2}^{\prime} \gamma_{3}}}{\hbar \bar{\omega}_{1}+E_{\gamma_{2}^{\prime} \alpha}-\Gamma_{\gamma_{2}^{\prime} \alpha}^{(0)}\left(\bar{\omega}_{1}\right)} \\
& \times \frac{\delta_{\gamma_{1}^{\prime} \gamma_{2}} \delta_{\beta \gamma_{1}}}{\hbar \bar{\omega}_{12}+E_{\gamma_{1}^{\prime} \alpha}-\Gamma_{\gamma_{1}^{\prime} \gamma_{2}^{\prime} \alpha}^{(12)}\left(\bar{\omega}_{1}, \bar{\omega}_{2}\right)} \\
& -\frac{\left(f_{\beta}-f_{\gamma_{2}}\right) \delta_{\gamma_{2} \gamma_{3}^{\prime}} \delta_{\beta \gamma_{3}}}{\hbar \bar{\omega}_{1}+E_{\beta \gamma_{2}}-\Gamma_{\beta \gamma_{2}}^{(0)}\left(\bar{\omega}_{1}\right)} \\
& \times \frac{\delta_{\gamma_{1} \gamma_{2}^{\prime}} \delta_{\alpha \gamma_{1}^{\prime}}}{\hbar \bar{\omega}_{12}+E_{\beta \gamma_{1}}-\Gamma_{\beta \gamma_{2} \gamma_{1}}^{(11)}\left(\bar{\omega}_{1}, \bar{\omega}_{2}\right)} \\
& +\frac{\left(f_{\gamma_{2}^{\prime}}-f_{\gamma_{1}}\right) \delta_{\gamma_{1} \gamma_{3}^{\prime}} \delta_{\gamma_{2}^{\prime} \gamma_{3}}}{\hbar \bar{\omega}_{1}+E_{\gamma_{2}^{\prime} \gamma_{1}}-\Gamma_{\gamma_{2}^{\prime} \gamma_{1}}^{(0)}\left(\bar{\omega}_{1}\right)} \\
& \times \frac{\delta_{\beta \gamma_{2}} \delta_{\alpha \gamma_{1}^{\prime}}}{\hbar \bar{\omega}_{12}+E_{\beta \gamma_{1}}-\Gamma_{\beta \gamma_{2}^{\prime} \gamma_{1}}^{(12)}\left(\bar{\omega}_{1}, \bar{\omega}_{2}\right)} .
\end{aligned}
$$

On the other hand, (100) can be calculated as

$$
\begin{aligned}
\bar{K}_{3}\left(\bar{\omega}_{1}, \bar{\omega}_{2}\right)= & \left(\hbar \bar{\omega}_{12}-E_{\alpha \gamma_{1}^{\prime}}\right)^{-1} T_{R} \\
& \times\left\{\rho_{\mathrm{eq}}\left[G_{d}\left(\bar{\omega}_{1}\right) a_{\alpha}^{+} a_{\gamma_{2}^{\prime}}, a_{\gamma_{3}}^{+} a_{\gamma_{3}^{\prime}}\right]\right\} \delta_{\beta \gamma_{1}} \delta_{\gamma_{1}^{\prime} \gamma_{2}} \\
& -\left(\hbar \bar{\omega}_{12}-E_{\alpha \gamma_{1}^{\prime}}\right)^{-1} T_{R}
\end{aligned}
$$




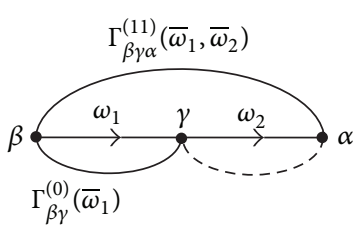

(a)

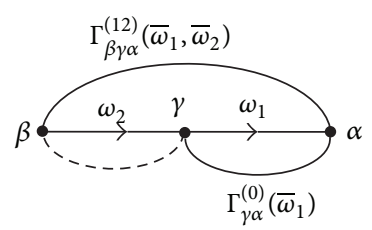

(b)

FIGURE 3: Schematic diagrams of the energy denominators in (a) $\sigma_{k l m}^{(I I-1)}\left(\bar{\omega}_{1}, \bar{\omega}_{2}\right)$ and (b) $\sigma_{k l m}^{(I I-2)}\left(\bar{\omega}_{1}, \bar{\omega}_{2}\right)$.

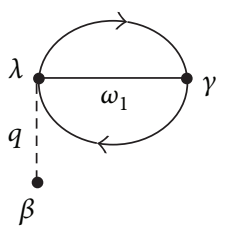

(a)

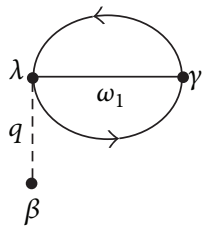

(b)

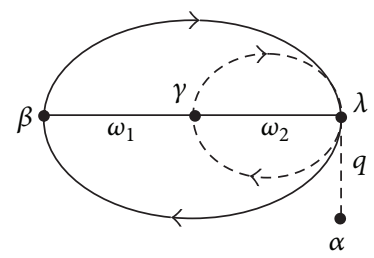

(c)

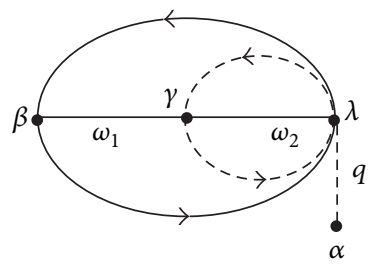

(d)

Figure 4: Schematic diagrams of (a) $\left|C_{\beta \lambda}(q)\right|^{2} G_{\lambda \gamma}^{(-)}\left(\bar{\omega}_{1}\right) P_{+}(\lambda, \gamma)$, (b) $\left|C_{\beta \lambda}(q)\right|^{2} G_{\lambda \gamma}^{(+)}\left(\bar{\omega}_{1}\right) P_{-}(\lambda, \gamma),(\mathrm{c})\left|C_{\lambda \alpha}(q)\right|^{2} G_{\beta \lambda}^{(-)}\left(\bar{\omega}_{12}\right)\left[P_{+}(\beta, \lambda)-P_{+}(\gamma, \lambda)\right]$, and (d) $\left|C_{\lambda \alpha}(q)\right|^{2} G_{\beta \lambda}^{(+)}\left(\bar{\omega}_{12}\right)\left[P_{-}(\beta, \lambda)-P_{-}(\gamma, \lambda)\right]$ in $\Gamma_{\beta \gamma \alpha}^{(11)}\left(\bar{\omega}_{1}, \bar{\omega}_{2}\right)$.

$$
\begin{aligned}
& \times\left\{\rho_{\mathrm{eq}}\left[G_{d}\left(\bar{\omega}_{1}\right) a_{\gamma_{2}}^{+} a_{\gamma_{1}^{\prime}}, a_{\gamma_{3}}^{+} a_{\gamma_{3}^{\prime}}\right]\right\} \delta_{\beta \gamma_{1}} \delta_{\alpha \gamma_{2}^{\prime}} \\
& -\left(\hbar \bar{\omega}_{12}-E_{\gamma_{1} \beta}\right)^{-1} T_{R} \\
& \times\left\{\rho_{\mathrm{eq}}\left[G_{d}\left(\bar{\omega}_{1}\right) a_{\gamma_{1}}^{+} a_{\gamma_{2}^{\prime}}, a_{\gamma_{3}}^{+} a_{\gamma_{3}^{\prime}}\right]\right\} \delta_{\alpha \gamma_{1}^{\prime}} \delta_{\beta \gamma_{2}} \\
& +\left(\hbar \bar{\omega}_{12}-E_{\gamma_{1} \beta}\right)^{-1} T_{R} \\
& \times\left\{\rho_{\mathrm{eq}}\left[G_{d}\left(\bar{\omega}_{1}\right) a_{\gamma_{2}}^{+} a_{\beta}, a_{\gamma_{3}}^{+} a_{\gamma_{3}^{\prime}}\right]\right\} \delta_{\alpha \gamma_{1}^{\prime}} \delta_{\gamma_{1} \gamma_{2}^{\prime}},
\end{aligned}
$$

where we have used (112). Applying (112) to (120) gives

$$
\begin{aligned}
\bar{K}_{3}\left(\bar{\omega}_{1}, \bar{\omega}_{2}\right)= & \left(\hbar \bar{\omega}_{12}-E_{\alpha \gamma_{1}^{\prime}}\right)^{-1}\left(\hbar \bar{\omega}_{1}-E_{\alpha \gamma_{2}^{\prime}}\right)^{-1} \\
& \times\left(f_{\alpha}-f_{\gamma_{2}^{\prime}}\right) \delta_{\alpha \gamma_{3}^{\prime}} \delta_{\gamma_{2}^{\prime} \gamma_{3}} \delta_{\beta \gamma_{1}} \delta_{\gamma_{1}^{\prime} \gamma_{2}} \\
& -\left(\hbar \bar{\omega}_{12}-E_{\alpha \gamma_{1}^{\prime}}\right)^{-1}\left(\hbar \bar{\omega}_{1}-E_{\gamma_{2} \gamma_{1}^{\prime}}\right)^{-1} \\
& \times\left(f_{\gamma_{2}}-f_{\gamma_{1}^{\prime}}\right) \delta_{\gamma_{2} \gamma_{3}^{\prime}} \delta_{\gamma_{1}^{\prime} \gamma_{3}} \delta_{\beta \gamma_{1}} \delta_{\alpha \gamma_{2}^{\prime}} \\
& -\left(\hbar \bar{\omega}_{12}-E_{\gamma_{1} \beta}\right)^{-1}\left(\hbar \bar{\omega}_{1}-E_{\gamma_{1} \gamma_{2}^{\prime}}\right)^{-1} \\
& \times\left(f_{\gamma_{1}}-f_{\gamma_{2}^{\prime}}\right) \delta_{\gamma_{1} \gamma_{3}^{\prime}} \delta_{\gamma_{2}^{\prime} \gamma_{3}} \delta_{\alpha \gamma_{1}^{\prime}} \delta_{\beta \gamma_{2}} \\
& +\left(\hbar \bar{\omega}_{12}-E_{\gamma_{1} \beta}\right)^{-1}\left(\hbar \bar{\omega}_{1}-E_{\gamma_{2} \beta}\right)^{-1} \\
& \times\left(f_{\gamma_{2}}-f_{\beta}\right) \delta_{\gamma_{2} \gamma_{3}^{\prime}} \delta_{\beta \gamma_{3}} \delta_{\alpha \gamma_{1}^{\prime}} \delta_{\gamma_{1} \gamma_{2}^{\prime}} .
\end{aligned}
$$

Therefore, inserting (119) into (80) and using (99) and (121) yield

$$
\begin{aligned}
R_{3}\left(\omega_{1}, \omega_{2}, \omega_{3}\right)= & -\frac{\left(f_{\beta}-f_{\gamma_{2}}\right) \delta_{\gamma_{2} \gamma_{3}^{\prime}} \delta_{\beta \gamma_{3}}}{\hbar \bar{\omega}_{1}+E_{\beta \gamma_{2}}-\Gamma_{\beta \gamma_{2}}^{(0)}\left(\bar{\omega}_{1}\right)} \\
& \times \frac{\delta_{\gamma_{1} \gamma_{2}^{\prime}} \delta_{\alpha \gamma_{1}^{\prime}}}{\hbar \bar{\omega}_{12}+E_{\beta \gamma_{1}}-\Gamma_{\beta \gamma_{2} \gamma_{1}}^{(11)}\left(\bar{\omega}_{1}, \bar{\omega}_{2}\right)}
\end{aligned}
$$

$$
\begin{aligned}
& \times \frac{1}{\hbar \bar{\omega}_{123}+E_{\beta \alpha}-\Gamma_{\beta \gamma_{2} \gamma_{1} \alpha}^{(21)}\left(\bar{\omega}_{1}, \bar{\omega}_{2}, \bar{\omega}_{3}\right)} \\
& +\frac{\left(f_{\gamma_{2}^{\prime}}-f_{\gamma_{1}}\right) \delta_{\gamma_{1} \gamma_{3}^{\prime}} \delta_{\gamma_{2}^{\prime} \gamma_{3}}}{\hbar \bar{\omega}_{1}+E_{\gamma_{2}^{\prime} \gamma_{1}}-\Gamma_{\gamma_{2}^{\prime} \gamma_{1}}^{(0)}\left(\bar{\omega}_{1}\right)} \\
& \times \frac{\delta_{\beta \gamma_{2}} \delta_{\alpha \gamma_{1}^{\prime}}}{\hbar \bar{\omega}_{12}+E_{\beta \gamma_{1}}-\Gamma_{\beta \gamma_{2}^{\prime} \gamma_{1}}^{(12)}\left(\bar{\omega}_{1}, \bar{\omega}_{2}\right)}
\end{aligned}
$$$$
\times \frac{1}{\hbar \bar{\omega}_{123}+E_{\beta \alpha}-\Gamma_{\beta \gamma_{2}^{\prime} \gamma_{1} \alpha}^{(22)}\left(\bar{\omega}_{1}, \bar{\omega}_{2}, \bar{\omega}_{3}\right)}
$$$$
+\frac{\left(f_{\gamma_{1}^{\prime}}-f_{\gamma_{2}}\right) \delta_{\gamma_{2} \gamma_{3}^{\prime}} \delta_{\gamma_{1}^{\prime} \gamma_{3}}}{\hbar \bar{\omega}_{1}+E_{\gamma_{1}^{\prime} \gamma_{2}}-\Gamma_{\gamma_{1}^{\prime} \gamma_{2}}^{(0)}\left(\bar{\omega}_{1}\right)}
$$$$
\times \frac{\delta_{\alpha \gamma_{2}^{\prime}} \delta_{\beta \gamma_{1}}}{\hbar \bar{\omega}_{12}+E_{\gamma_{1}^{\prime} \alpha}-\Gamma_{\gamma_{1}^{\prime} \gamma_{2} \alpha}^{(11)}\left(\bar{\omega}_{1}, \bar{\omega}_{2}\right)}
$$$$
\times \frac{1}{\hbar \bar{\omega}_{123}+E_{\beta \alpha}-\Gamma_{\beta \gamma_{1}^{\prime} \gamma_{2} \alpha}^{(23)}\left(\bar{\omega}_{1}, \bar{\omega}_{2}, \bar{\omega}_{3}\right)}
$$$$
-\frac{\left(f_{\gamma_{2}^{\prime}}-f_{\alpha}\right) \delta_{\alpha \gamma_{3}^{\prime}} \delta_{\gamma_{2}^{\prime} \gamma_{3}}}{\hbar \bar{\omega}_{1}+E_{\gamma_{2}^{\prime} \alpha}-\Gamma_{\gamma_{2}^{\prime} \alpha}^{(0)}\left(\bar{\omega}_{1}\right)}
$$$$
\times \frac{\delta_{\gamma_{1}^{\prime} \gamma_{2}} \delta_{\beta \gamma_{1}}}{\hbar \bar{\omega}_{12}+E_{\gamma_{1}^{\prime} \alpha}-\Gamma_{\gamma_{1}^{\prime} \gamma_{2}^{\prime} \alpha}^{(12)}\left(\bar{\omega}_{1}, \bar{\omega}_{2}\right)}
$$$$
\times \frac{1}{\hbar \bar{\omega}_{123}+E_{\beta \alpha}-\Gamma_{\beta \gamma_{1}^{\prime} \gamma_{2}^{\prime} \alpha}^{(24)}\left(\bar{\omega}_{1}, \bar{\omega}_{2}, \bar{\omega}_{3}\right)},
$$ 


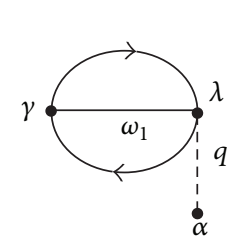

(a)

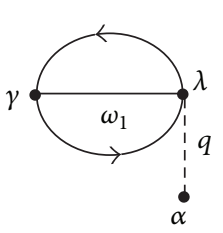

(b)

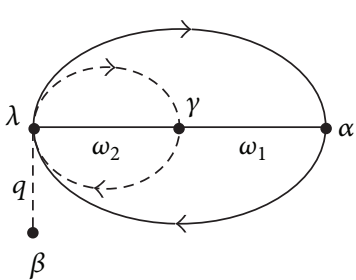

(c)

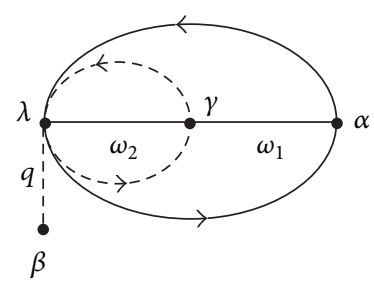

(d)

Figure 5: Schematic diagrams of (a) $\left|C_{\lambda \alpha}(q)\right|^{2} G_{\gamma \lambda}^{(-)}\left(\bar{\omega}_{1}\right) P_{+}(\gamma, \lambda),(\mathrm{b})\left|C_{\lambda \alpha}(q)\right|^{2} G_{\gamma \lambda}^{(+)}\left(\bar{\omega}_{1}\right) P_{-}(\gamma, \lambda),(\mathrm{c})\left|C_{\beta \lambda}(q)\right|^{2} G_{\lambda \alpha}^{(-)}\left(\bar{\omega}_{12}\right)\left[P_{+}(\lambda, \alpha)-P_{+}(\lambda, \gamma)\right]$, and (d) $\left|C_{\beta \lambda}(q)\right|^{2} G_{\lambda \alpha}^{(+)}\left(\bar{\omega}_{12}\right)\left[P_{-}(\lambda, \alpha)-P_{-}(\lambda, \gamma)\right]$ in $\Gamma_{\beta \gamma \alpha}^{(12)}\left(\bar{\omega}_{1}, \bar{\omega}_{2}\right)$.

where the four lineshape functions are given as follows:

$$
\begin{aligned}
& \Gamma_{\beta \gamma_{2} \gamma_{1} \alpha}^{(21)}\left(\bar{\omega}_{1}, \bar{\omega}_{2}, \bar{\omega}_{3}\right)\left(f_{\beta}-f_{\gamma_{2}}\right) \\
& =\left(\hbar \bar{\omega}_{12}-E_{\gamma_{1} \beta}\right)\left(\hbar \bar{\omega}_{1}-E_{\gamma_{2} \beta}\right) \\
& \times\left[\left(\frac{V_{21}\left(\bar{\omega}_{1}\right)+V_{22}\left(\bar{\omega}_{1}, \bar{\omega}_{2}\right)}{\hbar \bar{\omega}_{1}-E_{\gamma_{2} \beta}}+V_{23}\left(\bar{\omega}_{1}, \bar{\omega}_{2}\right)\right)\right. \\
& \times\left(\hbar \bar{\omega}_{12}+E_{\gamma_{3} \gamma_{2}^{\prime}}\right)^{-1} \\
& +\frac{V_{24}\left(\bar{\omega}_{1}, \bar{\omega}_{2}, \bar{\omega}_{3}\right)}{\hbar \bar{\omega}_{1}-E_{\gamma_{2} \beta}}+V_{25}\left(\bar{\omega}_{1}, \bar{\omega}_{2}, \bar{\omega}_{3}\right) \\
& \left.+V_{26}\left(\bar{\omega}_{1}, \bar{\omega}_{2}, \bar{\omega}_{3}\right)\right] \delta_{\gamma_{2} \gamma_{3}^{\prime}} \delta_{\beta \gamma_{3}} \delta_{\alpha \gamma_{1}^{\prime}} \delta_{\gamma_{1} \gamma_{2}^{\prime}} \\
& \approx\left[V_{21}\left(\bar{\omega}_{1}\right)+V_{22}\left(\bar{\omega}_{1}, \bar{\omega}_{2}\right)\right. \\
& \left.+\left(\hbar \bar{\omega}_{12}-E_{\gamma_{1} \beta}\right) V_{24}\left(\bar{\omega}_{1}, \bar{\omega}_{2}, \bar{\omega}_{3}\right)\right] \delta_{\gamma_{2} \gamma_{3}^{\prime}} \delta_{\beta \gamma_{3}} \delta_{\alpha \gamma_{1}^{\prime}} \delta_{\gamma_{1} \gamma_{2}^{\prime}}, \\
& \Gamma_{\beta \gamma_{2}^{\prime} \gamma_{1} \alpha}^{(22)}\left(\bar{\omega}_{1}, \bar{\omega}_{2}, \bar{\omega}_{3}\right)\left(f_{\gamma_{2}^{\prime}}-f_{\gamma_{1}}\right) \\
& =-\left(\hbar \bar{\omega}_{12}-E_{\gamma_{1} \beta}\right)\left(\hbar \bar{\omega}_{1}-E_{\gamma_{1} \gamma_{2}^{\prime}}\right) \\
& \times\left[\left(\frac{V_{21}\left(\bar{\omega}_{1}\right)+V_{22}\left(\bar{\omega}_{1}, \bar{\omega}_{2}\right)}{\hbar \bar{\omega}_{1}-E_{\gamma_{1} \gamma_{2}^{\prime}}}+V_{23}\left(\bar{\omega}_{1}, \bar{\omega}_{2}\right)\right)\right. \\
& \times\left(\hbar \bar{\omega}_{12}+E_{\gamma_{2} \gamma_{3}^{\prime}}\right)^{-1} \\
& +\frac{V_{24}\left(\bar{\omega}_{1}, \bar{\omega}_{2}, \bar{\omega}_{3}\right)}{\hbar \bar{\omega}_{1}-E_{\gamma_{1} \gamma_{2}^{\prime}}}+V_{25}\left(\bar{\omega}_{1}, \bar{\omega}_{2}, \bar{\omega}_{3}\right) \\
& \left.+V_{26}\left(\bar{\omega}_{1}, \bar{\omega}_{2}, \bar{\omega}_{3}\right)\right] \delta_{\gamma_{1} \gamma_{3}^{\prime}} \delta_{\gamma_{2}^{\prime} \gamma_{3}} \delta_{\alpha \gamma_{1}^{\prime}} \delta_{\beta \gamma_{2}} \\
& \approx-\left[V_{21}\left(\bar{\omega}_{1}\right)+V_{22}\left(\bar{\omega}_{1}, \bar{\omega}_{2}\right)\right. \\
& \left.+\left(\hbar \bar{\omega}_{12}-E_{\gamma_{1} \beta}\right) V_{24}\left(\bar{\omega}_{1}, \bar{\omega}_{2}, \bar{\omega}_{3}\right)\right] \delta_{\gamma_{1} \gamma_{3}^{\prime}} \delta_{\gamma_{2}^{\prime} \gamma_{3}} \delta_{\alpha \gamma_{1}^{\prime}} \delta_{\beta \gamma_{2}},
\end{aligned}
$$

$$
\begin{aligned}
& \Gamma_{\beta \gamma_{1}^{\prime} \gamma_{2} \alpha}^{(23)}\left(\bar{\omega}_{1}, \bar{\omega}_{2}, \bar{\omega}_{3}\right)\left(f_{\gamma_{1}^{\prime}}-f_{\gamma_{2}}\right) \\
& =-\left(\hbar \bar{\omega}_{12}-E_{\alpha \gamma_{1}^{\prime}}\right)\left(\hbar \bar{\omega}_{1}-E_{\gamma_{2} \gamma_{1}^{\prime}}\right) \\
& \times\left[\left(\frac{V_{21}\left(\bar{\omega}_{1}\right)+V_{22}\left(\bar{\omega}_{1}, \bar{\omega}_{2}\right)}{\hbar \bar{\omega}_{1}-E_{\gamma_{2} \gamma_{1}^{\prime}}}+V_{23}\left(\bar{\omega}_{1}, \bar{\omega}_{2}\right)\right)\right. \\
& \times\left(\hbar \bar{\omega}_{12}+E_{\gamma_{3} \gamma_{2}^{\prime}}\right)^{-1} \\
& +\frac{V_{24}\left(\bar{\omega}_{1}, \bar{\omega}_{2}, \bar{\omega}_{3}\right)}{\hbar \bar{\omega}_{1}-E_{\gamma_{2} \gamma_{1}^{\prime}}}+V_{25}\left(\bar{\omega}_{1}, \bar{\omega}_{2}, \bar{\omega}_{3}\right) \\
& \left.+V_{26}\left(\bar{\omega}_{1}, \bar{\omega}_{2}, \bar{\omega}_{3}\right)\right] \delta_{\gamma_{2} \gamma_{3}^{\prime}} \delta_{\gamma_{1}^{\prime} \gamma_{3}} \delta_{\beta \gamma_{1}} \delta_{\alpha \gamma_{2}^{\prime}} \\
& \approx-\left[V_{21}\left(\bar{\omega}_{1}\right)+V_{22}\left(\bar{\omega}_{1}, \bar{\omega}_{2}\right)\right. \\
& \left.+\left(\hbar \bar{\omega}_{12}-E_{\alpha \gamma_{1}^{\prime}}\right) V_{24}\left(\bar{\omega}_{1}, \bar{\omega}_{2}, \bar{\omega}_{3}\right)\right] \delta_{\gamma_{2} \gamma_{3}^{\prime}} \delta_{\gamma_{1}^{\prime} \gamma_{3}} \delta_{\beta \gamma_{1}} \delta_{\alpha \gamma_{2}^{\prime}}, \\
& \Gamma_{\beta \gamma_{1}^{\prime} \gamma_{2}^{\prime} \alpha}^{(24)}\left(\bar{\omega}_{1}, \bar{\omega}_{2}, \bar{\omega}_{3}\right)\left(f_{\gamma_{2}^{\prime}}-f_{\alpha}\right) \\
& =\left(\hbar \bar{\omega}_{12}-E_{\alpha \gamma_{1}^{\prime}}\right)\left(\hbar \bar{\omega}_{1}-E_{\alpha \gamma_{2}^{\prime}}\right) \\
& \times\left[\left(\frac{V_{21}\left(\bar{\omega}_{1}\right)+V_{22}\left(\bar{\omega}_{1}, \bar{\omega}_{2}\right)}{\hbar \bar{\omega}_{1}-E_{\alpha \gamma_{2}^{\prime}}}+V_{23}\left(\bar{\omega}_{1}, \bar{\omega}_{2}\right)\right)\right. \\
& \times\left(\hbar \bar{\omega}_{12}+E_{\gamma_{2} \gamma_{3}^{\prime}}\right)^{-1} \\
& +\frac{V_{24}\left(\bar{\omega}_{1}, \bar{\omega}_{2}, \bar{\omega}_{3}\right)}{\hbar \bar{\omega}_{1}-E_{\alpha \gamma_{2}^{\prime}}}+V_{25}\left(\bar{\omega}_{1}, \bar{\omega}_{2}, \bar{\omega}_{3}\right) \\
& \left.+V_{26}\left(\bar{\omega}_{1}, \bar{\omega}_{2}, \bar{\omega}_{3}\right)\right] \delta_{\alpha \gamma_{3}^{\prime}} \delta_{\gamma_{2}^{\prime} \gamma_{3}} \delta_{\beta \gamma_{1}} \delta_{\gamma_{1}^{\prime} \gamma_{2}} \\
& \approx\left[V_{21}\left(\bar{\omega}_{1}\right)+V_{22}\left(\bar{\omega}_{1}, \bar{\omega}_{2}\right)\right. \\
& \left.+\left(\hbar \bar{\omega}_{12}-E_{\alpha \gamma_{1}^{\prime}}\right) V_{24}\left(\bar{\omega}_{1}, \bar{\omega}_{2}, \bar{\omega}_{3}\right)\right] \delta_{\alpha \gamma_{3}^{\prime}} \delta_{\gamma_{2}^{\prime} \gamma_{3}} \delta_{\beta \gamma_{1}} \delta_{\gamma_{1}^{\prime} \gamma_{2}} .
\end{aligned}
$$

Here, $V_{23}, V_{25}$, and $V_{26}$ values were neglected because the resonance phenomena are considered. 
Finally, inserting (122) into (76) gives (see appendices (A.3), (A.4), and (A.5) for details)

$$
\begin{aligned}
& \sigma_{k l m n}\left(\omega_{1}, \omega_{2}, \omega_{3}\right) \\
& =e^{3} \sum_{\alpha, \beta} \sum_{\gamma_{1}, \gamma_{2}}\left[\frac{f_{\beta}-f_{\gamma_{1}}}{\hbar \bar{\omega}_{1}+E_{\beta \gamma_{1}}-\Gamma_{\beta \gamma_{1}}^{(0)}\left(\bar{\omega}_{1}\right)}\right. \\
& \times \frac{\left(j_{k}\right)_{\alpha \beta}\left(r_{n}\right)_{\beta \gamma_{1}}}{\hbar \bar{\omega}_{12}+E_{\beta \gamma_{2}}-\Gamma_{\beta \gamma_{1} \gamma_{2}}^{(11)}\left(\bar{\omega}_{1}, \bar{\omega}_{2}\right)} \\
& \times \frac{\left(r_{m}\right)_{\gamma_{1} \gamma_{2}}\left(r_{l}\right)_{\gamma_{2} \alpha}}{\hbar \bar{\omega}_{123}+E_{\beta \alpha}-\Gamma_{\beta \gamma_{1} \gamma_{2} \alpha}^{(21)}\left(\bar{\omega}_{1}, \bar{\omega}_{2}, \bar{\omega}_{3}\right)} \\
& -\frac{f_{\gamma_{1}}-f_{\gamma_{2}}}{\hbar \bar{\omega}_{1}+E_{\gamma_{1} \gamma_{2}}-\Gamma_{\gamma_{1} \gamma_{2}}^{(0)}\left(\bar{\omega}_{1}\right)} \\
& \times \frac{\left(j_{k}\right)_{\alpha \beta}\left(r_{m}\right)_{\beta \gamma_{1}}}{\hbar \bar{\omega}_{12}+E_{\beta \gamma_{2}}-\Gamma_{\beta \gamma_{1} \gamma_{2}}^{(12)}\left(\bar{\omega}_{1}, \bar{\omega}_{2}\right)} \\
& \times \frac{\left(r_{n}\right)_{\gamma_{2} \gamma_{1}}\left(r_{l}\right)_{\gamma_{1} \alpha}}{\hbar \bar{\omega}_{123}+E_{\beta \alpha}-\Gamma_{\beta \gamma_{1} \gamma_{2} \alpha}^{(22)}\left(\bar{\omega}_{1}, \bar{\omega}_{2}, \bar{\omega}_{3}\right)} \\
& -\frac{f_{\gamma_{1}}-f_{\gamma_{2}}}{\hbar \bar{\omega}_{1}+E_{\gamma_{1} \gamma_{2}}-\Gamma_{\gamma_{1} \gamma_{2}}^{(0)}\left(\bar{\omega}_{1}\right)} \\
& \times \frac{\left(j_{k}\right)_{\alpha \beta}\left(r_{l}\right)_{\beta \gamma_{1}}}{\hbar \bar{\omega}_{12}+E_{\gamma_{1} \alpha}-\Gamma_{\gamma_{1} \gamma_{2} \alpha}^{(11)}\left(\bar{\omega}_{1}, \bar{\omega}_{2}\right)} \\
& \times \frac{\left(r_{n}\right)_{\gamma_{1} \gamma_{2}}\left(r_{m}\right)_{\gamma_{2} \alpha}}{\hbar \bar{\omega}_{123}+E_{\beta \alpha}-\Gamma_{\beta \gamma_{1} \gamma_{2} \alpha}^{(23)}\left(\bar{\omega}_{1}, \bar{\omega}_{2}, \bar{\omega}_{3}\right)} \\
& +\frac{f_{\gamma_{2}}-f_{\alpha}}{\hbar \bar{\omega}_{1}+E_{\gamma_{2} \alpha}-\Gamma_{\gamma_{2} \alpha}^{(0)}\left(\bar{\omega}_{1}\right)} \\
& \times \frac{\left(j_{k}\right)_{\alpha \beta}\left(r_{l}\right)_{\beta \gamma_{1}}}{\hbar \bar{\omega}_{12}+E_{\gamma_{1} \alpha}-\Gamma_{\gamma_{1} \gamma_{2} \alpha}^{(12)}\left(\bar{\omega}_{1}, \bar{\omega}_{2}\right)} \\
& \left.\times \frac{\left(r_{m}\right)_{\gamma_{1} \gamma_{2}}\left(r_{n}\right)_{\gamma_{2} \alpha}}{\hbar \bar{\omega}_{123}+E_{\beta \alpha}-\Gamma_{\beta \gamma_{1} \gamma_{2} \alpha}^{(24)}\left(\bar{\omega}_{1}, \bar{\omega}_{2}, \bar{\omega}_{3}\right)}\right] \\
& =\sigma_{k l m n}^{(I I I-1)}\left(\omega_{1}, \omega_{2}, \omega_{3}\right)+\sigma_{k l m n}^{(I I I-2)}\left(\omega_{1}, \omega_{2}, \omega_{3}\right) \\
& +\sigma_{k l m n}^{(I I I-3)}\left(\omega_{1}, \omega_{2}, \omega_{3}\right)+\sigma_{k l m n}^{(I I I-4)}\left(\omega_{1}, \omega_{2}, \omega_{3}\right)
\end{aligned}
$$

where, considering (A.3)-(A.5) in the appendix, the lineshape functions are calculated as follows:

$$
\begin{aligned}
\Gamma_{\beta \gamma_{1} \alpha_{2} \beta}^{(21)}\left(\bar{\omega}_{1}, \bar{\omega}_{2}, \bar{\omega}_{3}\right)\left(f_{\beta}-f_{\gamma_{1}}\right) \\
=\sum_{q, \lambda}\left[\left|C_{\beta \lambda}(q)\right|^{2}\right. \\
\quad \times\left\{G_{\lambda \gamma_{1}}^{(-)}\left(\bar{\omega}_{1}\right) P_{+}\left(\lambda, \gamma_{1}\right)+G_{\lambda \gamma_{1}}^{(+)}\left(\bar{\omega}_{1}\right) P_{-}\left(\lambda, \gamma_{1}\right)\right\}
\end{aligned}
$$

$$
\begin{aligned}
& +\left|C_{\lambda \alpha}(q)\right|^{2} G_{\beta \lambda}^{(-)}\left(\bar{\omega}_{123}\right)\left\{P_{+}(\beta, \lambda)-P_{+}\left(\gamma_{1}, \lambda\right)\right\} \\
& \left.+\left|C_{\lambda \alpha}(q)\right|^{2} G_{\beta \lambda}^{(+)}\left(\bar{\omega}_{123}\right)\left\{P_{-}(\beta, \lambda)-P_{-}\left(\gamma_{1}, \lambda\right)\right\}\right],
\end{aligned}
$$$$
\Gamma_{\beta \gamma_{1} \gamma_{2} \alpha}^{(22)}\left(\bar{\omega}_{1}, \bar{\omega}_{2}, \bar{\omega}_{3}\right)\left(f_{\gamma_{1}}-f_{\gamma_{2}}\right)
$$$$
=\sum_{q, \lambda}\left[\left|C_{\beta \lambda}(q)\right|^{2} G_{\lambda \gamma_{2}}^{(-)}\left(\bar{\omega}_{12}\right)\left\{P_{+}\left(\lambda, \gamma_{2}\right)-P_{+}\left(\lambda, \gamma_{1}\right)\right\}\right.
$$$$
+\left|C_{\beta \lambda}(q)\right|^{2} G_{\lambda \gamma_{2}}^{(+)}\left(\bar{\omega}_{12}\right)\left\{P_{-}\left(\lambda, \gamma_{2}\right)-P_{-}\left(\lambda, \gamma_{1}\right)\right\}
$$$$
+\left|C_{\lambda \alpha}(q)\right|^{2} G_{\beta \lambda}^{(-)}\left(\bar{\omega}_{123}\right)\left\{P_{+}\left(\gamma_{1}, \lambda\right)-P_{+}\left(\gamma_{2}, \lambda\right)\right\}
$$$$
\left.+\left|C_{\lambda \alpha}(q)\right|^{2} G_{\beta \lambda}^{(+)}\left(\bar{\omega}_{123}\right)\left\{P_{-}\left(\gamma_{1}, \lambda\right)-P_{-}\left(\gamma_{2}, \lambda\right)\right\}\right],
$$

$$
\begin{aligned}
\Gamma_{\beta \gamma_{1} \gamma_{2} \alpha}^{(23)}\left(\bar{\omega}_{1}, \bar{\omega}_{2}, \bar{\omega}_{3}\right)\left(f_{\gamma_{1}}-f_{\gamma_{2}}\right) & \\
=\sum_{q, \lambda} & {\left[\left|C_{\lambda \alpha}(q)\right|^{2} G_{\gamma_{1} \lambda}^{(-)}\left(\bar{\omega}_{12}\right)\left\{P_{+}\left(\gamma_{1}, \lambda\right)-P_{+}\left(\gamma_{2}, \lambda\right)\right\}\right.} \\
& +\left|C_{\lambda \alpha}(q)\right|^{2} G_{\gamma_{1} \lambda}^{(+)}\left(\bar{\omega}_{12}\right)\left\{P_{-}\left(\gamma_{1}, \lambda\right)-P_{-}\left(\gamma_{2}, \lambda\right)\right\} \\
& +\left|C_{\beta \lambda}(q)\right|^{2} G_{\lambda \alpha}^{(-)}\left(\bar{\omega}_{123}\right)\left\{P_{+}\left(\lambda, \gamma_{2}\right)-P_{+}\left(\lambda, \gamma_{1}\right)\right\} \\
& \left.+\left|C_{\beta \lambda}(q)\right|^{2} G_{\lambda \alpha}^{(+)}\left(\bar{\omega}_{123}\right)\left\{P_{-}\left(\lambda, \gamma_{2}\right)-P_{-}\left(\lambda, \gamma_{1}\right)\right\}\right],
\end{aligned}
$$

$$
\begin{aligned}
\Gamma_{\beta \gamma_{1} \gamma_{2} \alpha}^{(24)}\left(\bar{\omega}_{1}, \bar{\omega}_{2}, \bar{\omega}_{3}\right)\left(f_{\gamma_{2}}-f_{\alpha}\right) \\
=\sum_{q, \lambda}\left[\left|C_{\lambda \alpha}(q)\right|^{2}\right. \\
\quad \times\left\{G_{\gamma_{2} \lambda}^{(-)}\left(\bar{\omega}_{1}\right) P_{+}\left(\gamma_{2}, \lambda\right)+G_{\gamma_{2} \lambda}^{(+)}\left(\bar{\omega}_{1}\right) P_{-}\left(\gamma_{2}, \lambda\right)\right\} \\
+\left|C_{\beta \lambda}(q)\right|^{2} G_{\lambda \alpha}^{(-)}\left(\bar{\omega}_{123}\right)\left\{P_{+}(\lambda, \alpha)-P_{+}\left(\lambda, \gamma_{2}\right)\right\} \\
\left.+\left|C_{\beta \lambda}(q)\right|^{2} G_{\lambda \alpha}^{(+)}\left(\bar{\omega}_{123}\right)\left\{P_{-}(\lambda, \alpha)-P_{-}\left(\lambda, \gamma_{2}\right)\right\}\right] .
\end{aligned}
$$

The physical meaning of (124) is clear. Two intermediate states exist because there are three photon processes, and thus, four terms in the second-order nonlinear conductivity exist (Figure 6). With reasoning similar to that for the firstorder nonlinear conductivity, there are $\left(\omega_{1} \mid \omega_{2}\right)$ and $\left(\omega_{2} \mid \omega_{1}\right)$ processes, which consist of two processes each. The first term, $\sigma_{k l m n}^{(I I I-1)}\left(\omega_{1}, \omega_{2}, \omega_{3}\right)$, contains a photon absorption process of frequency $\omega_{3}$ and the $\left(\omega_{1} \mid \omega_{2}\right)$ process (Figure 6(a)). The third term, $\sigma_{k l m n}^{(I I I-3)}\left(\omega_{1}, \omega_{2}, \omega_{3}\right)$, contains the $\left(\omega_{1} \mid \omega_{2}\right)$ process exchanged with a photon absorption process of frequency $\omega_{3}$ (Figure 6(c)). The second and fourth terms, $\sigma_{k l m n}^{(I I I-2)}\left(\omega_{1}, \omega_{2}, \omega_{3}\right)$ and $\sigma_{k l m n}^{(I I I-4)}\left(\omega_{1}, \omega_{2}, \omega_{3}\right)$, contain the $\left(\omega_{2}\right.$ $\left.\omega_{1}\right)$ process (Figures 6(b) and 6(d)). 


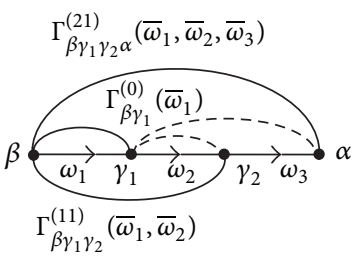

(a)

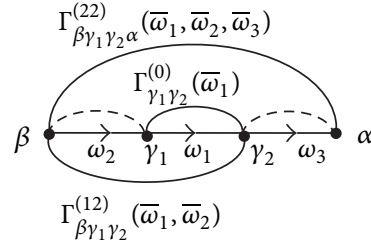

(b)

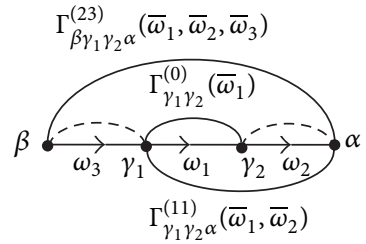

(c)

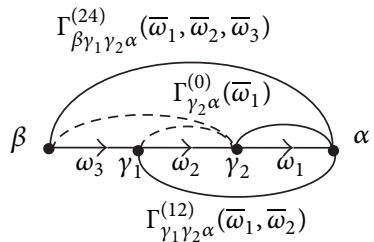

(d)

FIGURE 6: Schematic diagrams of the energy denominators in (a) $\sigma_{k l m n}^{(I I I-1)}\left(\bar{\omega}_{1}, \bar{\omega}_{2}, \bar{\omega}_{3}\right),(\mathrm{b}) \sigma_{k l m n}^{(I I I-2)}\left(\bar{\omega}_{1}, \bar{\omega}_{2}, \bar{\omega}_{3}\right),(\mathrm{c}) \sigma_{k l m n}^{(I I I-3)}\left(\bar{\omega}_{1}, \bar{\omega}_{2}, \bar{\omega}_{3}\right)$, and $(\mathrm{d})$ $\sigma_{k l m n}^{(I I I-4)}\left(\bar{\omega}_{1}, \bar{\omega}_{2}, \bar{\omega}_{3}\right)$.

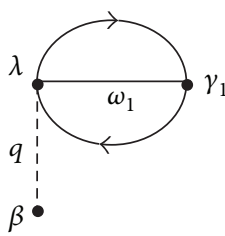

(a)

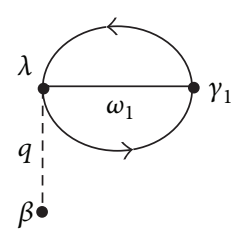

(b)

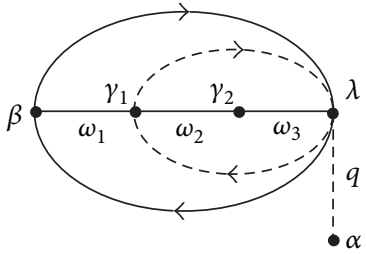

(c)

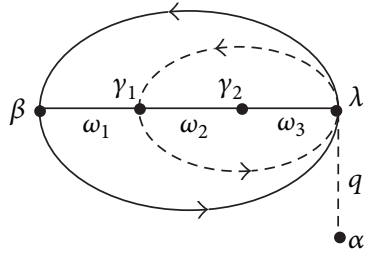

(d)

Figure 7: Schematic diagrams of (a) $\left|C_{\beta \lambda}(q)\right|^{2} G_{\lambda \gamma_{1}}^{(-)}\left(\bar{\omega}_{1}\right) P_{+}\left(\lambda, \gamma_{1}\right),(\mathrm{b})\left|C_{\beta \lambda}(q)\right|^{2} G_{\lambda \gamma_{1}}^{(+)}\left(\bar{\omega}_{1}\right) P_{-}\left(\lambda, \gamma_{1}\right),(\mathrm{c})\left|C_{\lambda \alpha}(q)\right|^{2} G_{\beta \lambda}^{(-)}\left(\bar{\omega}_{123}\right)\left[P_{+}(\beta, \lambda)-P_{+}\left(\gamma_{1}, \lambda\right)\right]$, and (d) $\left|C_{\lambda \alpha}(q)\right|^{2} G_{\beta \lambda}^{(+)}\left(\bar{\omega}_{123}\right)\left[P_{-}(\beta, \lambda)-P_{-}\left(\gamma_{1}, \lambda\right)\right]$ in $\Gamma_{\beta \gamma_{1} \gamma_{2} \alpha}^{(21)}\left(\bar{\omega}_{1}, \bar{\omega}_{2}, \bar{\omega}_{3}\right)$.

The first term, $\sigma_{k l m n}^{(I I I-1)}\left(\omega_{1}, \omega_{2}, \omega_{3}\right)$, contains three transition processes (Figure 6(a)). The first transition is an intermediate transition from the initial state $\beta$ to an intermediate state $\gamma_{1}$, with a photon absorption of frequency $\omega_{1}$ and lineshape function $\Gamma_{\beta \gamma_{1}}^{(0)}\left(\bar{\omega}_{1}\right)$. The second transition is an intermediate transition from the initial state to an intermediate state $\gamma_{2}$, with two photons absorption of frequencies $\omega_{1}$ and $\omega_{2}$ and lineshape function $\Gamma_{\beta \gamma_{1} \gamma_{2}}^{(11)}\left(\bar{\omega}_{1}, \bar{\omega}_{2}\right)$. The third transition is a direct transition from the initial state to the final state $\alpha$, with three photon absorption of frequencies $\omega_{1}, \omega_{2}$, and $\omega_{3}$ and lineshape function $\Gamma_{\beta \gamma_{1} \gamma_{2} \alpha}^{(21)}\left(\bar{\omega}_{1}, \bar{\omega}_{2}, \bar{\omega}_{3}\right)$. Note that $\hbar \bar{\omega}_{123}+E_{\beta \alpha}=$ $\hbar \bar{\omega}_{1}+E_{\beta \gamma_{1}}+\hbar \bar{\omega}_{2}+E_{\gamma_{1} \gamma_{2}}+\hbar \bar{\omega}_{3}+E_{\gamma_{2} \alpha}$. The dotted half circle in Figure 6(a) means that the $\gamma_{1}$ state is not connected to the $\gamma_{2}$ and $\alpha$ states by any lineshape function. On the other hand, the $\gamma_{1}$ and $\gamma_{2}$ states in the second term, $\sigma_{k l m n}^{(I I I-2)}\left(\omega_{1}, \omega_{2}, \omega_{3}\right)$, are not connected to the $\beta$ and $\alpha$ states, respectively, by any lineshape function. The other terms in (124) can be interpreted in a similar manner. Figures 7, 8, 9, and 10 show schematic diagrams of the four lineshape functions (see $(125)-(128))$ in (124). The linear part, $\Gamma^{(0)}\left(\bar{\omega}_{1}\right)$, included in $\sigma_{k l m n}\left(\omega_{1}, \omega_{2}, \omega_{3}\right)$ has the same form as (105) and the firstorder nonlinear parts, $\Gamma^{(1 i)}\left(\bar{\omega}_{1}, \bar{\omega}_{2}\right)$, has the same forms as (116) or (117).

Equation (125) (Figure 7) can be interpreted by (116) and (126) can be interpreted as follows (Figure 8). Like the linear and first-order nonlinear conductivities, there are four diagrams. The first two terms correspond to $\left|C_{\beta \lambda}(q)\right|^{2}$ and the other two terms correspond to $\left|C_{\lambda \alpha}(q)\right|^{2}$. The first term (Figure 8(a)) represents a transition from the implicit state $\lambda$ to an intermediate state $\gamma_{2}$, with two photon absorption of frequencies $\omega_{1}$ and $\omega_{2} . G_{\lambda \gamma_{2}}^{(-)}\left(\bar{\omega}_{12}\right)$ (horizontal solid line) represents the transition and $\left|C_{\beta \lambda}(q)\right|^{2}$ (vertical dotted line) means that the implicit state is coupled with the initial state, $\beta$. Note that $P_{+}\left(\lambda, \gamma_{1}\right)$ (dotted circle) should be subtracted from $P_{+}\left(\lambda, \gamma_{2}\right)$ because the intermediate state, $\gamma_{1}$, is not connected to the initial state by any lineshape function (see Figure 6(b)) in $\left(\omega_{2} \mid \omega_{1}\right)$ process. The third term (Figure 8(c)) corresponds to a transition from the initial state to the final state, $\alpha$, with three photon absorption of frequencies $\omega_{1}, \omega_{2}$, and $\omega_{3}$. Note that the intermediate state, $\gamma_{2}$, is not connected to the final state by any lineshape function (see Figure 6(b)). Therefore, $P_{+}\left(\gamma_{2}, \lambda\right)$ should be subtracted from $P_{+}\left(\gamma_{1}, \lambda\right)$, not from $P_{+}(\beta, \lambda)$, because the $\gamma_{1}$ state is also not connected to the initial state, $\beta$, by any lineshape function (see Figure 6(b)). Equations (127) and (128) can be interpreted in a similar manner (Figures 9 and 10).

\section{Concluding Remarks}

So far, we presented a method for deriving the nonlinear optical conductivity tensors for a system of electrons interacting with phonons using the PR method. The standard inductive inference will be required for a more rigorous proof of the general form of KCRI. The use of this method in the present problem, however, was beyond the scope of this study because the general-order term contains a large number of elementary factors. Consequently, this study used a simplified version of the induction instead, which states that the proposition KCRI of the $n$th kind is valid for all positive integers, $n$, if it holds for $n=1,2$, and 3 . This method is justifiable because the structure of KCRI and the population factor are iterative.

Nonlinear optics is concerned with the phenomena resulting from light-induced changes in the optical properties 


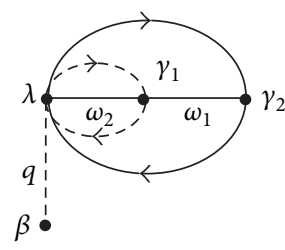

(a)

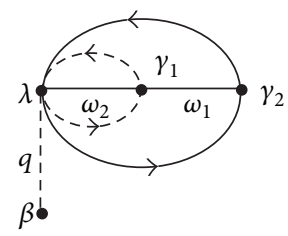

(b)

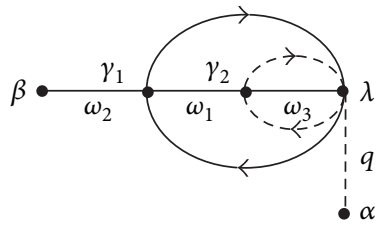

(c)

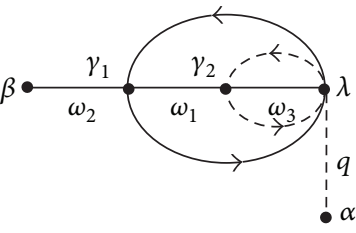

(d)

Figure 8: Schematic diagrams of (a) $\left|C_{\beta \lambda}(q)\right|^{2} G_{\lambda \gamma_{1}}^{(-)}\left(\bar{\omega}_{12}\right)\left[P_{+}\left(\lambda, \gamma_{2}\right)-P_{+}\left(\lambda, \gamma_{1}\right)\right]$, (b) $\left|C_{\beta \lambda}(q)\right|^{2} G_{\lambda \gamma_{2}}^{(+)}\left(\bar{\omega}_{12}\right)\left[P_{-}\left(\lambda, \gamma_{2}\right)-P_{-}\left(\lambda, \gamma_{1}\right)\right], \quad(\mathrm{c})$ $\left|C_{\lambda \alpha}(q)\right|^{2} G_{\beta \lambda}^{(-)}\left(\bar{\omega}_{123}\right)\left[P_{+}\left(\gamma_{1}, \lambda\right)-P_{+}\left(\gamma_{2}, \lambda\right)\right]$, and $(\mathrm{d})\left|C_{\lambda \alpha}(q)\right|^{2} G_{\beta \lambda}^{(+)}\left(\bar{\omega}_{123}\right)\left[P_{-}\left(\gamma_{1}, \lambda\right)-P_{-}\left(\gamma_{2}, \lambda\right)\right]$ in $\Gamma_{\beta \gamma_{1} \gamma_{2} \alpha}^{(22)}\left(\bar{\omega}_{1}, \bar{\omega}_{2}, \bar{\omega}_{3}\right)$.

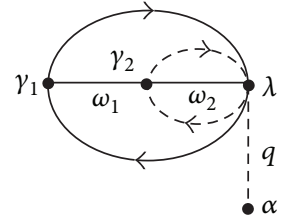

(a)

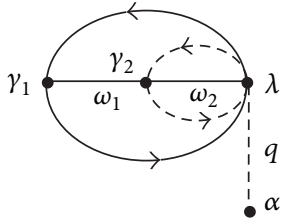

(b)

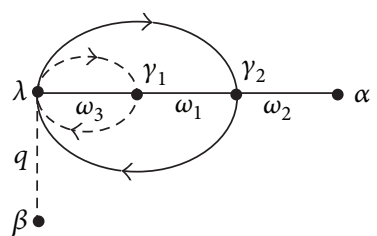

(c)

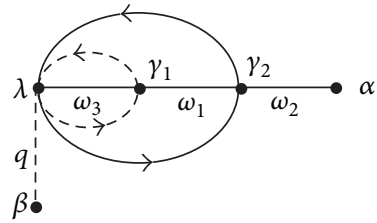

(d)

Figure 9: Schematic diagrams of (a) $\left|C_{\lambda \alpha}(q)\right|^{2} G_{\gamma_{1} \lambda}^{(-)}\left(\bar{\omega}_{12}\right)\left[P_{+}\left(\gamma_{1}, \lambda\right)-P_{+}\left(\gamma_{2}, \lambda\right)\right]$, (b) $\left|C_{\lambda \alpha}(q)\right|^{2} G_{\gamma_{1} \lambda}^{(+)}\left(\bar{\omega}_{12}\right)\left[P_{-}\left(\gamma_{1}, \lambda\right)-P_{-}\left(\gamma_{2}, \lambda\right)\right], \quad(\mathrm{c})$ $\left|C_{\beta \lambda}(q)\right|^{2} G_{\lambda \alpha}^{(-)}\left(\bar{\omega}_{123}\right)\left[P_{+}\left(\lambda, \gamma_{2}\right)-P_{+}\left(\lambda, \gamma_{1}\right)\right]$, and (d) $\left|C_{\beta \lambda}(q)\right|^{2} G_{\lambda \alpha}^{(+)}\left(\bar{\omega}_{123}\right)\left[P_{-}\left(\lambda, \gamma_{2}\right)-P_{-}\left(\lambda, \gamma_{1}\right)\right]$ in $\Gamma_{\beta \gamma_{1} \gamma_{2} \alpha}^{(23)}\left(\bar{\omega}_{1}, \bar{\omega}_{2}, \bar{\omega}_{3}\right)$.

of a system, the first-order nonlinear part being the simplest example. This study considers the second harmonic generation (SHG) as a proper example, in which the second parts of $\Gamma_{\beta \gamma \alpha}^{(11)}\left(\omega_{1}, \omega_{2}\right)$ and $\Gamma_{\beta \gamma \alpha}^{(12)}\left(\omega_{1}, \omega_{2}\right)$ play an important role with $\omega_{12}=2 \omega_{1}$. Note that a small fraction is converted to light at $\omega_{12}=2 \omega_{1}$ if an incident beam at frequency $\omega_{1}$ is focused on a proper sample. The two fractions can be filtered technically using a prism. Although there have been many studies on SHG in systems of electrons interacting with phonons [3436 , less attention has been paid to how the absorption and emission of photons and phonons occur in each process. The present work aims to clear up the physical cause and effect of the processes. These results will be improved if radiation-induced inharmonic phonon modes are included in the consideration. This part with a derivation of the highorder nonlinear term will be examined in future.

The projection operators used in this study are generally applicable because they satisfy the projection criterion (see statements below (51)). The lineshape functions in the linear, first-, and second-order nonlinear optical conductivities include the electron distribution functions to satisfy the population criterion as well as energy denominator factors enforcing energy conservation and the interaction factors coupling the implicit state with the initial, final, or intermediate states. Therefore, the phonon emissions and absorptions as well as photon absorptions and emissions in all electron transition processes can be presented in an organized manner and the results can be represented in diagrams, through which a physical intuition to quantum dynamics of electron transition in a solid can be obtained. These diagrams are quite different from the temperature diagrams with imaginary times in the Feynman-like scheme [37-42]. In the present scheme, the contribution of each $V_{i j}$ term to the lineshape function (or the damping term) is the sum of all weighted topologically distinct diagrams. Therefore, the present scheme can be categorized as another Feynmanlike scheme. In conclusion, we claim that a theory, which is generally applicable and satisfies the population criterion, can be obtained using the PR method. Formulations of other electron transition phenomena might be obtained using this method.

\section{Appendix}

\section{Calculation of $V_{i j}$ Values}

Using (106) properly, (68), (72), (89), (91), and (96) can be calculated. Here, we neglect the terms including $C_{\alpha \alpha}( \pm q)$ and $G_{\alpha \alpha}^{( \pm)}(\bar{\omega})$ because we consider only the electron-phonon interaction given by (2) and (3):

$$
\begin{aligned}
& V_{11}\left(\bar{\omega}_{1}\right) \\
& =\sum_{q} \sum_{\lambda_{1}, \lambda_{2}} T_{R} \\
& \times\left\{\rho _ { \mathrm { eq } } \left(G_{\lambda_{1} \gamma_{1}^{\prime}}^{(+)}\left(\bar{\omega}_{1}\right) C_{\lambda_{1} \alpha}(q) C_{\lambda_{2} \gamma_{2}}(-q) \delta_{\beta \gamma_{1}}\right.\right. \\
& \times\left[b_{q} a_{\lambda_{1}}^{+} a_{\gamma_{1}^{\prime}}, b_{q}^{+} a_{\lambda_{2}}^{+} a_{\gamma_{2}^{\prime}}\right] \\
& +G_{\lambda_{1} \gamma_{1}^{\prime}}^{(-)}\left(\bar{\omega}_{1}\right) C_{\lambda_{1} \alpha}(q) C_{\lambda_{2} \gamma_{2}}(-q) \delta_{\beta \gamma_{1}} \\
& \times\left[b_{q}^{+} a_{\lambda_{1}}^{+} a_{\gamma_{1}^{\prime}}, b_{q} a_{\lambda_{2}}^{+} a_{\gamma_{2}^{\prime}}\right] \\
& -G_{\lambda_{1} \gamma_{1}^{\prime}}^{(+)}\left(\bar{\omega}_{1}\right) C_{\lambda_{1} \alpha}(q) C_{\gamma_{2}^{\prime} \lambda_{2}}(-q) \delta_{\beta \gamma_{1}} \\
& \times\left[b_{q} a_{\lambda_{1}}^{+} a_{\gamma_{1}^{\prime}}, b_{q}^{+} a_{\gamma_{2}}^{+} a_{\lambda_{2}}\right] \\
& -G_{\lambda_{1} \gamma_{1}^{\prime}}^{(-)}\left(\bar{\omega}_{1}\right) C_{\lambda_{1} \alpha}(q) C_{\gamma_{2}^{\prime} \lambda_{2}}(-q) \delta_{\beta \gamma_{1}} \\
& \times\left[b_{q}^{+} a_{\lambda_{1}}^{+} a_{\gamma_{1}^{\prime}}, b_{q} a_{\gamma_{2}}^{+} a_{\lambda_{2}}\right]
\end{aligned}
$$




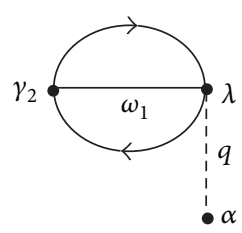

(a)

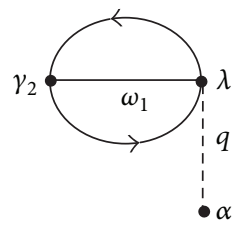

(b)

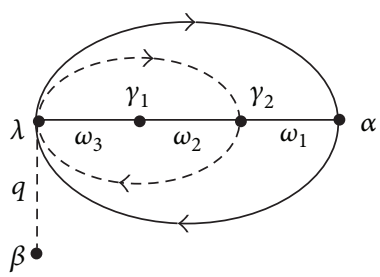

(c)

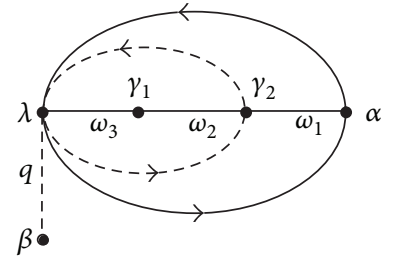

(d)

FigURE 10: Schematic diagrams of (a) $\left|C_{\lambda \alpha}(q)\right|^{2} G_{\gamma_{2} \lambda}^{(-)}\left(\bar{\omega}_{1}\right) P_{+}\left(\gamma_{2}, \lambda\right)$, (b) $\left|C_{\lambda \alpha}(q)\right|^{2} G_{\gamma_{2} \lambda}^{(+)}\left(\bar{\omega}_{1}\right) P_{-}\left(\gamma_{2}, \lambda\right),(\mathrm{c})\left|C_{\beta \lambda}(q)\right|^{2} G_{\lambda \alpha}^{(-)}\left(\bar{\omega}_{123}\right)\left[P_{+}(\lambda, \alpha)-P_{+}\left(\lambda, \gamma_{2}\right)\right]$, and (d) $\left|C_{\beta \lambda}(q)\right|^{2} G_{\lambda \alpha}^{(+)}\left(\bar{\omega}_{123}\right)\left[P_{-}(\lambda, \alpha)-P_{-}\left(\lambda, \gamma_{2}\right)\right]$ in $\Gamma_{\beta \gamma_{1} \gamma_{2} \alpha}^{(24)}\left(\bar{\omega}_{1}, \bar{\omega}_{2}, \bar{\omega}_{3}\right)$.

$$
\begin{aligned}
& -G_{\gamma_{1} \beta}^{(+)}\left(\bar{\omega}_{1}\right) C_{\lambda_{1} \alpha}(q) C_{\lambda_{2} \gamma_{2}}(-q) \delta_{\lambda_{1} \gamma_{1}^{\prime}} \\
& \times\left[b_{q} a_{\gamma_{1}}^{+} a_{\beta}, b_{q}^{+} a_{\lambda_{2}}^{+} a_{\gamma_{2}^{\prime}}\right] \\
& -G_{\gamma_{1} \beta}^{(-)}\left(\bar{\omega}_{1}\right) C_{\lambda_{1} \alpha}(q) C_{\lambda_{2} \gamma_{2}}(-q) \delta_{\lambda_{1} \gamma_{1}^{\prime}} \\
& \times\left[b_{q}^{+} a_{\gamma_{1}}^{+} a_{\beta}, b_{q} a_{\lambda_{2}}^{+} a_{\gamma_{2}^{\prime}}\right] \\
& +G_{\gamma_{1} \beta}^{(+)}\left(\bar{\omega}_{1}\right) C_{\lambda_{1} \alpha}(q) C_{\gamma_{2}^{\prime} \lambda_{2}}(-q) \delta_{\lambda_{1} \gamma_{1}^{\prime}} \\
& \times\left[b_{q} a_{\gamma_{1}}^{+} a_{\beta}, b_{q}^{+} a_{\gamma_{2}}^{+} a_{\lambda_{2}}\right] \\
& +G_{\gamma_{1} \beta}^{(-)}\left(\bar{\omega}_{1}\right) C_{\lambda_{1} \alpha}(q) C_{\gamma_{2}^{\prime} \lambda_{2}}(-q) \delta_{\lambda_{1} \gamma_{1}^{\prime}} \\
& \times\left[b_{q}^{+} a_{\gamma_{1}}^{+} a_{\beta}, b_{q} a_{\gamma_{2}}^{+} a_{\lambda_{2}}\right] \\
& -G_{\alpha \gamma_{1}^{\prime}}^{(+)}\left(\bar{\omega}_{1}\right) C_{\beta \lambda_{1}}(q) C_{\lambda_{2} \gamma_{2}}(-q) \delta_{\lambda_{1} \gamma_{1}} \\
& \times\left[b_{q} a_{\alpha}^{+} a_{\gamma_{1}^{\prime}}, b_{q}^{+} a_{\lambda_{2}}^{+} a_{\gamma_{2}^{\prime}}\right] \\
& -G_{\alpha \gamma_{1}^{\prime}}^{(-)}\left(\bar{\omega}_{1}\right) C_{\beta \lambda_{1}}(q) C_{\lambda_{2} \gamma_{2}}(-q) \delta_{\lambda_{1} \gamma_{1}} \\
& \times\left[b_{q}^{+} a_{\alpha}^{+} a_{\gamma_{1}^{\prime}}, b_{q} a_{\lambda_{2}}^{+} a_{\gamma_{2}^{\prime}}\right] \\
& +G_{\alpha \gamma_{1}^{\prime}}^{(+)}\left(\bar{\omega}_{1}\right) C_{\beta \lambda_{1}}(q) C_{\gamma_{2}^{\prime} \lambda_{2}}(-q) \delta_{\lambda_{1} \gamma_{1}} \\
& \times\left[b_{q} a_{\alpha}^{+} a_{\gamma_{1}^{\prime}}, b_{q}^{+} a_{\gamma_{2}}^{+} a_{\lambda_{2}}\right] \\
& +G_{\alpha \gamma_{1}^{\prime}}^{(-)}\left(\bar{\omega}_{1}\right) C_{\beta \lambda_{1}}(q) C_{\gamma_{2}^{\prime} \lambda_{2}}(-q) \delta_{\lambda_{1} \gamma_{1}} \\
& \times\left[b_{q}^{+} a_{\alpha}^{+} a_{\gamma_{1}^{\prime}}, b_{q} a_{\gamma_{2}}^{+} a_{\lambda_{2}}\right] \\
& +G_{\gamma_{1} \lambda_{1}}^{(+)}\left(\bar{\omega}_{1}\right) C_{\beta \lambda_{1}}(q) C_{\lambda_{2} \gamma_{2}}(-q) \delta_{\alpha \gamma_{1}^{\prime}} \\
& \times\left[b_{q} a_{\gamma_{1}}^{+} a_{\lambda_{1}}, b_{q}^{+} a_{\lambda_{2}}^{+} a_{\gamma_{2}^{\prime}}\right] \\
& +G_{\gamma_{1} \lambda_{1}}^{(-)}\left(\bar{\omega}_{1}\right) C_{\beta \lambda_{1}}(q) C_{\lambda_{2} \gamma_{2}}(-q) \delta_{\alpha \gamma_{1}^{\prime}} \\
& \times\left[b_{q}^{+} a_{\gamma_{1}}^{+} a_{\lambda_{1}}, b_{q} a_{\lambda_{2}}^{+} a_{\gamma_{2}^{\prime}}\right] \\
& -G_{\gamma_{1} \lambda_{1}}^{(+)}\left(\bar{\omega}_{1}\right) C_{\beta \lambda_{1}}(q) C_{\gamma_{2}^{\prime} \lambda_{2}}(-q) \delta_{\alpha \gamma_{1}^{\prime}} \\
& \times\left[b_{q} a_{\gamma_{1}}^{+} a_{\lambda_{1}}, b_{q}^{+} a_{\gamma_{2}}^{+} a_{\lambda_{2}}\right]
\end{aligned}
$$

$$
\begin{aligned}
& -G_{\gamma_{1} \lambda_{1}}^{(-)}\left(\bar{\omega}_{1}\right) C_{\beta \lambda_{1}}(q) C_{\gamma_{2}^{\prime} \lambda_{2}}(-q) \delta_{\alpha \gamma_{1}^{\prime}} \\
& \left.\left.\times\left[b_{q}^{+} a_{\gamma_{1}}^{+} a_{\lambda_{1}}, b_{q} a_{\gamma_{2}}^{+} a_{\lambda_{2}}\right]\right)\right\} \\
& =-\sum_{q, \lambda}\left\{G_{\gamma_{1}^{\prime} \lambda}^{(+)}\left(\bar{\omega}_{1}\right) C_{\lambda \alpha}(q) C_{\gamma_{2}^{\prime} \lambda}(-q)\right. \\
& \times \delta_{\beta \gamma_{1}} \delta_{\gamma_{1}^{\prime} \gamma_{2}} P_{\leftarrow}\left(\lambda, \gamma_{1}^{\prime}\right) \\
& +G_{\gamma_{1}^{\prime \lambda}}^{(-)}\left(\bar{\omega}_{1}\right) C_{\lambda \alpha}(q) C_{\gamma_{2}^{\prime} \lambda}(-q) \\
& \times \delta_{\beta \gamma_{1}} \delta_{\gamma_{1}^{\prime} \gamma_{2}} P_{\rightarrow}\left(\gamma_{1}^{\prime}, \lambda\right) \\
& +G_{\lambda \gamma_{1}}^{(+)}\left(\bar{\omega}_{1}\right) C_{\beta \lambda}(q) C_{\lambda \gamma_{2}}(-q) \\
& \times \delta_{\alpha \gamma_{1}^{\prime}} \delta_{\gamma_{1} \gamma_{2}^{\prime}} P_{\leftarrow}\left(\lambda, \gamma_{1}\right) \\
& -G_{\lambda \gamma_{1}}^{(-)}\left(\bar{\omega}_{1}\right) C_{\beta \lambda}(q) C_{\lambda \gamma_{2}}(-q) \\
& \left.\times \delta_{\alpha \gamma_{1}^{\prime}} \delta_{\gamma_{1} \gamma_{2}^{\prime}} P_{\rightarrow}\left(\lambda, \gamma_{1}\right)\right\} \text {, } \\
& V_{12}\left(\bar{\omega}_{1}, \bar{\omega}_{2}\right) \\
& =\sum_{q} \sum_{\lambda_{1}, \lambda_{2}} T_{R} \\
& \times\left\{\rho _ { \mathrm { eq } } \left(\left[G_{\lambda_{1} \beta}^{(+)}\left(\bar{\omega}_{12}\right) C_{\lambda_{1} \alpha}(q) C_{\lambda_{2} \gamma_{1}}(-q)\right.\right.\right. \\
& \left.\times\left[b_{q} a_{\lambda_{1}}^{+} a_{\beta}, b_{q}^{+} a_{\lambda_{2}}^{+} a_{\gamma_{1}^{\prime}}\right], a_{\gamma_{2}}^{+} a_{\gamma_{2}^{\prime}}\right] \\
& +\left[G_{\lambda_{1} \beta}^{(-)}\left(\bar{\omega}_{12}\right) C_{\lambda_{1} \alpha}(q) C_{\lambda_{2} \gamma_{1}}(-q)\right. \\
& \left.\times\left[b_{q}^{+} a_{\lambda_{1}}^{+} a_{\beta}, b_{q} a_{\lambda_{2}}^{+} a_{\gamma_{1}^{\prime}}\right], a_{\gamma_{2}}^{+} a_{\gamma_{2}^{\prime}}\right] \\
& -\left[G_{\lambda_{1} \beta}^{(+)}\left(\bar{\omega}_{12}\right) C_{\lambda_{1} \alpha}(q) C_{\gamma_{1}^{\prime} \lambda_{2}}(-q)\right. \\
& \left.\times\left[b_{q} a_{\lambda_{1}}^{+} a_{\beta}, b_{q}^{+} a_{\gamma_{1}}^{+} a_{\lambda_{2}}\right], a_{\gamma_{2}}^{+} a_{\gamma_{2}^{\prime}}\right] \\
& \text { - }\left[G_{\lambda_{1} \beta}^{(-)}\left(\bar{\omega}_{12}\right) C_{\lambda_{1} \alpha}(q) C_{\gamma_{1}^{\prime} \lambda_{2}}(-q)\right. \\
& \left.\times\left[b_{q}^{+} a_{\lambda_{1}}^{+} a_{\beta}, b_{q} a_{\gamma_{1}}^{+} a_{\lambda_{2}}\right], a_{\gamma_{2}}^{+} a_{\gamma_{2}^{\prime}}\right]
\end{aligned}
$$




$$
\begin{array}{r}
-\left[G_{\alpha \lambda_{1}}^{(+)}\left(\bar{\omega}_{12}\right) C_{\beta \lambda_{1}}(q) C_{\lambda_{2} \gamma_{1}}(-q)\right. \\
\left.\times\left[b_{q} a_{\alpha}^{+} a_{\lambda_{1}}, b_{q}^{+} a_{\lambda_{2}}^{+} a_{\gamma_{1}^{\prime}}\right], a_{\gamma_{2}}^{+} a_{\gamma_{2}^{\prime}}\right] \\
-\left[G_{\alpha \lambda_{1}}^{(-)}\left(\bar{\omega}_{12}\right) C_{\beta \lambda_{1}}(q) C_{\lambda_{2} \gamma_{1}}(-q)\right. \\
\left.\times\left[b_{q}^{+} a_{\alpha}^{+} a_{\lambda_{1}}, b_{q} a_{\lambda_{2}}^{+} a_{\gamma_{1}^{\prime}}\right], a_{\gamma_{2}}^{+} a_{\gamma_{2}^{\prime}}\right] \\
+\left[G_{\alpha \lambda_{1}}^{(+)}\left(\bar{\omega}_{12}\right) C_{\beta \lambda_{1}}(q) C_{\gamma_{1}^{\prime} \lambda_{2}}(-q)\right. \\
\left.\times\left[b_{q} a_{\alpha}^{+} a_{\lambda_{1}}, b_{q}^{+} a_{\gamma_{1}}^{+} a_{\lambda_{2}}\right], a_{\gamma_{2}}^{+} a_{\gamma_{2}^{\prime}}\right] \\
-\left[G_{\alpha \lambda_{1}}^{(-)}\left(\bar{\omega}_{12}\right) C_{\beta \lambda_{1}}(q) C_{\gamma_{1}^{\prime} \lambda_{2}}(-q)\right. \\
\left.\left.\left.\times\left[b_{q}^{+} a_{\alpha}^{+} a_{\lambda_{1}}, b_{q} a_{\gamma_{1}}^{+} a_{\lambda_{2}}\right], a_{\gamma_{2}}^{+} a_{\gamma_{2}^{\prime}}\right)\right]\right\} \\
=\sum G_{q, \lambda}^{(+)}\left(\bar{\omega}_{12}\right) C_{\lambda \alpha}(q) C_{\gamma_{1}^{\prime} \lambda}(-q) \delta_{\beta \gamma_{2}} \delta_{\gamma_{1} \gamma_{2}^{\prime}} \\
\times\left[P_{\leftarrow}(\beta, \lambda)-P_{\leftarrow}\left(\gamma_{1}, \lambda\right)\right] \\
+G_{\beta \lambda}^{(-)}\left(\bar{\omega}_{12}\right) C_{\lambda \alpha}(q) C_{\gamma_{1}^{\prime} \lambda}(-q) \delta_{\beta \gamma_{2}} \delta_{\gamma_{1} \gamma_{2}^{\prime}} \\
\times\left[P \rightarrow\left(\gamma_{1}, \lambda\right)-P \rightarrow(\beta, \lambda)\right] \\
\left.\times\left[P_{\rightarrow}\left(\lambda, \gamma_{1}^{\prime}\right)-P_{\rightarrow}(\lambda, \alpha)\right]\right\} \\
\times G_{\lambda \alpha}^{(+)}\left(\bar{\omega}_{12}\right) C_{\beta \lambda}(q) C_{\lambda \gamma_{1}}(-q) \delta_{\alpha \gamma_{2}^{\prime}} \delta_{\gamma_{1}^{\prime} \gamma_{2}} \\
\times\left[P_{\leftarrow}(\lambda, \alpha)-P_{\rightarrow}\left(\lambda, \gamma_{1}^{\prime}\right)\right] \\
-G_{\lambda \alpha}^{(-)}\left(\bar{\omega}_{12}\right) C_{\beta \lambda}(q) C_{\lambda \gamma_{1}}(-q) \delta_{\alpha \gamma_{2}^{\prime}} \delta_{\gamma_{1}^{\prime} \gamma_{2}} \\
\times\left[P_{1}\right)
\end{array}
$$

$$
V_{21}\left(\bar{\omega}_{1}\right)
$$$$
=\sum_{q} \sum_{\lambda_{1}, \lambda_{2}} T_{R}\left\{\rho _ { \mathrm { eq } } \left[G_{d}\left(\bar{\omega}_{1}\right)\right.\right.
$$

$$
\begin{aligned}
& \times\left\{C_{\lambda_{1} \alpha}(q)\left(b_{q}+b_{q}^{+}\right) \delta_{\beta \gamma_{1}}\right. \\
& \times\left(a_{\lambda_{1}}^{+} a_{\gamma_{2}^{\prime}} \delta_{\gamma_{1}^{\prime} \gamma_{2}}-a_{\gamma_{2}}^{+} a_{\gamma_{1}^{\prime}} \delta_{\lambda_{1} \gamma_{2}^{\prime}}\right) \\
&-C_{\lambda_{1} \alpha}(q)\left(b_{q}+b_{q}^{+}\right) \delta_{\lambda_{1} \gamma_{1}^{\prime}} \\
& \times\left(a_{\gamma_{1}}^{+} a_{\gamma_{2}^{\prime}} \delta_{\beta \gamma_{2}}-a_{\gamma_{2}}^{+} a_{\beta} \delta_{\gamma_{1} \gamma_{2}^{\prime}}\right) \\
&-C_{\beta \lambda_{1}}(q)\left(b_{q}+b_{q}^{+}\right) \delta_{\lambda_{1} \gamma_{1}} \\
& \times\left(a_{\alpha}^{+} a_{\gamma_{2}^{\prime}} \delta_{\gamma_{1}^{\prime} \gamma_{2}}-a_{\gamma_{2}}^{+} a_{\gamma_{1}^{\prime}} \delta_{\alpha \gamma_{2}^{\prime}}\right) \\
&+C_{\beta \lambda_{1}}(q)\left(b_{q}+b_{q}^{+}\right) \delta_{\alpha \gamma_{1}^{\prime}} \\
&\left.\times\left(a_{\gamma_{1}}^{+} a_{\gamma_{2}^{\prime}} \delta_{\lambda_{1} \gamma_{2}}-a_{\gamma_{2}}^{+} a_{\lambda_{1}} \delta_{\gamma_{1} \gamma_{2}^{\prime}}\right)\right\}, \\
&\left(b_{q}+\right.\left.b_{q}^{+}\right) \\
&\left.\left.\times\left\{C_{\lambda_{2} \gamma_{3}}(-q) a_{\lambda_{2}}^{+} a_{\gamma_{3}^{\prime}}-C_{\gamma_{3}^{\prime} \lambda_{2}}\left(q_{2}\right) a_{\gamma_{3}}^{+} a_{\lambda_{2}}\right\}\right]\right\}
\end{aligned}
$$

$$
\begin{aligned}
=-\sum_{q, \lambda}\{ & G_{\gamma_{2}^{\prime} \lambda}^{(+)}\left(\bar{\omega}_{1}\right) C_{\lambda \alpha}(q) C_{\gamma_{3}^{\prime} \lambda}(-q) \delta_{\beta \gamma_{1}} \delta_{\gamma_{1}^{\prime} \gamma_{2}} \delta_{\gamma_{2}^{\prime} \gamma_{3}} \\
& \times\left[P_{\leftarrow}\left(\gamma_{2}^{\prime}, \lambda\right)\right]+G_{\gamma_{2}^{\prime} \lambda}^{(-)}\left(\bar{\omega}_{1}\right) C_{\lambda \alpha}(q) C_{\gamma_{3}^{\prime} \lambda}(-q) \\
& \times \delta_{\beta \gamma_{1}} \delta_{\gamma_{1}^{\prime} \gamma_{2}} \delta_{\gamma_{2}^{\prime} \gamma_{3}}\left[P_{\rightarrow}\left(\gamma_{2}^{\prime}, \lambda\right)\right] \\
& -G_{\lambda \gamma_{2}}^{(+)}\left(\bar{\omega}_{1}\right) C_{\beta \lambda}(q) C_{\lambda \gamma_{3}}(-q) \\
& \times \delta_{\alpha \gamma_{1}^{\prime}} \delta_{\gamma_{1} \gamma_{2}^{\prime}} \delta_{\gamma_{2} \gamma_{3}^{\prime}}\left[P_{\leftarrow}\left(\lambda, \gamma_{2}\right)\right] \\
& +G_{\lambda \gamma_{2}}^{(-)}\left(\bar{\omega}_{1}\right) C_{\beta \lambda}(q) C_{\lambda \gamma_{3}}(-q) \\
& \left.\times \delta_{\alpha \gamma_{1}^{\prime}} \delta_{\gamma_{1} \gamma_{2}^{\prime}} \delta_{\gamma_{2} \gamma_{3}^{\prime}}\left[P_{\rightarrow}\left(\lambda, \gamma_{2}\right)\right]\right\}
\end{aligned}
$$$$
V_{22}\left(\bar{\omega}_{1}, \bar{\omega}_{2}\right)
$$$$
=\sum_{q} \sum_{\lambda_{1}, \lambda_{2}} T_{R}
$$$$
\times\left\{\rho _ { \mathrm { eq } } \left(\left[G_{\lambda_{1} \gamma_{1}^{\prime}}^{(+)}\left(\bar{\omega}_{12}\right) C_{\lambda_{1} \alpha}(q) C_{\lambda_{2} \gamma_{2}}(-q) \delta_{\beta \gamma_{1}}\right.\right.\right.
$$$$
\left.\times\left[b_{q} a_{\lambda_{1}}^{+} a_{\gamma_{1}^{\prime}}, b_{q}^{+} a_{\lambda_{2}}^{+} a_{\gamma_{2}^{\prime}}\right], a_{\gamma_{3}}^{+} a_{\gamma_{3}^{\prime}}\right]
$$$$
-\left[G_{\lambda_{1} \gamma_{1}^{\prime}}^{(+)}\left(\bar{\omega}_{12}\right) C_{\lambda_{1} \alpha}(q) C_{\gamma_{2}^{\prime} \lambda_{2}}(-q) \delta_{\beta \gamma_{1}}\right.
$$$$
\left.\times\left[b_{q} a_{\lambda_{1}}^{+} a_{\gamma_{1}^{\prime}}, b_{q}^{+} a_{\gamma_{2}}^{+} a_{\lambda_{2}}\right], a_{\gamma_{3}}^{+} a_{\gamma_{3}^{\prime}}\right]
$$$$
+\left[G_{\lambda_{1} \gamma_{1}^{\prime}}^{(-)}\left(\bar{\omega}_{12}\right) C_{\lambda_{1} \alpha}(q) C_{\lambda_{2} \gamma_{2}}(-q) \delta_{\beta \gamma_{1}}\right.
$$$$
\left.\times\left[b_{q}^{+} a_{\lambda_{1}}^{+} a_{\gamma_{1}^{\prime}}, b_{q} a_{\lambda_{2}}^{+} a_{\gamma_{2}^{\prime}}\right], a_{\gamma_{3}}^{+} a_{\gamma_{3}^{\prime}}\right]
$$$$
-\left[G_{\lambda_{1} \gamma_{1}^{\prime}}^{(-)}\left(\bar{\omega}_{12}\right) C_{\lambda_{1} \alpha}(q) C_{\gamma_{2}^{\prime} \lambda_{2}}(-q) \delta_{\beta \gamma_{1}}\right.
$$$$
\left.\times\left[b_{q}^{+} a_{\lambda_{1}}^{+} a_{\gamma_{1}^{\prime}}, b_{q} a_{\gamma_{2}}^{+} a_{\lambda_{2}}\right], a_{\gamma_{3}}^{+} a_{\gamma_{3}^{\prime}}\right]
$$$$
+\left[G_{\gamma_{1} \lambda_{1}}^{(+)}\left(\bar{\omega}_{12}\right) C_{\beta \lambda_{1}}(q) C_{\lambda_{2} \gamma_{2}}(-q) \delta_{\alpha \gamma_{1}^{\prime}}\right.
$$$$
\left.\times\left[b_{q} a_{\gamma_{1}}^{+} a_{\lambda_{1}}, b_{q}^{+} a_{\lambda_{2}}^{+} a_{\gamma_{2}^{\prime}}\right], a_{\gamma_{3}}^{+} a_{\gamma_{3}^{\prime}}\right]
$$$$
\text { - }\left[G_{\gamma_{1} \lambda_{1}}^{(+)}\left(\bar{\omega}_{12}\right) C_{\beta \lambda_{1}}(q) C_{\gamma_{2}^{\prime} \lambda_{2}}(-q) \delta_{\alpha \gamma_{1}^{\prime}}\right.
$$$$
\left.\times\left[b_{q} a_{\gamma_{1}}^{+} a_{\lambda_{1}}, b_{q}^{+} a_{\gamma_{2}}^{+} a_{\lambda_{2}}\right], a_{\gamma_{3}}^{+} a_{\gamma_{3}^{\prime}}\right]
$$$$
+\left[G_{\gamma_{1} \lambda_{1}}^{(-)}\left(\bar{\omega}_{12}\right) C_{\beta \lambda_{1}}(q) C_{\lambda_{2} \gamma_{2}}(-q) \delta_{\alpha \gamma_{1}^{\prime}}\right.
$$$$
\left.\times\left[b_{q}^{+} a_{\gamma_{1}}^{+} a_{\lambda_{1}}, b_{q} a_{\lambda_{2}}^{+} a_{\gamma_{2}^{\prime}}\right], a_{\gamma_{3}}^{+} a_{\gamma_{3}^{\prime}}\right]
$$$$
-\left[G_{\gamma_{1} \lambda_{1}}^{(-)}\left(\bar{\omega}_{12}\right) C_{\beta \lambda_{1}}(q) C_{\gamma_{2}^{\prime} \lambda_{2}}(-q) \delta_{\alpha \gamma_{1}^{\prime}}\right.
$$$$
\left.\left.\left.\times\left[b_{q}^{+} a_{\gamma_{1}}^{+} a_{\lambda_{1}}, b_{q} a_{\gamma_{2}}^{+} a_{\lambda_{2}}\right], a_{\gamma_{3}}^{+} a_{\gamma_{3}^{\prime}}\right]\right)\right\}
$$ 
$=\sum_{q, \lambda}\left\{G_{\gamma_{1}^{\prime} \lambda}^{(+)}\left(\bar{\omega}_{12}\right) C_{\lambda \alpha}(q) C_{\gamma_{2}^{\prime} \lambda}(-q)\right.$

$$
\times \delta_{\beta \gamma_{1}} \delta_{\gamma_{3}^{\prime} \gamma_{2}} \delta_{\gamma_{1}^{\prime} \gamma_{3}}\left[P_{\leftarrow}\left(\gamma_{1}^{\prime}, \lambda\right)-P_{\leftarrow}\left(\gamma_{2}, \lambda\right)\right]
$$$$
+G_{\gamma_{1}^{\prime} \lambda}^{(-)}\left(\bar{\omega}_{12}\right) C_{\lambda \alpha}(q) C_{\gamma_{2}^{\prime} \lambda}(-q)
$$$$
\times \delta_{\beta \gamma_{1}} \delta_{\gamma_{3}^{\prime} \gamma_{2}} \delta_{\gamma_{1}^{\prime} \gamma_{3}}\left[P_{\rightarrow}\left(\gamma_{2}, \lambda\right)-P_{\rightarrow}\left(\gamma_{1}^{\prime}, \lambda\right)\right]
$$$$
+G_{\lambda \gamma_{1}}^{(+)}\left(\bar{\omega}_{12}\right) C_{\beta \lambda}(q) C_{\lambda \gamma_{2}}(-q)
$$$$
\times \delta_{\alpha \gamma_{1}^{\prime}} \delta_{\gamma_{3} \gamma_{2}^{\prime}} \delta_{\gamma_{1} \gamma_{3}^{\prime}}\left[P_{\leftarrow}\left(\lambda, \gamma_{1}\right)-P_{\leftarrow}\left(\lambda, \gamma_{2}^{\prime}\right)\right]
$$$$
+G_{\lambda \gamma_{1}}^{(-)}\left(\bar{\omega}_{12}\right) C_{\beta \lambda}(q) C_{\lambda \gamma_{2}}(-q)
$$$$
\left.\times \delta_{\alpha \gamma_{1}^{\prime}} \delta_{\gamma_{3} \gamma_{2}^{\prime}} \delta_{\gamma_{1} \gamma_{3}^{\prime}}\left[P_{\rightarrow}\left(\lambda, \gamma_{2}^{\prime}\right)-P_{\rightarrow}\left(\lambda, \gamma_{1}\right)\right]\right\} \text {, }
$$

$V_{24}\left(\bar{\omega}_{1}, \bar{\omega}_{2}, \bar{\omega}_{3}\right)$

$$
\begin{aligned}
& =\sum_{q} \sum_{\lambda_{1}, \lambda_{2}} T_{R} \\
& \times\left\{\rho _ { \mathrm { eq } } \left(\left[\left[G_{d}\left(\bar{\omega}_{12}\right) G_{\lambda_{1} \beta}^{(+)}\left(\bar{\omega}_{123}\right) C_{\lambda_{1} \alpha}(q) C_{\lambda_{2} \gamma_{1}}(-q)\right.\right.\right.\right. \\
& \left.\left.\times\left[b_{q} a_{\lambda_{1}}^{+} a_{\beta}, b_{q}^{+} a_{\lambda_{2}}^{+} a_{\gamma_{1}^{\prime}}\right], a_{\gamma_{2}}^{+} a_{\gamma_{2}^{\prime}}\right], a_{\gamma_{3}}^{+} a_{\gamma_{3}^{\prime}}\right] \\
& +\left[\left[G_{d}\left(\bar{\omega}_{12}\right) G_{\lambda_{1} \beta}^{(-)}\left(\bar{\omega}_{123}\right) C_{\lambda_{1} \alpha}(q) C_{\lambda_{2} \gamma_{1}}(-q)\right.\right. \\
& \left.\left.\times\left[b_{q}^{+} a_{\lambda_{1}}^{+} a_{\beta}, b_{q} a_{\lambda_{2}}^{+} a_{\gamma_{1}^{\prime}}\right], a_{\gamma_{2}}^{+} a_{\gamma_{2}^{\prime}}\right], a_{\gamma_{3}}^{+} a_{\gamma_{3}^{\prime}}\right] \\
& \text { - }\left[\left[G_{d}\left(\bar{\omega}_{12}\right) G_{\lambda_{1} \beta}^{(+)}\left(\bar{\omega}_{123}\right) C_{\lambda_{1} \alpha}(q) C_{\gamma_{1}^{\prime} \lambda_{2}}(-q)\right.\right. \\
& \left.\left.\times\left[b_{q} a_{\lambda_{1}}^{+} a_{\beta}, b_{q}^{+} a_{\gamma_{1}}^{+} a_{\lambda_{2}}\right], a_{\gamma_{2}}^{+} a_{\gamma_{2}^{\prime}}\right], a_{\gamma_{3}}^{+} a_{\gamma_{3}^{\prime}}\right] \\
& -\left[\left[G_{d}\left(\bar{\omega}_{12}\right) G_{\lambda_{1} \beta}^{(-)}\left(\bar{\omega}_{123}\right) C_{\lambda_{1} \alpha}(q) C_{\gamma_{1}^{\prime} \lambda_{2}}(-q)\right.\right. \\
& \left.\left.\times\left[b_{q}^{+} a_{\lambda_{1}}^{+} a_{\beta}, b_{q} a_{\gamma_{1}}^{+} a_{\lambda_{2}}\right], a_{\gamma_{2}}^{+} a_{\gamma_{2}^{\prime}}\right], a_{\gamma_{3}}^{+} a_{\gamma_{3}^{\prime}}\right] \\
& \text { - }\left[\left[G_{d}\left(\bar{\omega}_{12}\right) G_{\alpha \lambda_{1}}^{(+)}\left(\bar{\omega}_{123}\right) C_{\beta \lambda_{1}}(q) C_{\lambda_{2} \gamma_{1}}(-q)\right.\right. \\
& \left.\left.\times\left[b_{q} a_{\alpha}^{+} a_{\lambda_{1}}, b_{q}^{+} a_{\lambda_{2}}^{+} a_{\gamma_{1}^{\prime}}\right], a_{\gamma_{2}}^{+} a_{\gamma_{2}^{\prime}}\right], a_{\gamma_{3}}^{+} a_{\gamma_{3}^{\prime}}\right] \\
& \text { - }\left[\left[G_{d}\left(\bar{\omega}_{12}\right) G_{\alpha \lambda_{1}}^{(-)}\left(\bar{\omega}_{123}\right) C_{\beta \lambda_{1}}(q) C_{\lambda_{2} \gamma_{1}}(-q)\right.\right. \\
& \left.\left.\times\left[b_{q}^{+} a_{\alpha}^{+} a_{\lambda_{1}}, b_{q} a_{\lambda_{2}}^{+} a_{\gamma_{1}^{\prime}}\right], a_{\gamma_{2}}^{+} a_{\gamma_{2}^{\prime}}\right], a_{\gamma_{3}}^{+} a_{\gamma_{3}^{\prime}}\right] \\
& +\left[\left[G_{d}\left(\bar{\omega}_{12}\right) G_{\alpha \lambda_{1}}^{(+)}\left(\bar{\omega}_{123}\right) C_{\beta \lambda_{1}}(q) C_{\gamma_{1}^{\prime} \lambda_{2}}(-q)\right.\right. \\
& \left.\left.\times\left[b_{q} a_{\alpha}^{+} a_{\lambda_{1}}, b_{q}^{+} a_{\gamma_{1}}^{+} a_{\lambda_{2}}\right], a_{\gamma_{2}}^{+} a_{\gamma_{2}^{\prime}}\right], a_{\gamma_{3}}^{+} a_{\gamma_{3}^{\prime}}\right] \\
& +\left[\left[G_{d}\left(\bar{\omega}_{12}\right) G_{\alpha \lambda_{1}}^{(-)}\left(\bar{\omega}_{123}\right) C_{\beta \lambda_{1}}(q) C_{\gamma_{1}^{\prime} \lambda_{2}}(-q)\right.\right. \\
& \left.\left.\left.\left.\times\left[b_{q}^{+} a_{\alpha}^{+} a_{\lambda_{1}}, b_{q} a_{\gamma_{1}}^{+} a_{\lambda_{2}}\right], a_{\gamma_{2}}^{+} a_{\gamma_{2}^{\prime}}\right], a_{\gamma_{3}}^{+} a_{\gamma_{3}^{\prime}}\right]\right)\right\}
\end{aligned}
$$

$=\sum_{q, \lambda}\left\{\frac{C_{\lambda \alpha}(q) C_{\gamma_{1}^{\prime} \lambda}(-q)}{\hbar \bar{\omega}_{12}-E_{\gamma_{1} \beta}} G_{\beta \lambda}^{(+)}\left(\bar{\omega}_{123}\right)\right.$

$\times \delta_{\gamma_{1} \gamma_{2}^{\prime}} \delta_{\gamma_{3}^{\prime} \gamma_{2}} \delta_{\beta \gamma_{3}}\left[P_{\leftarrow}\left(\gamma_{2}, \lambda\right)-P_{\leftarrow}(\beta, \lambda)\right]$

$-\frac{C_{\lambda \alpha}(q) C_{\gamma_{1}^{\prime} \lambda}(-q)}{\hbar \bar{\omega}_{12}-E_{\gamma_{1} \beta}} G_{\beta \lambda}^{(+)}\left(\bar{\omega}_{123}\right)$

$\times \delta_{\beta \gamma_{2}} \delta_{\gamma_{3}^{\prime} \gamma_{1}} \delta_{\gamma_{2}^{\prime} \gamma_{3}}\left[P_{\leftarrow}\left(\gamma_{1}, \lambda\right)-P_{\leftarrow}\left(\gamma_{2}^{\prime}, \lambda\right)\right]$

$+\frac{C_{\lambda \alpha}(q) C_{\gamma_{1}^{\prime} \lambda}(-q)}{\hbar \bar{\omega}_{12}-E_{\gamma_{1} \beta}} G_{\beta \lambda}^{(-)}\left(\bar{\omega}_{123}\right)$

$\times \delta_{\beta \gamma_{2}} \delta_{\gamma_{3}^{\prime} \gamma_{1}} \delta_{\gamma_{2}^{\prime} \gamma_{3}}\left[P_{\rightarrow}\left(\gamma_{1}, \lambda\right)-P_{\rightarrow}\left(\gamma_{2}^{\prime}, \lambda\right)\right]$

$-\frac{C_{\lambda \alpha}(q) C_{\gamma_{1}^{\prime} \lambda}(-q)}{\hbar \bar{\omega}_{12}-E_{\gamma_{1} \beta}} G_{\beta \lambda}^{(-)}\left(\bar{\omega}_{123}\right)$

$\times \delta_{\gamma_{1} \gamma_{2}^{\prime}} \delta_{\gamma_{3} \beta} \delta_{\gamma_{2} \gamma_{3}^{\prime}}\left[P_{\rightarrow}\left(\gamma_{2}, \lambda\right)-P_{\rightarrow}(\beta, \lambda)\right]$

$-\frac{C_{\beta \lambda}(q) C_{\lambda \gamma_{1}}(-q)}{\hbar \bar{\omega}_{12}-E_{\beta \gamma_{1}^{\prime}}} G_{\lambda \alpha}^{(+)}\left(\bar{\omega}_{123}\right)$

$\times \delta_{\gamma_{1}^{\prime} \gamma_{2}} \delta_{\gamma_{3} \gamma_{2}^{\prime}} \delta_{\alpha \gamma_{3}^{\prime}}\left[P_{\leftarrow}(\lambda, \alpha)-P_{\leftarrow}\left(\lambda, \gamma_{2}^{\prime}\right)\right]$

$+\frac{C_{\beta \lambda}(q) C_{\lambda \gamma_{1}}(-q)}{\hbar \bar{\omega}_{12}-E_{\alpha \gamma_{1}^{\prime}}} G_{\lambda \alpha}^{(+)}\left(\bar{\omega}_{123}\right)$

$\times \delta_{\alpha \gamma_{2}^{\prime}} \delta_{\gamma_{3} \gamma_{1}^{\prime}} \delta_{\gamma_{2} \gamma_{3}^{\prime}}\left[P_{\leftarrow}\left(\lambda, \gamma_{2}\right)-P_{\leftarrow}\left(\lambda, \gamma_{1}^{\prime}\right)\right]$

$+\frac{C_{\beta \lambda}(q) C_{\lambda \gamma_{1}}(-q)}{\hbar \bar{\omega}_{12}-E_{\alpha \gamma_{1}^{\prime}}} G_{\lambda \alpha}^{(-)}\left(\bar{\omega}_{123}\right)$

$\times \delta_{\alpha \gamma_{2}^{\prime}} \delta_{\gamma_{3} \gamma_{1}^{\prime}} \delta_{\gamma_{2} \gamma_{3}^{\prime}}\left[P_{\rightarrow}\left(\lambda, \gamma_{1}^{\prime}\right)-P_{\rightarrow}\left(\lambda, \gamma_{2}\right)\right]$

$-\frac{C_{\beta \lambda}(q) C_{\lambda \gamma_{1}}(-q)}{\hbar \bar{\omega}_{12}-E_{\alpha \gamma_{1}^{\prime}}} G_{\lambda \alpha}^{(-)}\left(\bar{\omega}_{123}\right)$

$\left.\times \delta_{\gamma_{1}^{\prime} \gamma_{2}} \delta_{\gamma_{3}^{\prime} \alpha} \delta_{\gamma_{2}^{\prime} \gamma_{3}}\left[P_{\rightarrow}\left(\lambda, \gamma_{2}^{\prime}\right)-P_{\rightarrow}(\lambda, \alpha)\right]\right\}$.

\section{Conflict of Interests}

The authors declare that there is no conflict of interests regarding the publication of this paper.

\section{References}

[1] R. Kubo, "Statistical-mechanical theory of irreversible processes. I: general theory and simple applications to magnetic and conduction problems," Journal of the Physical Society of Japan, vol. 12, no. 6, pp. 570-586, 1957.

[2] R. Zwanzig, "Ensemble method in the theory of irreversibility," The Journal of Chemical Physics, vol. 33, no. 5, pp. 1338-1341, 1960. 
[3] H. Mori, "A continued-fraction representation of the timevorrelation functions," Progress of Theoretical Physics, vol. 34, no. 3, pp. 399-416, 1965.

[4] A. Kawabata, "Theory of cyclotron resonance line width," Journal of the Physical Society of Japan, vol. 23, no. 5, pp. 9991006, 1967.

[5] P. N. Argyres and J. L. Sigel, "Projection techniques in the theory of electrical resistivity," Physical Review Letters, vol. 31, no. 23, pp. 1397-1400, 1973.

[6] S. D. Choi and O. H. Chung, "Theory of cyclotron resonance lineshape for electron-phonon systems in two coupling schemes," Solid State Communications, vol. 46, no. 9, pp. 717720, 1983.

[7] S. N. Yi, O. H. Chung, and S. D. Choi, "Theory of cyclotron resonance lineshape revisited," Progress of Theoretical Physics, vol. 77, no. 4, pp. 891-898, 1987.

[8] J. Y. Ryu and S. D. Choi, "Quantum-statistical theory of highfield transport phenomena," Physical Review B, vol. 44, no. 20, pp. 11328-11338, 1991.

[9] J. Y. Sug and S. D. Choi, "Quantum transport theory based on the equilibrium density projection technique," Physical Review E, vol. 55, no. 1, pp. 314-321, 1997.

[10] S. Badjou and P. N. Argyres, "Theory of cyclotron resonance in an electron-phonon system," Physical Review B, vol. 35, no. 12, pp. 5964-5968, 1987.

[11] Y. J. Cho and S. D. Choi, "Theory of cyclotron-resonance line shapes based on the isolation-projection technique," Physical Review B, vol. 47, no. 15, pp. 9273-9278, 1993.

[12] N. L. Kang, Y. J. Cho, and S. D. Choi, "A many-body theory of quantum-limit cyclotron transition line-shapes in electronphonon systems based on projection technique," Progress of Theoretical Physics, vol. 96, no. 2, pp. 307-316, 1996.

[13] H. J. Lee, N. L. Kang, J. Y. Sug, and S. D. Choi, "Calculation of the nonlinear optical conductivity by a quantum-statistical method," Physical Review B, vol. 65, no. 19, Article ID 195113, 2002.

[14] N. L. Kang and S. D. Choi, "Derivation of nonlinear optical conductivity by using a reduction identity and a state-dependent projection method," Journal of Physics A, vol. 43, no. 16, Article ID 165203, 2010.

[15] N. L. Kang, Y. J. Lee, and S. D. Choi, "Generalization of the nonlinear optical conductivity formalism by using a projectionreduction method," Journal of the Korean Physical Society, vol. 58, no. 3, pp. 538-544, 2011.

[16] N. L. Kang and S. D. Choi, "Validity of nth-order conductivity by the general projection-reduction method," Progress of Theoretical Physics, vol. 125, no. 5, pp. 1011-1019, 2011.

[17] N. L. Kang and S. D. Choi, "Derivation of second-order nonlinear optical conductivity by the projection diagram method," AIP Advances, vol. 2, Article ID 012161, 2012.

[18] P. A. Franken, A. E. Hill, C. W. Peters, and G. Weinreich, "Generation of optical harmonics," Physical Review Letters, vol. 7, no. 4, pp. 118-119, 1961.

[19] J. A. Armstrong, N. Bloembergen, J. Ducuing, and P. S. Pershan, "Interactions between light waves in a nonlinear dielectric," Physical Review, vol. 127, no. 6, pp. 1918-1939, 1962.

[20] N. Bloembergen and Y. R. Shen, "Quantum-theoretical comparison of nonlinear susceptibilities in parametric media, lasers, and Raman lasers," Physical Review, vol.133, no. 1, pp. A37-A49, 1964.
[21] L. V. Keldysh, "Ionization in the Field of a strong electromagnetic wave," Soviet Physics JETP, vol. 20, no. 5, pp. 1307-1314, 1965.

[22] Y. R. Shen, The Principles of Nonlinear Optics, chapter 2, Willey, New York, USA, 1984.

[23] B. S. Wherrett, "Scaling rules for multiphoton interband absorption in semiconductors," Journal of the Optical Society of America B, vol. 1, no. 1, pp. 67-72, 1984.

[24] M. Sheik-Bahae, D. C. Hutchings, D. J. Hagan, and E. W. Van Stryland, "Dispersion of bound electronic nonlinear refraction in solids," IEEE Journal of Quantum Electronics, vol. 27, no. 6, pp. 1296-1309, 1991.

[25] W. S. Fann, R. Storz, H. W. K. Tom, and J. Bokor, "Electron thermalization in gold," Physical Review B, vol. 46, no. 20, pp. 13592-13595, 1992.

[26] C.-K. Sun, F. Vallée, L. H. Acioli, E. P. Ippen, and J. G. Fujimoto, "Femtosecond-tunable measurement of electron thermalization in gold," Physical Review B, vol. 50, no. 20, pp. 15337-15348, 1994.

[27] R. H. M. Groeneveld, R. Sprik, and A. Lagendijk, "Femtosecond spectroscopy of electron-electron and electron-phonon energy relaxation in Ag and Au," Physical Review B, vol. 51, no. 17, pp. 11433-11445, 1995.

[28] A. Suzuki and M. Ashikawa, "Quantum-statistical theory of nonlinear optical conductivity for an electron-phonon system," Physical Review E, vol. 58, no. 4, pp. 4307-4320, 1998.

[29] N. Del Fatti, C. Voisin, M. Achenmann, S. Tzortzakis, D. Christofilos, and F. Vallée, "Nonequilibrium electron dynamics in noble metals," Physical Review B, vol. 61, no. 24, pp. 1695616966, 2000.

[30] Y. Hamanaka, J. Kuwabata, I. Tanahashi, S. Omi, and A. Nakamura, "Ultrafast electron relaxation via breathing vibration of gold nanocrystals embedded in a dielectric medium," Physical Review B, vol. 63, no. 10, Article ID 104302, 2001.

[31] A. Zarifi, C. Fisker, and T. G. Pedersen, "Theoretical study of quadratic electro-optic effect in semiconducting zigzag carbon nanotubes," Physical Review B, vol. 76, no. 4, Article ID 045403, 2007.

[32] N. Olivier and E. Beaurepaire, "Third-harmonic generation microscopy with focus-engineered beams: a numerical study," Optics Express, vol. 16, no. 19, pp. 14703-14708, 2008.

[33] F. Munhoz, H. Rigneault, and S. Brasselet, "High order symmetry structural properties of vibrational resonances using multiple-field polarization coherent anti-stokes Raman spectroscopy microscopy," Physical Review Letters, vol. 105, no. 12, Article ID 123903, 2010.

[34] C.-C. Wu and J. Tsai, "Coupling mechanisms of acoustic second-harmonic generation in piezoelectric semiconductor," Journal of Physics C, vol. 20, no. 10, pp. 1527-1533, 1987.

[35] Y.-M. Chang and N.-A. Chang, "Observation of coherent optical phonons in $\mathrm{GaAs} / \mathrm{Al}_{x} \mathrm{Ga}_{1-x}$ As single heterojunctions," Journal of Applied Physics, vol. 93, no. 4, pp. 2015-2018, 2003.

[36] D. H. Son, J. S. Wittenberg, U. Banin, and A. P. Alivisatos, "Second harmonic generation and confined acoustic phonons in highly excited semiconductor nanocrystals," Journal of Physical Chemistry B, vol. 110, no. 40, pp. 19884-19890, 2006.

[37] R. P. Feynman, "Space-time approach to quantum electrodynamics," Physical Review, vol. 76, no. 6, pp. 769-789, 1949.

[38] A. L. Fetter and J. D. Walecka, Quantum Theory of Many Particle Systems, McGraw Hill,, New York, NY, USA, 1971. 
[39] R. D. Mattuch, A Guide to Feynman Diagrams in the Many Body Problem, McGraw Hill, New York, NY, USA, 2nd edition, 1976.

[40] G. D. Mahan, Many Particle Physics, chapter 3, Plenum, New York, NY, USA, 2000.

[41] F. Giustino, M. L. Cohen, and S. G. Louie, "Small phonon contribution to the photoemission kink in the copper oxide superconductors," Nature, vol. 452, no. 7190, pp. 975-978, 2008.

[42] A. R. Wright and C. Zhang, "Dynamic conductivity of graphene with electron-LO-phonon interaction," Physical Review B, vol. 81, no. 16, Article ID 165413, 2010. 

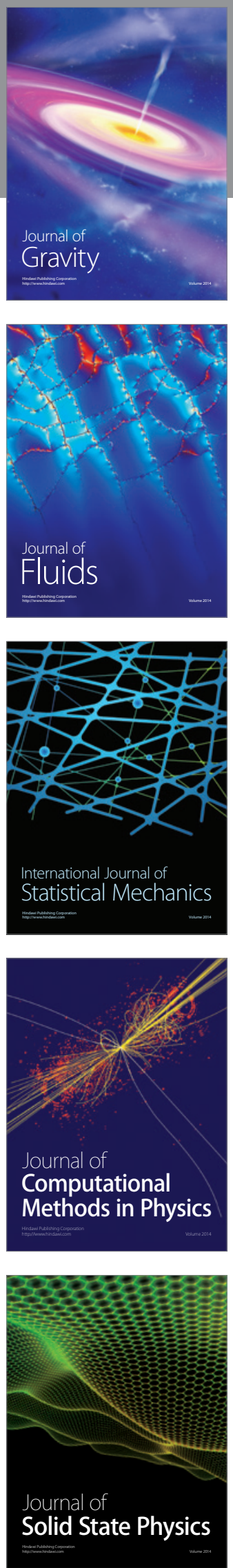

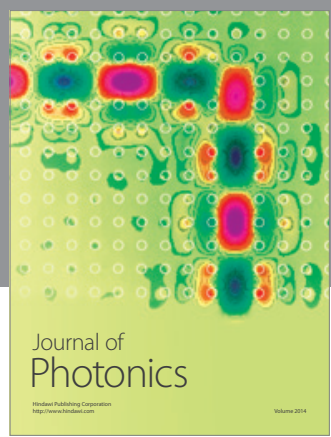

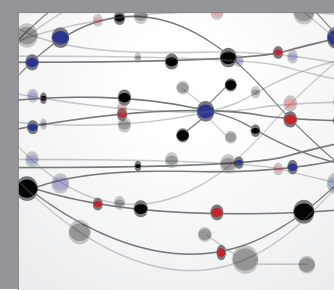

The Scientific World Journal

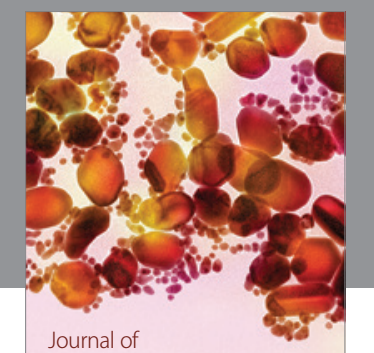

Soft Matter
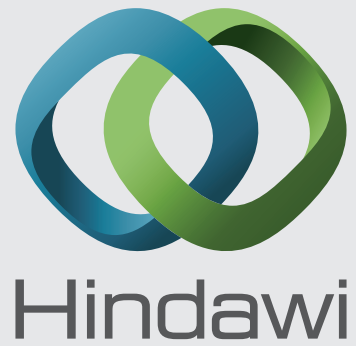

Submit your manuscripts at

http://www.hindawi.com
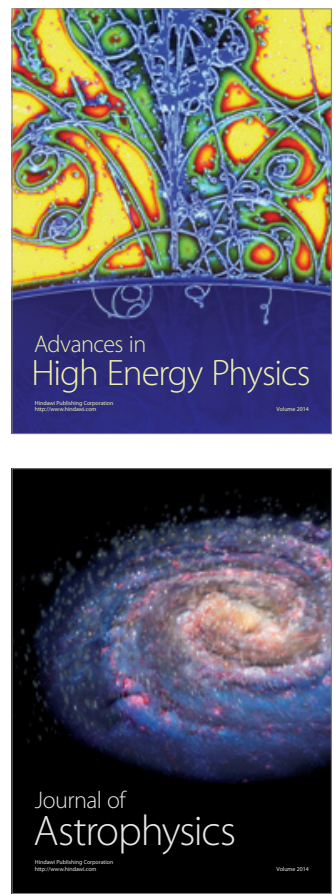
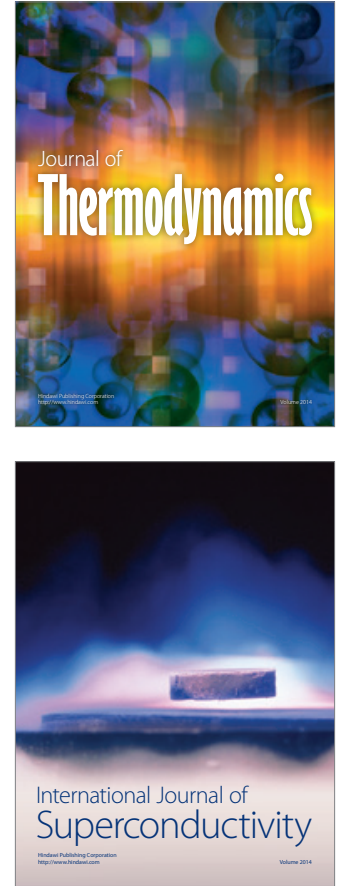
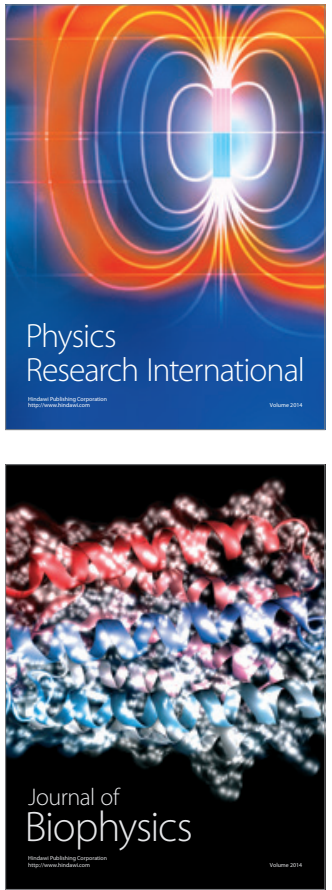
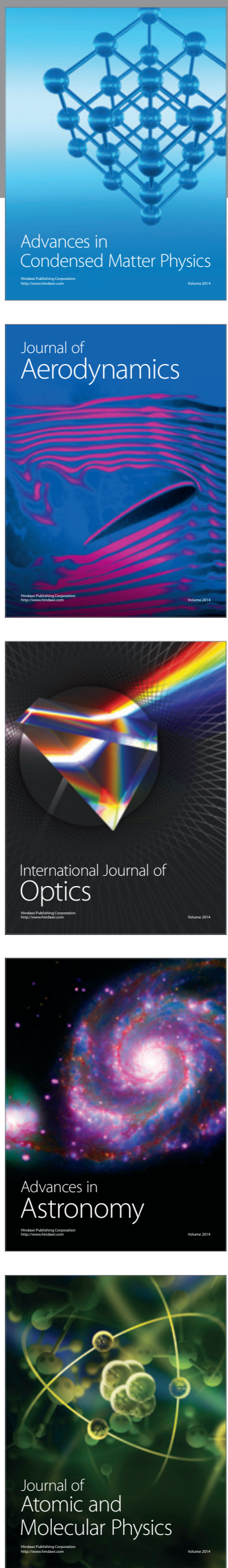